\title{
WestVirginiaUniversity
}

THE RESEARCH REPOSITORY @ WVU

Graduate Theses, Dissertations, and Problem Reports

2005

\section{Username and password verification through keystroke dynamics}

Nick Bartlow

West Virginia University

Follow this and additional works at: https://researchrepository.wvu.edu/etd

\section{Recommended Citation}

Bartlow, Nick, "Username and password verification through keystroke dynamics" (2005). Graduate Theses, Dissertations, and Problem Reports. 1576.

https://researchrepository.wvu.edu/etd/1576

This Thesis is protected by copyright and/or related rights. It has been brought to you by the The Research Repository @ WVU with permission from the rights-holder(s). You are free to use this Thesis in any way that is permitted by the copyright and related rights legislation that applies to your use. For other uses you must obtain permission from the rights-holder(s) directly, unless additional rights are indicated by a Creative Commons license in the record and/ or on the work itself. This Thesis has been accepted for inclusion in WVU Graduate Theses, Dissertations, and Problem Reports collection by an authorized administrator of The Research Repository @ WVU. For more information, please contact researchrepository@mail.wvu.edu. 


\title{
Username and Password Verification through Keystroke Dynamics
}

\author{
Nick Bartlow \\ Thesis submitted to the \\ College of Engineering and Mineral Resources \\ at West Virginia University \\ in partial fulfillment of the requirements \\ for the degree of \\ Master of Science \\ in \\ Computer Science \\ Bojan Cukic, Ph.D., Chair \\ Arun Ross, Ph.D. \\ Lawrence Hornak, Ph.D. \\ Lane Department of Computer Science and Electrical Engineering \\ Morgantown, West Virginia \\ 2005
}

Keywords: Keystroke Dynamics, Behavioral Biometrics, Password Hardening, Replaceable Biometrics, Template Replaceability, Identity Verification.

Copyright (C)2005 Nick Bartlow 


\section{Abstract \\ Username and Password Verification Through Keystroke Dynamics}

Nick Bartlow

Most computer systems rely on usernames and passwords as a mechanism for access control and authentication of authorized users. These credential sets offer marginal protection to a broad scope of applications with differing levels of sensitivity. Traditional physiological biometric systems such as fingerprint, face, and iris recognition are not readily deployable in remote authentication schemes. Keystroke dynamics provide the ability to combine the ease of use of username / password schemes with the increased trustworthiness associated with biometrics. Our research extends previous work on keystroke dynamics by incorporating shift-key patterns. The system is capable of operating at various points on a traditional ROC curve depending on application specific security needs. A 1\% False Accept Rate is attainable at a 14\% False Reject Rate for high security systems. An Equal Error Rate of $5 \%$ can be obtained in lower security systems. As a username password authentication scheme, our approach decreases the penetration rate associated with compromised passwords by $95-99 \%$. 
To my family. 


\section{Acknowledgements}

- To Dr. Bojan Cukic- I would like to express my vast appreciation to my advisor and committee chair for all of the insight, guidance, patience, and encouragement he has bestowed upon me during my tenure as his graduate research assistant. In addition, I would like to particularly thank him for always having and sharing an abundance of new and interesting ideas every time I meander into his office. Unselfishly offering these jewels of information makes doing research for him a task in which boredom lies within the realm of sheer impossibility. Finally, I would like to thank him for maintaining his fruitful sense of humor and his casual attitude. The presence of these characteristics often help to turn work into "work." I'm not sure how well I'd be able to do work for someone lacking them. All this having been said, it is without exaggeration that I say I am honored and privileged to call myself his student.

- To Dr. Arun Ross $\&$ Dr.Larry Hornak- I would like to thank my remaining committee members for their willingness to always answer any occasional questions I may have had over the course of this work. Furthermore, I would like to show appreciation for their cooperation when continually pestered with never ending paperwork. Finally, I'd like to thank them for their undue patience in waiting for a labeled masochistic perfectionist to submit his work for review.

- To Dr. Tim Menzies- Thank you for ridiculous amount of information you passed on to us in your Data Mining class of Fall '03. At an absolute minimum, forcing us to learn shell scripting, and awk turned analyzing the data in this project from something that bordered on the level of intractability to a meer matter of pushing around some data with a few 20 line scripts. At the other end of the spectrum, the knowledge you shared undoubtedly had an effect on the success of this work. As you astutely pointed out, the "simplest technique imaginable" often delivers pretty good results.

- To Nate, Chris, Vivek, and Yan- Whether in the lab, at lunch, or back at the pad, thanks for always providing a backboard for me to bounce ideas off of. You are all selfless listeners who I always can count on to offer engaged and intelligent feedback to my incessant questioning. I am deeply indebted to you for this. 
- To Vivek and Andrés- Thanks for all the help with $\mathrm{IT}_{\mathrm{E} X}$. If it wasn't for you guys I'd never have been able to convert this thing in 3 days.

- Finally, to the Guinea Pigs- It goes without saying, none of this would ever have been possible without your patience and diligent participation. I thank you deeply for your time and help. Hopefully the result of your work will go beyond the scope of this individuals graduation. Finally, despite my best efforts to acknowledge you on an individual basis by name, I have been politely reminded that given the current state of the privacy regarding personally identifying data, I probably should not do so. I sincerely apologize that I am not able to include this individual recognition which is undoubtedly deserved. 



\section{Contents}

1 Introduction 1

1.1 Motivation . . . . . . . . . . . . . . . . . . . . . . . 1

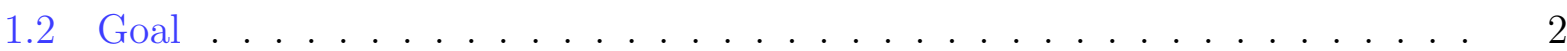

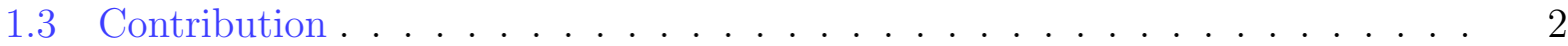

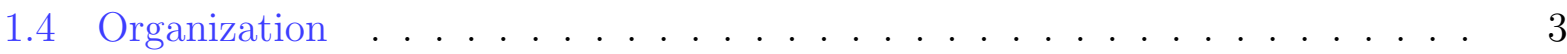

2 Literature Review $\mathbf{5}$

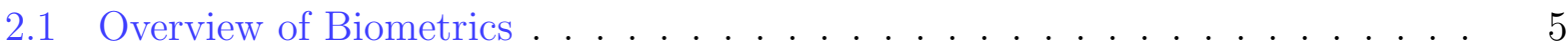

2.2 Performance Measures . . . . . . . . . . . . . . . . . . . . . . . . . 11

2.3 Machine Learning, Data Mining and Pattern Recognition . . . . . . . . . . . 14

3 Keystroke Dynamics as a Biometric $\quad 19$

3.1 Historical Perspective . . . . . . . . . . . . . . . . . . . . . . . . . . . . . . . 19

3.2 Keyboard Technology / Low-Level Interface . . . . . . . . . . . . . . . . 20

3.3 High Level Semantics . . . . . . . . . . . . . . . . . . . . . . . . . . . 21

3.4 Related Previous Work . . . . . . . . . . . . . . . . . . . . . 23

4 Experimental Design $\quad 27$

4.1 Overview \& Hypothesis . . . . . . . . . . . . . . . . . . . . . . . . . 27

4.2 Data Collection Results . . . . . . . . . . . . . . . . . . . . 34

5 Classification / Matching 35

5.1 Machine Learners . . . . . . . . . . . . . . . . . . . . . . 36

5.1 .1 OneR . . . . . . . . . . . . . . . . . . . . . . . . . 37

5.1 .2 NaiveBayes . . . . . . . . . . . . . . . . . . . . . . . . . . 38

5.1 .3 VotedPerceptron . . . . . . . . . . . . . . . . . . . . . . . . . 39

5.1 .4 LogitBoost . . . . . . . . . . . . . . . . . . . . . . . . . 40

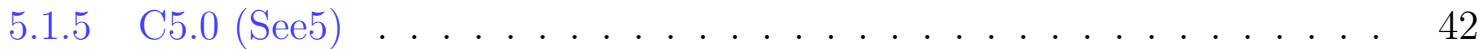


5.1 .6 Random Forests . . . . . . . . . . . . . . . . . . . . . 43

5.2 Inter-learner Performance Comparison . . . . . . . . . . . . . . 46

5.3 Short Password Performance vs. Long Password Performance . . . . . . . . 47

5.4 The Importance of Shift-Key Features . . . . . . . . . . . . . . . . . . . . . . . . . . . . . .

5.5 User Specific Voting Schemes . . . . . . . . . . . . . . . . 51

5.6 Per User System Performance . . . . . . . . . . . . . . . . . . . . 52

5.7 Overall System Performance . . . . . . . . . . . . . . . . 53

6 Discussion of Experimental Results $\quad 57$

7 Conclusion \& Future Work $\quad 61$

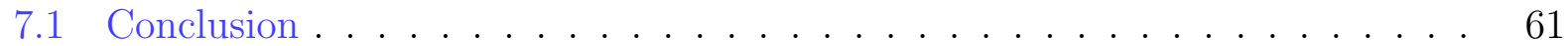

7.2 Future Work . . . . . . . . . . . . . . . . . 63

A Input Feature Descriptions $\quad 65$

$\begin{array}{ll}\text { B Weka Parameters } & 69\end{array}$

C Random Forest Complete Voting Scheme Results $\quad 71$

$\begin{array}{ll}\text { D User Graphs } & 77\end{array}$ 


\section{List of Figures}

2.1 Traditional Biometric System Layout . . . . . . . . . . . . . . . . . . 10

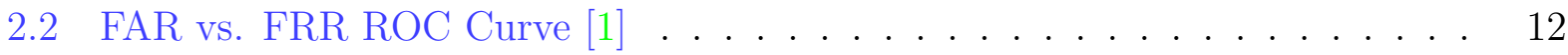

2.3 Relationship between Machine Learning, Data Mining, and Pattern Recognition 16

3.1 Switch types found in modern keyboards [2] . . . . . . . . . . . . . . . 20

4.1 System Registration Section . . . . . . . . . . . . . . . . . . 31

4.2 Genuine Input Section . . . . . . . . . . . . . . . . . . . . . . . . . . . . . . . . . . . . . . .

4.3 Genuine Input Section . . . . . . . . . . . . . . . . . . . . . 32

5.1 Differing RF Voting Schemes for Optimization of EER Across Users . . . . . 52

5.2 Overall System Performance ROC Curve . . . . . . . . . . . . . . . 54

5.3 Classification Accuracy vs. Random Forests Voting Scheme . . . . . . . . . . 56 



\section{List of Tables}

3.1 Previous Work . . . . . . . . . . . . . . . . . . . . . . 24

4.1 Feature Vector Collected for Each Input Sequence . . . . . . . . . . . . . 33

5.1 OneR's Overall Performance . . . . . . . . . . . . . . . . . . . . . 38

5.2 NaiveBayes's Overall Performance . . . . . . . . . . . . . . . . . . . . 39

5.3 VotedPerceptron's Overall Performance . . . . . . . . . . . . . . . . . . 40

5.4 LogitBoost's Overall Performance . . . . . . . . . . . . . . . . . . . . . . 41

5.5 C5.0's Overall Performance. . . . . . . . . . . . . . . . . . . . . 42

5.6 Random Forest Overall Performance with 0.55-0.45 Genuine Imposter Voting Scheme... . . . . . . . . . . . . . . . . . . 44

5.7 Random Forest Overall Performance with 0.25-0.75 Genuine Imposter Voting Scheme. . . . . . . . . . . . . . . . . . . . 45

5.8 Random Forest Overall Performance with 0.75-0.25 Genuine Imposter Voting Scheme. . . . . . . . . . . . . . . . . . . . 45

5.9 Learner Performance Comparison Through 95\% Confidence Intervals . . . . 46

5.10 Statistical Significance of Difference in Performance Between Short and Long Passwords Across Learners . . . . . . . . . . . . . . . . . . . . 48

5.11 Statistical Significance of Difference in Performance Between Short and Long Passwords Across Random Forests . . . . . . . . . . . . . . . . . . . 48

5.12 Feature Importance in Input Sequences . . . . . . . . . . . . . . . 50

5.13 System Performance Per User . . . . . . . . . . . . . . . . . . . . . 53

5.14 System Password Hardening Effect . . . . . . . . . . . . . . . 55

C.1 Random Forest Overall Performance with 0.05-0.95 Genuine Imposter Voting Scheme. . . . . . . . . . . . . . . . . . . . 71

C.2 Random Forest Overall Performance with 0.10-0.90 Genuine Imposter Voting Scheme . . . . . . . . . . . . . . . . . . . . . 
C.3 Random Forest Overall Performance with 0.15-0.85 Genuine Imposter Voting Scheme . . . . . . . . . . . . . . . . . . . .

C.4 Random Forest Overall Performance with 0.20-0.80 Genuine Imposter Voting Scheme . . . . . . . . . . . . . . . . . . . . .

C.5 Random Forest Overall Performance with 0.25-0.75 Genuine Imposter Voting Scheme . . . . . . . . . . . . . . . . . . . .

C.6 Random Forest Overall Performance with 0.30-0.70 Genuine Imposter Voting Scheme . . . . . . . . . . . . . . . . . . . .

C.7 Random Forest Overall Performance with 0.35-0.65 Genuine Imposter Voting Scheme . . . . . . . . . . . . . . . . . . . .

C.8 Random Forest Overall Performance with 0.40-0.60 Genuine Imposter Voting Scheme . . . . . . . . . . . . . . . . . . . . .

C.9 Random Forest Overall Performance with 0.45-0.55 Genuine Imposter Voting Scheme . . . . . . . . . . . . . . . . . . . .

C.10 Random Forest Overall Performance with 0.50-0.50 Genuine Imposter Voting Scheme . . . . . . . . . . . . . . . . . .

C.11 Random Forest Overall Performance with 0.55-0.45 Genuine Imposter Voting Scheme . . . . . . . . . . . . . . . . . . . .

C.12 Random Forest Overall Performance with 0.60-0.40 Genuine Imposter Voting Scheme . . . . . . . . . . . . . . . . . . .

C.13 Random Forest Overall Performance with 0.65-0.35 Genuine Imposter Voting

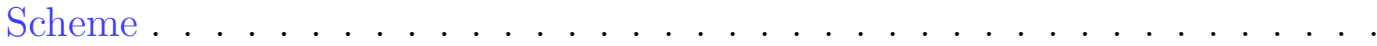

C.14 Random Forest Overall Performance with 0.70-0.30 Genuine Imposter Voting Scheme . . . . . . . . . . . . . . . . . . .

C.15 Random Forest Overall Performance with 0.75-0.25 Genuine Imposter Voting Scheme . . . . . . . . . . . . . . . . . . .

C.16 Random Forest Overall Performance with 0.80-0.20 Genuine Imposter Voting Scheme . . . . . . . . . . . . . . . . . . . . .

C.17 Random Forest Overall Performance with 0.85-0.15 Genuine Imposter Voting Scheme . . . . . . . . . . . . . . . . . . . .

C.18 Random Forest Overall Performance with 0.90-0.10 Genuine Imposter Voting Scheme . . . . . . . . . . . . . . . . . . .

C.19 Random Forest Overall Performance with 0.95-0.05 Genuine Imposter Voting Scheme . . . . . . . . . . . . . . . . . 76

72

72

72

72

73

73

73

74

74

74

75

75

75

75

76

76 


\section{Chapter 1}

\section{Introduction}

\subsection{Motivation}

With the onset of the Internet, the importance of identification technologies has increased rapidly over the last decade. The amount of information that is accessed and transmitted on a daily basis is phenomenal. Furthermore, much of this information is sensitive in nature and should only be accessible by authorized users only. Systems with this burden often rely on usernames and passwords to administer such controlled access. Relying solely on passwords for authentication raises many security issues. The most important issue is that when passwords are compromised (stolen), the intruder necessarily has full access to the rights and information of the authorized user. The field of biometrics has offered an alternative to password based authentication and makes forging entry into systems much more difficult when compared to passwords. Unfortunately, biometric authentication is often not readily

deployable in remote environments such as web access as the enrollment and verification 
processes often require strict supervision and various hardware devices that a typical user does not own. Keystroke dynamics may be a way to combine the usability of username and password schemes with the benefits of biometric systems at minimal cost to system administrators and users.

\subsection{Goal}

The goal of this study is to establish the viability of keystroke dynamics with username and password input as a possible method of hardening authentication credentials. It should also result in a method which allows for a biometric system to be readily deployable both in an unsupervised and remote fashion. Finally, the study will attempt to establish the difference in performance of the biometric system associated with two significantly different types of passwords.

\subsection{Contribution}

This study results in numerous contributions to fields of keystroke dynamics, biometrics, and information security. In terms of keystroke dynamics, it is the first study in that includes the significance of shift-key behavior on matching performance. It also represents by far the most statistically significant study in terms of data collected. Finally, it presents a novel approach to matching keystroke dynamics input in terms of the algorithm applied. In terms of biometrics, the study represents another look into the viability of keystroke dynamics as a potential biometric. Finally, this study will offers useful knowledge to information security 
experts as it provides an easily implemented approach to strengthening password-based systems through the use of an unobtrusive and replaceable biometric.

\subsection{Organization}

The remaining sections of this thesis are broken up as follows. Chapter 2 represents a literature review providing introductions into biometrics, performance measures within biometrics, machine learning, data mining, and pattern recognition. Chapter 3 covers the topic of keystroke dynamics including previous work, technological aspects, and semantics used in our study. Chapter 4 outlines the experimental design with detailed description of how it was implemented. Chapter 5 focuses on the performance of the system using various algorithms for matching / classification while also providing insight into the difference between the two different types of passwords. Chapter 6 offers considerations and limitations of the system regarding its benefits and its ability to be deployed as a real-world application. Finally

Chapter 7 provides the conclusions that are made as a result of the study and how they apply to the related fields. 



\section{Chapter 2}

\section{Literature Review}

The scope of this literature review is to familiarize the reader with three concepts directly related to this project; an overview of biometrics, performance measures within biometrics, and machine learning.

\subsection{Overview of Biometrics}

The problems of identification and verification of individual identity have existed throughout the course of human history. Naturally various means have been used to solve these problems. These means have traditionally been broken down into four main categories [3][4]:

1. What you have

2. What you know

3. What you are 
4. Where you are

The third category encompasses the field of biometrics as we know it today. What you are can be thought of as an embodiment of various body characteristics and actions [4]. Probably the first formal account of using such characteristics for an identity scheme occurred in 14th century China when fingerprints were used as a form of signature [5]. Informally, it is easy to imagine visual identification schemes existed even before the scope of written history. Current schemes include an extremely vast range of characteristics spanning anywhere from the aforementioned palm prints to DNA related technologies. It should be noted that historically biometrics have been used in identity schemes typically in a hands-on, manual fashion. On the other hand, the current state of the field would define biometrics as automated methods of establishing identity based on one or more physiological / behavioral traits [6]. This definition implies a difference between a biometric system and a biometric characteristic or trait.

With a virtually infinite amount of characteristics making up a human being, a framework has been devised to determine which characteristics can be considered valid as legitimate biometrics [4]. A given physical or behavioral characteristic must satisfy to some degree all of the following seven qualities in order to be considered a potential biometric characteristic / trait.

1. Universality- The characteristic should exist for each person in a given population. For instance, typically all individuals have fingerprints. That having been said, some individuals have lost digits in accidents or have substantially injured them to the point that prints cannot be extracted. 
2. Distinctiveness- The characteristic should be sufficiently different between any two individuals within the population. For instance, height is a characteristic that can serve as an identifying factor over very small populations but clearly is not sufficient for typical biometric systems [4].

3. Permanence- The characteristic should not vary significantly over a given period of time. For example, a face of a human at age 30 may vary significantly compared to the face of the same human at age 60 [4].

4. Collectability- There must be a ways to both measure the characteristic quantitatively and acquire the characteristic automatically. It is not enough to say, "This iris looks like that iris." Formal measurements must be extractable. Furthermore, the manner in which these quantitative measures are acquired must be automatic; manual extraction cannot occur in modern biometric systems.[4].

5. Performance- The degree in which the system based on the characteristic(s) can perform relative to desired levels of identification / recognition accuracy and speed [4].

6. Acceptability- The level in which the population accepts the use of the characteristic. DNA is extremely unique but other factors such as sample collection and privacy concerns pose problems in terms of user acceptance [4].

7. Circumvention- The degree in which a system based on the potential characteristic(s) can be easily fooled through fraudulent methods [4].

Clearly no potential characteristic can satisfy all qualities to the greatest degree. The degree in which a candidate characteristic satisfies these qualities often dictates which type of 
biometric system / application it can be used for, i.e. personal use, low security commercial access, high security access, etc

As mentioned earlier, there is an inherent difference between physiological biometrics and behavioral biometrics. Physiological biometrics are characteristics defined typically by anatomical means in that they are related to the structure of the organism in question. Examples of such physiological biometrics include but are not limited to:

- DNA

- Ear

- Face

- Fingerprint

- Hand Geometry

- Palmprint

- Hand Vein Print

- Iris

- Retina

- Footprint

- Odor 
Traditionally, it is said that physical biometrics are irreplaceable, in that anatomical structures such as those listed are not easily removed or fabricated. Behavioral biometrics unlike physiological are based on human action or the way one behaves. Examples of such biometrics include but are not limited to:

- Gait (the way one walks, runs, etc)

- Signature

- Face

- Speech / Voice

- Keystrokes

Typically these behaviors are the result of many years of acclimation and or training. For example, the way you walk or the way you sign your name; it can be said that the current state of either behavior is the culmination of many years of practice / experience. On the other hand, it has been said that behavioral biometrics offer a degree of replaceability that physiological biometrics do not. For instance, in terms of a person to person comparison, the way an individual says one word may be fundamentally different from the way he says a different word. Furthermore, although a time intensive process, it is said that individuals may be able to change such behavioral characteristics given the appropriate desire / commitment. For example, one could reinvent the way she signs her signature, it would take a lot of practice, but it is clearly a possible. The final aspect of this overview to consider is the layout of a generic biometric system. The layout of a traditional biometric system as outlined by Ratha [7] is pictured in Figure 2.1. Within this layout, there are three main 


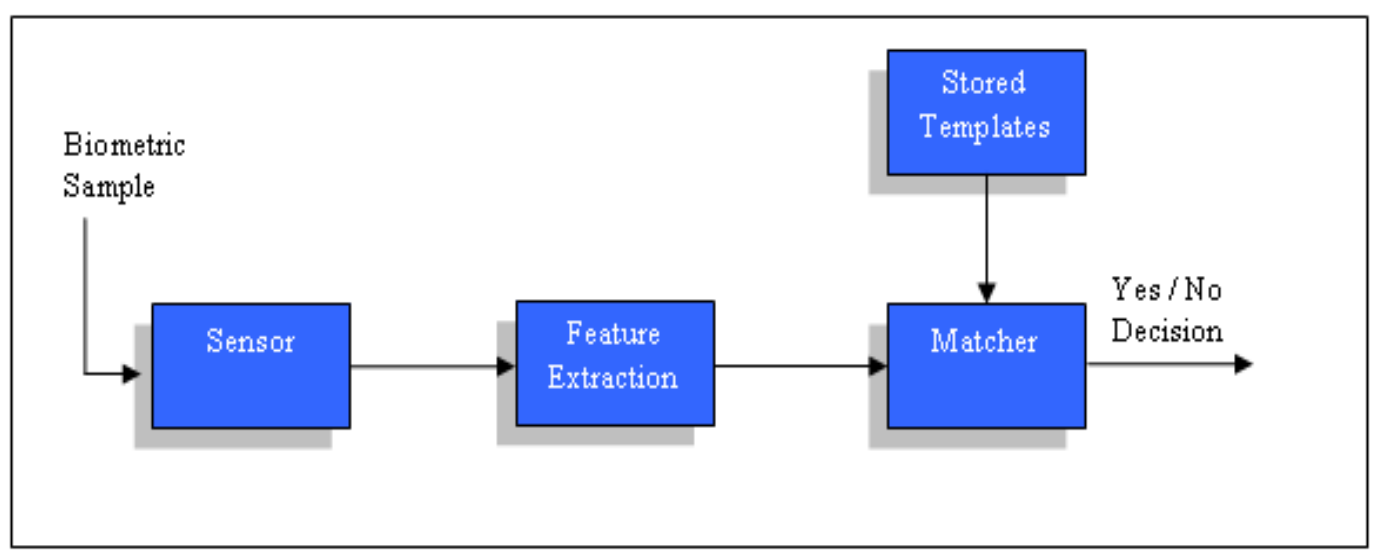

Figure 2.1: Traditional Biometric System Layout

blocks; sensor, feature extraction, feature and matching. The sensor block obtains raw data representing the biometric sample. After receiving the raw data typically in the form of an image, the feature extraction block removes selected information that represents the uniquely identifying features of the biometric sample. During enrollment, these uniquely identifying features are typically stored in the system database as templates. At this point, the extracted features are passed into the matching block. Within this block matching feature matching is performed either against one or more templates of one user (verification) or against one or more templates of all users (identification). It should be noted that biometric systems in verification mode verify that a person is the identity they claim to be (one to one). In identification mode, a user provides no claim and the biometric system attempts to match him / her to someone already enrolled in the database (one to many). Another mode not formally addressed in basic diagrams is negative recognition in which the biometric system assures the individual who provided the sample is not a member of a given database. In the last block, a matching score is produced and the decision of "yes" or "no" is determined 
based on the relationship between the match score and the threshold (minimum / maximum score acceptable for a match).

\section{$2.2 \quad$ Performance Measures}

Although talk of performance measures in biometric systems can easily be included in an overview it was believed that the topic is significant enough to merit a complete section on its own. Performance of biometric systems is typically measured in terms of various error rates, namely False Accept Rate (FAR) and False Reject Rate (FRR). The FAR refers to the errors the biometric system makes in which it incorrectly matches the features of the biometric sample presented with those in the system database, smartcard, etc. In statistics, this is referred to as a Type II error. The FRR refers to the errors the biometric system makes in which it incorrectly does not match the biometric sample presented with those in the system database, smartcard, etc In statistics, this is referred to as a Type I error. It is common practice to plot the FARv s. FRR in a graph referred to as a Receiver Operating Characteristic (ROC) curve which allows for analysis of where a biometric stands based on the applications requirements. Figure 2.2 shows such a ROC curve with different application scenarios noted. Occasionally some groups refer to FAR and FRR as False Non-Match Rate (FNMR) and False Match Rate (FMR) respectively.

Besides the traditional FAR vs. FRR measures, there are also a series of singleton measures that can be used to assess the performance of biometric systems. These measures include the Equal Error Rate (EER), d-prime, and many other off-shoots of the d-prime 


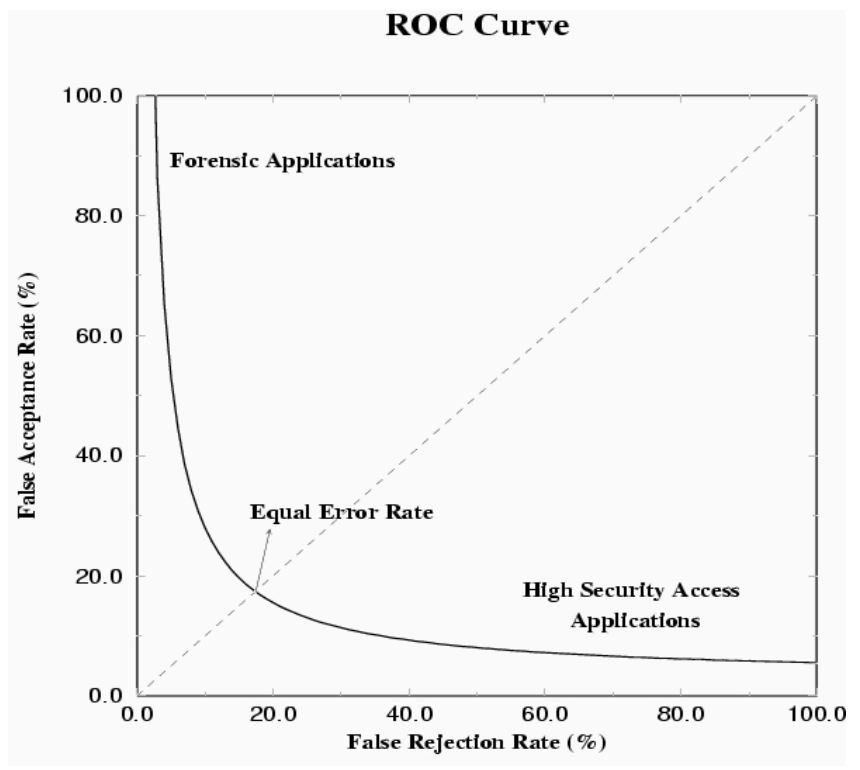

Figure 2.2: FAR vs. FRR ROC Curve [1]

measure. The EER is relatively simple to understand in that is it the point in the ROC curve in which the FAR equals the FRR. Although there may not be a specific point in which this precisely occurs in all data sets for a biometric system, it is typically estimated by plotting the line $\mathrm{Y}=\mathrm{X}$ in a conventional ROC curve. In Figure 2.2, the system EER falls just below 20\%. If a precise measurement of EER is required, Equation 2.2 can be applied.

$$
\begin{gathered}
E E R=\frac{F A R_{t *}+F R R_{t *}}{2} \\
t *=M I N_{t}\left|F A R_{t}-F R R_{t}\right| \\
\mathrm{t}=\text { threshold }
\end{gathered}
$$


D-prime offers a parametric distribution based singleton performance metric. Taking in the mean and standard deviations of both the imposter and genuine distributions one can determine the degree of overlap of the two classes. Equation 2.2 displays the equation to arrive at the d-prime measure for a given system.

$$
\begin{gathered}
d^{\prime}=\frac{\left|\mu_{G}-\mu_{I}\right|}{\sqrt{\sigma_{G}{ }^{2}+\sigma_{I}^{2}}} \\
G=\text { Genuine, } I=\text { Imposter }
\end{gathered}
$$

It should be noted that this measure is most applicable if both distributions are Gaussian / normally distributed. With this measure, the higher values indicate a greater degree of separation between genuine and imposter distributions.

All the previously described metrics have deal with the "positive view" aspects of performance measures within biometric systems. "Negative view" performance measures often overlooked by casual observers of biometric systems and often notoriously excluded from commercial product spec sheets and white papers. Specifically, Failure to Enroll (FTE) and Failure to Acquire (FTA) rates are often used to bloat the more common observed FAR and FRR measures previously mentioned. The FTE rate measures the percentage of individuals who are not able to be enrolled in the system for any number of reasons. Typically, this occurs if an individual does not have the biometric (loss of finger / eye) or has a severely damaged biometric that the system cannot process during enrollment. The FTA rate mea- 
sures the percentage of time in which the biometric system cannot obtain the raw biometric data of an enrolled user during verification / identification modes. This may occur due to undue noise in the system such as loud background sound in a speaker recognition system or an extremely dry winter day in a capacitive fingerprint system. As mentioned before, commercial entities often like to conveniently not include these measures in product spec sheets while internally relying on them to bolster FAR / FRR measures. It is not hard to imagine how one could simply exclude individuals with sub-par biometrics samples either at the enrollment stage or at verification / identification stages by simply not allowing enrollment or continuation into matching blocks of the biometric system. Clearly this practice has positive effects on FAR / FRR rates, which often taken singularly, are not indicative of the overall performance of the biometric system. Therefore, the overall matching performance of a biometric system should minimally be measured by four metrics; FAR, FRR, FTE, and FTA.

\subsection{Machine Learning, Data Mining and Pattern Recog- nition}

Although subtly different, generally speaking, the fields of Machine Learning, Data Mining and Pattern Recognition all deal with fully or semi-automated methods of describing structural patterns within data. The idea of describing structural patterns of data can be thought of as obtaining information about data in one of many ways; explanation, interpretation, validation, and prediction. Formal definitions shed more light on the three fields [8]. 
- Machine Learning- An area of artificial intelligence concerned with the development of techniques which allow computers to change behavior in a way to improve future performance $[8][9]$.

In "Data Mining," Witten and Frank subsequently note the difference between learning and training lies in the intent of the learning entity and the ownership of learning purpose (teacher in training, learner in learning) [8].

- Data Mining- Data processing using sophisticated data search capabilities and statistical algorithms to discover patterns and correlations in large preexisting databases; a way to discover new meaning in data [10].

The Wikipedia definition suggests a synonymous relationship between data mining and Knowledge Discovery in Databases (KDD) [11]. This thought is supported by Witten and Frank with particular stress on importance of knowledge discovery in the form of comprehensible models [8].

- Pattern Recognition- A branch of artificial intelligence concerned with the classification or description of observations. Pattern recognition aims to classify data (patterns) based on either a priori knowledge or on statistical information extracted from the patterns. The patterns to be classified are usually groups of measurements or observations, defining points in an appropriate multidimensional space [12].

This definition suggests a noted difference between data-mining in that although a description of observations is attained, the resulting descriptive and or predictive model need not 


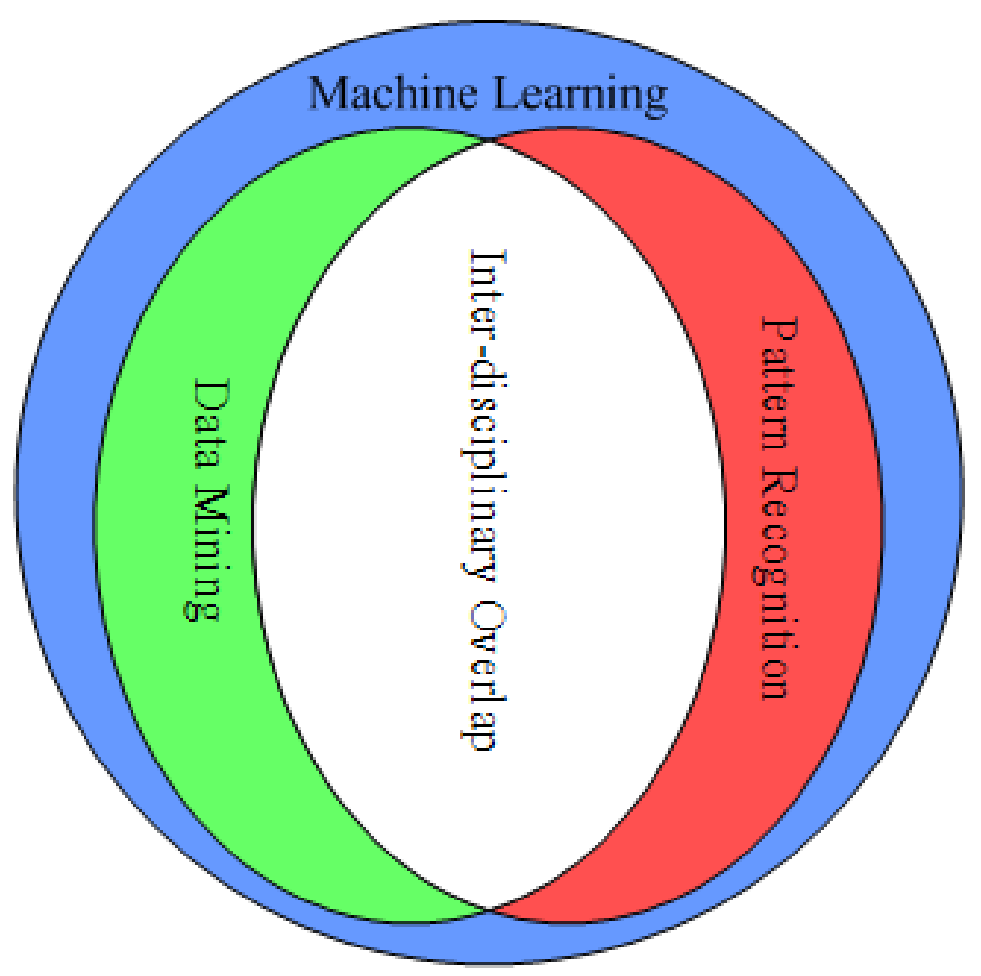

Figure 2.3: Relationship between Machine Learning, Data Mining, and Pattern Recognition

be readily comprehensible to humans. This may be implied by the phrase "appropriate multidimensional space" as most humans have difficulties interpreting dimensionality beyond three levels such as 4-D or 5-D space. Witten and Frank offer the same sentiment when referring to neural networks [8]. Figure 2.3 offers one final attempt to allow an individual to examine how the three fields relate to each other. It indicates that data-mining and pattern recognition are subsets of the overall field of machine learning. Furthermore, the figure shows how data mining and pattern recognition are not completely independent of each other.

These concepts were used within our keystroke analysis study with the hopes of attaining 
an acceptable predictive solution to the two-class classification problem (genuine, imposter) presented by the verification mode of the potential biometric system. In this regard, the pattern recognition subset of machine learning was called upon. Besides the desired predictive mechanism measured by performance, it was also believed that some of the machine learning algorithms employed could offer comprehensible models explaining the structure of the two-class problem. In that regard, the data mining subset of machine learning was called upon. Therefore this study seems to be holistic relative to the three fields and would necessarily fall within the white, central region of 2.3. 



\section{Chapter 3}

\section{Keystroke Dynamics as a Biometric}

\subsection{Historical Perspective}

Keystroke Patterns have been used as a biometric since the early 1900's in the days of WWI. During the war, the French used listening posts in which operators were able to recognize the "fist" of enemy radio operators communicating in Morse code. These trained individuals would learn to recognize operators by differing lengths of pauses, dots and slashes, and varying transmission speeds. This intelligence subsequently allowed the French to establish the identity of entities such as enemy battalions [13]. Although this communication did not include the use of keyboards as we know them today, the increased complexity of today's keyboards undeniably allows for even more unique pattern development. 


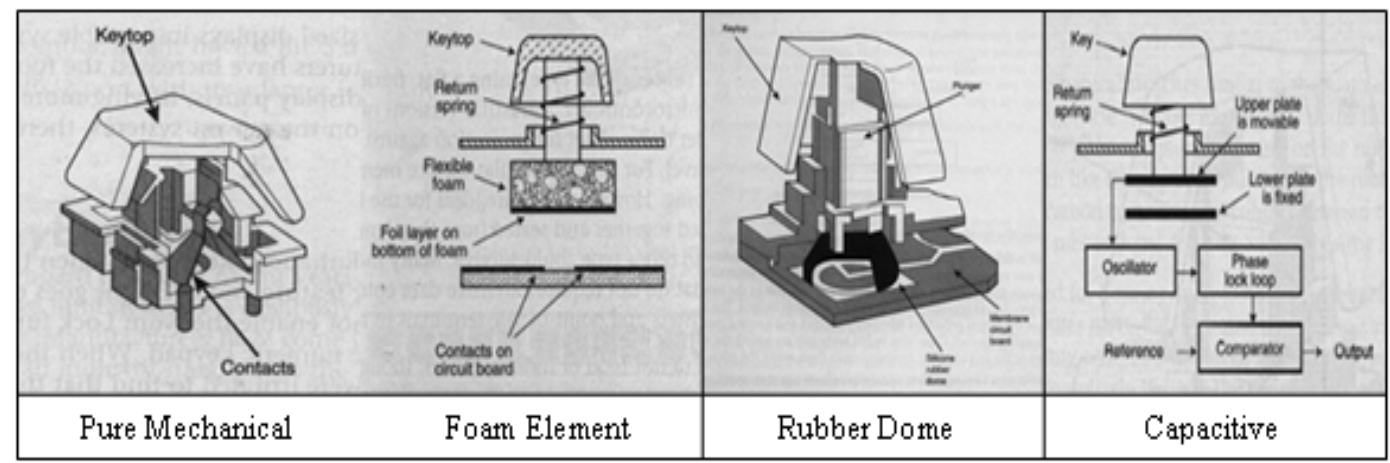

Figure 3.1: Switch types found in modern keyboards [2]

\subsection{Keyboard Technology / Low-Level Interface}

In order to fully understand the terminology to be used in this paper, a basic understanding of keyboard technology at its lowest level is necessary. This section attempts to outline said technology. The first point of interest in low level keyboard technology is the different types of keyboard layouts found in computers today. There are three main types; 101/2 key layouts, 104 key layouts, and laptop layouts [2]. Laptop layouts are not standardized and have a wide range of number of keys in their layouts so no value is specified. The differences in these layouts are not of particular significance as the ability to input all printable and non-printable control characters used in this study are possible regardless of the particular keyboard layout of a given user.

Delving beyond layout, there are four different kinds of switch technology used in keyboards today; pure mechanical, foam element, rubber dome, and membrane [2]. Figure 3.2 provides a visual representation of these four switch types. Each switch type has various character- 
istics such feel, durability, price, etc That having been said, these characteristics are not of importance relative to this project. No matter the key switch technology chosen, when a key is depressed a degree of "bounce" is present. Bounce is the effect when the contact device rapidly engages and disengages over an extremely short period of time [2]. Keyboards, either external to desktop PC's or internal to laptops are computers in their own right as they contain a microprocessor, RAM, and sometimes ROM. Using their processors and controllers, they filter out the difference between bounce and two successive keystrokes. Each stroke therefore consists of two events, when the plates are engaged and when the engagement is released or disengaged. Scan codes resulting from these events are sent from the controller in the keyboard to the event handler in the BIOS of the PC itself [2]. Scan codes are recorded by the processor based on a matrix composed of all the keys on the keyboards. The keyboard matrix operates on a buffer that allows for the processing of simultaneous keystroke events. As mentioned before, when a key is pressed down the plates become engaged, it is at this point that the keyboard processor sends a "make code" encoded as a hex value to the PC. The make code can be thought of as including both the key engaged and various other state flags indicating if / how the key was modified by any of the various control keys such as shift, alt, etc Once the key disengages, a corresponding "break code" is sent to the PC [2]. These ideas form the basis of keyboard technology at its lowest-level.

\subsection{High Level Semantics}

The data that will be collected for this project is directly related to the various terminologies found in the previous section. However, for reasons of simplicity, we map the somewhat 
terse low level terminology into more intuitive higher level events. This section focuses on establishing an understanding of the semantics of these high level events. The basis of all features included in keystroke dynamics is founded on the keystroke event and the associated "make code" / "break code" correlation described in the previous section. Instead of dealing with terms like "make code," "disengagement", etc, we define and use the following terms of interest which are much more intuitive.

1. KeyDown- The event that fires when a key is pressed down. This corresponds to the event of the keyboard processor sending the PC a "make code." It should be noted that this event will continually fire until the key being depressed is released. The speed at which the KeyDown event fires while a key is depressed is referred as the "repeat rate." This is a user customizable property in virtually all operating systems.

2. KeyUp- The event that fires when a currently depressed key is subsequently released.

3. Keystroke- The combination of an initial KeyDown event and the corresponding KeyUp event.

4. Hold Time- the length of time between an initial KeyDown event and the corresponding KeyUp event.

5. Delay (latency)- The length of time between two successive keystrokes. It should noted that this time can be positive or negative (overlapping strokes). 


\subsection{Related Previous Work}

As mentioned in the historical perspective, keystroke dynamics have been around, in some form, since the early 1900's. Keystroke dynamics as known today are, on the other hand, a significantly more youthful technology having first come about in a work by Gaines and Lisowski in the early 1980's [13]. Although not claiming to be exhaustive, this section attempts to provide a thorough overview of the related previous work that has been conducted since 1980 including academic research, government patents, and private sector commercial technology. The chronologically ordered overview focuses on four major sections of each study; the primary keystroke feature(s) and algorithm employed, input requirements for a given sequence, scope of the study in terms of users and sequences gathered, and the performance relative to the scope of the study. For the purposes of easy comparison, the overview is provided as Table 3.1. The table provides a look into the field both during its infancy and at the current state of the art. Instead of laboring over the particular intricacies of each individual work, it seems more beneficial to point out both milestones and overall trends that developed as the field matured. As noted before, the initial work was performed by Gaines and Lisowski in 1980 [14]. It was at this time that the advent of the use of interkey latency / delay was employed. Regarding features collected, we see that no significant additions occur until Obaidat and Sadoun in 1997 when they introduced key hold times as another feature of interest [20]. Over the last two decades we see a plethora of matching algorithms offered ranging anywhere from statistical based techniques including t-tests, means, and standard deviations, to conventional distance metrics such as Euclidean and Mahalanobis, to a Machine Learning algorithms such as Neural Networks and Perceptron [6]. It should also be 


\begin{tabular}{|c|c|c|c|c|}
\hline Work & Feature(s) / Algorithm & Input & Scope & Performance \\
\hline $\begin{array}{l}\text { Gaines \& Lisowski } \\
(1980)[14]\end{array}$ & $\begin{array}{l}\text { Latency between } 87 \text { lowercase digraphs } \\
\text { using sample t-tests }\end{array}$ & $\begin{array}{l}300-400 \text { word pas- } \\
\text { sage } 2 \text { times }\end{array}$ & $\begin{array}{l}7 \text { secre- } \\
\text { taries }\end{array}$ & $\begin{array}{l}\text { FAR } 0 \%(0 / 55) \text { FRR } \\
4 \%(2 / 55)\end{array}$ \\
\hline Garcia (1986) [15] & $\begin{array}{l}\text { Latency between } 87 \text { lowercase digraphs } \\
\text { and space key \& Complex Discrimina- } \\
\text { tion using Mahalanobis distance func- } \\
\text { tion }\end{array}$ & $\begin{array}{l}\text { Individual's name } \\
\& \quad 1000 \text { common } \\
\text { words } 10 \text { times each }\end{array}$ & $\mathrm{N} / \mathrm{A}$ & $\begin{array}{l}\text { FAR } 0.01 \% \quad(\mathrm{~N} / \mathrm{A}) \\
\text { FRR } 50 \%(\mathrm{~N} / \mathrm{A})\end{array}$ \\
\hline $\begin{array}{l}\text { Young \& Hammon } \\
\text { (1989) [16] }\end{array}$ & $\begin{array}{l}\text { Plurality of features including: digraph } \\
\text { latencies, time to enter selected number } \\
\text { of keystrokes and common words using } \\
\text { Euclidean distance }\end{array}$ & $\mathrm{N} / \mathrm{A}$ & $\mathrm{N} / \mathrm{A}$ & $\mathrm{N} / \mathrm{A}$ \\
\hline $\begin{array}{lcl}\text { Joyce } & \& & \text { Gupta } \\
(1990) & {[17]}\end{array}$ & $\begin{array}{l}\text { Digraph latencies between reference } \\
\text { strings using mean and standard devi- } \\
\text { ation of latency distance vectors }\end{array}$ & $\begin{array}{l}\text { Username, pass- } \\
\text { word, first name, } \\
\text { last name } 8 \text { times } \\
\text { each }\end{array}$ & $\begin{array}{l}33 \text { users } \\
\text { of varying } \\
\text { ability }\end{array}$ & $\begin{array}{lrr}\text { FAR } & 0.25 \% & (2 / 810) \\
\text { FRR } & 16.36 \% & (27 / 165)\end{array}$ \\
\hline $\begin{array}{l}\text { Obaidat \& Mac- } \\
\text { chiarolo (1993) [18] }\end{array}$ & $\begin{array}{l}\text { Digraph latencies between reference } \\
\text { strings using Neural Networks }\end{array}$ & $\begin{array}{l}15 \text { character phrase } \\
20 \text { times each }\end{array}$ & 6 users & $97 \%$ overall accuracy \\
\hline $\begin{array}{l}\text { Bleha \& Obaidat } \\
(1993)[19]\end{array}$ & $\begin{array}{l}\text { Digraph latencies between of strings us- } \\
\text { ing Perceptron algorithm (50\% test / } \\
50 \% \text { train) }\end{array}$ & Username ? Times & 24 users & FAR $8 \%$ FRR $9 \%$ \\
\hline $\begin{array}{l}\text { Obaidat \& Sadoun } \\
(1997)[20]\end{array}$ & $\begin{array}{l}\text { Digraph latencies and key hold times } \\
\text { using multiple machine learning algo- } \\
\text { rithms }\end{array}$ & $\begin{array}{l}\text { Username } 225 \\
\text { times / day for } 8 \\
\text { weeks }\end{array}$ & 15 users & $\begin{array}{l}\text { FAR } 0 \%(\mathrm{~N} / \mathrm{A}) \text { FRR } \\
0 \%(\mathrm{~N} / \mathrm{A})\end{array}$ \\
\hline $\begin{array}{l}\text { Monrose, Weiter, \& } \\
\text { Wetzel (2001) [21] }\end{array}$ & $\begin{array}{l}\text { Digraph latencies and key hold times, } \\
\text { algorithm employed is unclear }\end{array}$ & $\begin{array}{l}8 \text { character pass- } \\
\text { word }\end{array}$ & 20 users & $\begin{array}{l}\text { FAR \%(N/A) FRR } \\
45 \%(\mathrm{~N} / \mathrm{A})\end{array}$ \\
\hline $\begin{array}{l}\text { Bergadano, } \\
\text { Gunetti, \& Pi- } \\
\text { cardi }(2002)[22]\end{array}$ & $\begin{array}{l}\text { Trigraph duration using degree of dis- } \\
\text { order }\end{array}$ & $\begin{array}{l}683 \text { character text } 5 \\
\text { times }\end{array}$ & 44 users & $\begin{array}{lr}\text { FAR } & 0.04 \% \\
(1 / 10,000) & \text { FRR } \\
4 \%(\mathrm{~N} / \mathrm{A}) & \\
\end{array}$ \\
\hline BioPassword[23] & Patented by Young (1989)[16] & $\begin{array}{l}\text { Username } \\
\text { Password }\end{array}$ & $\mathrm{N} / \mathrm{A}$ & $\mathrm{N} / \mathrm{A}$ \\
\hline
\end{tabular}

Table 3.1: Previous Work

noted that there is at least one noted trend that occurs over that time period and continues through present day. Initial works required extremely demanding input requirements in terms of length of characters ranging from large passages and word lists (on the order of 100's and sometimes 1000's of characters) whereas more recent work has attempted to minimize such input requirements relying only on username and or passwords (on the order of 10's of characters).

There is also a relatively wide range in performance over the two decades with published 
FAR ranging from 0-8\% and FRR ranging from 0-45\%. Although these are ranges are indeed wide, some studies seem to offer perfect, if not near perfect performance in both measures. When considering these studies, it is highly important to understand the relationship between FAR / FRR performance and the scope of the study. There are notable deficiencies across the board in that virtually all studies seem to have been conducted over extremely small user populations or have only collected a limited amount of inputs per user. Most of the papers admit that these deficiencies have a high impact on conclusions that can be drawn from the performance results due to the limited amount of data collected. 



\section{Chapter 4}

\section{Experimental Design}

\subsection{Overview \& Hypothesis}

As mentioned in the previous section, several keystroke dynamics experiments have taken place since the early 1980's. The results of many experiments seem to support conclusions in defense of using keystroke dynamics as a biometric. We have decided to use the following characteristics to judge the results of such an experiment:

1. Performance- Traditionally, performance is measured in biometric systems in terms of error rates including FAR, FRR, and EER. Other measures such as d-prime and d-prime variants can also be considered.

2. Number of Users- One consideration of paramount importance in biometrics is uniqueness. Uniqueness can only be established over large, statistically significant databases including many users. 
3. Size of Data Set- Both the number of users and the number of trials per user in terms of input sequences must be statistically significant.

4. Cost of Enrollment- Usability is also of paramount importance for biometric systems. Besides traditional methods of minimizing FRR's, it is also important to minimize cost of enrollment both in terms of time and supervision.

5. Algorithm Employed- Another obvious consideration that is not completely orthogonal to the previous characteristics is the algorithm deployed. The validity and strength of the algorithm in terms of time complexity and resource efficiency is very important in biometric systems.

The design of our case study was based on a goal to meet all of these considerations to the highest degree possible. Therefore, we decided that in order to facilitate a large user database the collection of keystroke features should be web-based. Based on this decision, front-end client-side Java applets were developed in the NetBeans Integrated Development Environment (IDE) and designed to run within web browsers (Mozilla Firefox, Internet Explorer, Netscape) using the Sun Java Console. This offered both a tested open source platform as well as the bulk of the computation to occur in the client computer. On the server side, a MySQL database (also an open source product) was used to house the data. Therefore, once client-side computation was finished, the input was entered into the database via the already established client-server connection.

The system required the user to input only username and password fields which ensured minimal input in both the registration / participation sections. It should be noted that 
this design allowed for user participation to be "completely unsupervised" in that the only guidance / direction users received was in the form of written instructions and short video clips.

Related work seemed to indicate that algorithms employing inter-key latencies or delays combined with hold times appear to be to be most successful when given minimal data per sequence. The most notable difference in the algorithm we developed in this project is that shift key behavior was not only considered but highly emphasized. We hypothesized that shift key behavior varies greatly across users. For instance, a trained professional will use the left shift key to modify characters typed with the right hand and the right shift key to modify characters typed with the left hand. While poor to average typists often use only one shift key to modify characters on either side of the keyboard. Furthermore, our qualitative observations seemed to indicate that many other user specific shift correlations existed. To allow for the verification of this hypothesis, each user was assigned the following credentials in the registration section:

- Username- in the following format:

Firstname.Lastname

first initial of first and last names capitalized

- Password1- an 8 character control password in the following format: password

these passwords were all english words or names commonly found in cryptographic attack dictionaries [24] 
Examples: computer, swimming, springer

- Password2- a 12 character test password in the following format: SUUDLLLLDUUS

$-\mathrm{S}=$ special symbol

$-\mathrm{U}=$ uppercase letter

$-\mathrm{L}=$ lowercase letter

$-\mathrm{D}=$ digit

these passwords were randomly generated to conform to the format above Examples: +AL4lfav8TB=,_UC8gkum5WH

Figure 4.1 shows the layout of the registration screen in which users are provided with system credentials.

Beyond the input, the behavioral nature of this biometric scheme required a slightly more involved data collection process than what is typical in conventional physiological biometric systems. Most notably, one cannot simply compare genuine input of one user to genuine input from another user in order to establish imposter input or FAR measures as the passwords (at least in the study) are not the same for every individual. Therefore, two sections had to be developed; one in which users input the credentials given to them in the enrollment phase and another in which users input the credentials assigned to other users. In terms of a conventional biometric system, the former section represents genuine data and the 


\section{Username / Password Verification Through Dynamic Keystroke Analysis- Nick Bartlow}

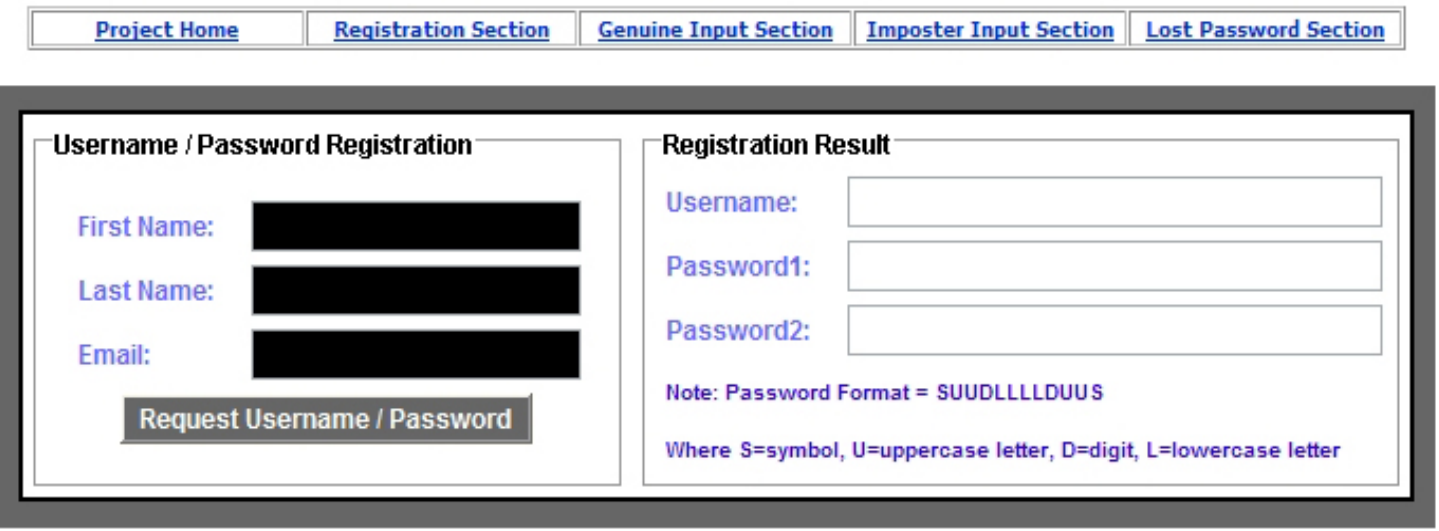

Figure 4.1: System Registration Section

latter represents imposter data. Figure 4.2 shows the relatively simple genuine input screen.

Within this screen, users were asked to input each of their credentials (username + password1 and username + password2) five times each day for approximately 3 weeks.

The imposter input screen was slightly different and can be seen in Figure 4.3. In this section, users were provided with credentials of users other than themselves from the database. In that regard, a user would always select his / her username from the "My UserName" section for the purposes of not inadvertently collecting genuine data in the imposter section. Upon selection of "My UserName," the database would populate the "Imposter Credentials to Input" section in a load-balanced way, selecting a pair of credentials that had a relatively low amount of imposter data collected. At this point the user would simply login in the same manner as in the genuine input section and pending successful input, the credential section 


\section{Username / Password Verification Through Dynamic Keystroke Analysis- Nick Bartlow}

\begin{tabular}{l||l|l||l|l|}
\hline Project Home & Reqistration Section & Genuine Input Section & Imposter Input Section & Lost Password Section \\
\hline
\end{tabular}

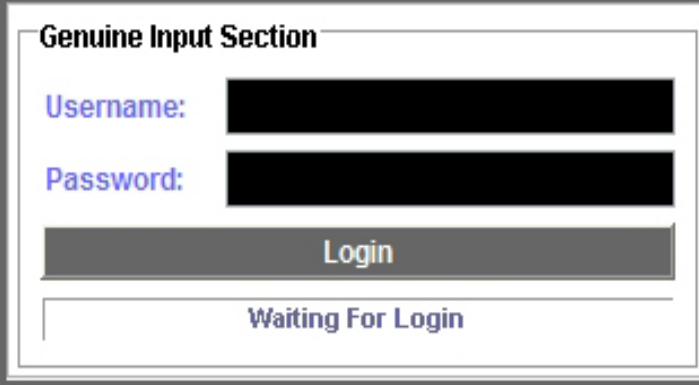

Figure 4.2: Genuine Input Section

\section{Username / Password Verification Through Dynamic Keystroke Analysis- Nick Bartlow}

\begin{tabular}{l|l|l|l|l|} 
Project Home & Reqistration Section & Genuine Input Section & Imposter Input Section & Lost Password Section \\
\hline
\end{tabular}
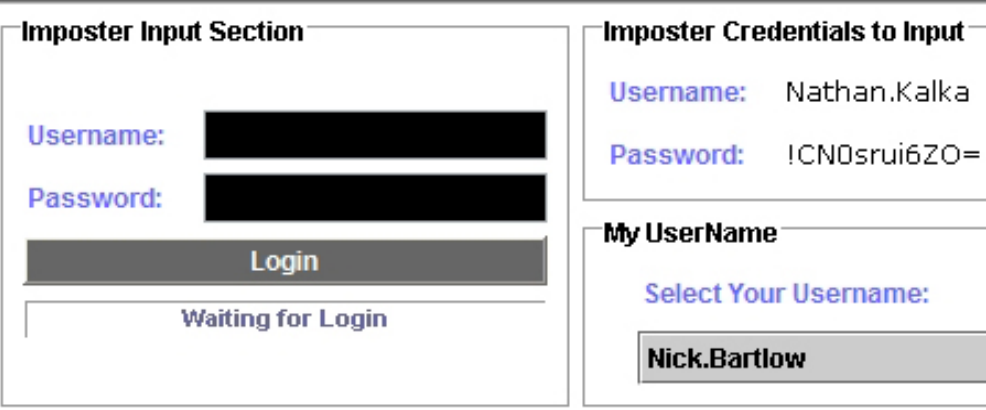

hyy UserName

Select Your Username:

Nick.Bartlow

Figure 4.3: Genuine Input Section 
would repopulate with potentially new credentials. Similar to the genuine input screen, users were asked to input a total of 10 sequences per day for this screen.

Lastly, we look into what was actually collected for each sequence. Using the built-in Java event handlers, key hold times and inter-key delays accurate to the millisecond were collected for each keystroke in both the username and password for each sequence. After some calculation, the following attributes were included to form a feature vector for each input sequence, as seen in Table 4.1. Each attribute is not necessarily completely orthogonal to

$\begin{array}{cllll}1 & \text { USERID } & 22 & \text { RIGHT_SHIFT_HOLD_STD } \\ 2 & \text { TOTAL_STROKES } & 23 & \text { RIGHT_SHIFT_HOLD_MAX } \\ 3 & \text { HOLD_AVG } & 24 & \text { RIGHT_SHIFT_HOLD_MIN } \\ 4 & \text { HOLD_STD } & 25 & \text { RIGHT_SHIFT_HOLD_TOTAL } \\ 5 & \text { HOLD_MAX } & 26 & \text { DELAY_AVG } \\ 6 & \text { HOLD_MIN } & 27 & \text { DELAY_STD } \\ 7 & \text { HOLD_TOTAL } & 28 & \text { DELAY_MAX } \\ 8 & \text { TOTAL_SHIFTS } & 29 & \text { DELAY_MIN } \\ 9 & \text { SHIFT_HOLD_AVG } & 30 & \text { DELAY_TOTAL } \\ 10 & \text { SHIFT_HOLD_STD } & 31 & \text { LEFT_SHIFT_DELAY_AVG } \\ 11 & \text { SHIFT_HOLD_MAX } & 32 & \text { LEFT_SHIFT_DELAY_STD } \\ 12 & \text { SHIFT_HOLD_MIN } & 33 & \text { LEFT_SHIFT_DELAY_MAX } \\ 13 & \text { SHIFT_HOLD_TOTAL } & 34 & \text { LEFT_SHIFT_DELAY_MIN } \\ 14 & \text { LEFT_SHIFTS } & 35 & \text { LEFT_SHIFT_DELAY_TOTAL } \\ 15 & \text { LEFT_SHIFT_HOLD_AVG } & 36 & \text { RIGHT_SHIFT_DELAY_AVG } \\ 16 & \text { LEFT_SHIFT_HOLD_STD } & 37 & \text { RIGHT_SHIFT_DELAY_STD } \\ 17 & \text { LEFT_SHIFT_HOLD_MAX } & 38 & \text { RIGHT_SHIFT_DELAY_MAX } \\ 18 & \text { LEFT_SHIFT_HOLD_MIN } & 39 & \text { RIGHT_SHIFT_DELAY_MIN } \\ 19 & \text { LEFT_SHIFT_HOLD_TOTAL } & 40 & \text { RIGHT_SHIFT_DELAY_TOTAL } \\ 20 & \text { RIGHT_SHIFTS } & 41 & \text { TYPE }\{\text { G=genuine, I=Imposter } \\ 21 & \text { RIGHT_SHIFT_HOLD_AVG } & & \end{array}$

Table 4.1: Feature Vector Collected for Each Input Sequence

every other attribute, but we believed that the inclusion of semi-redundant features may be beneficial in terms of the classification process. Detailed descriptions of these features can be found in Appendix A. Later sections will discuss how the sequences were analyzed. 
Should the results prove acceptable, the system would easily convert to a biometrics-based password hardening system which would have the ability to be deployed without user supervision in enrollment and verification phases. Furthermore, the behavior-based nature of the biometric would allow for replaceability through assignment of a new password.

\subsection{Data Collection Results}

At the time of final analysis the database had a total of 53 users with over 10,000 total input sequences. After applying a minimum cutoff of 15 sequences of each type to be included in the study, a total of 41 users and 8,775 total sequences were used. Out of the 8,775 total sequences, 5,078 were of type genuine and 3,697 were of type imposter. The demographics of the database represent a fairly diverse population in many regards, the gender split was approximately equal, ages ranged from mid-teens to individuals in their late 50's to early 60 's, there was also a relatively diverse racial makeup. Perhaps most importantly, the typing ability of the population was also extremely diverse, ranging from the most inept "hunt and peck" typists to individuals typists with professional training / experience. Due to the nature of recruiting, the collection process lasted approximately one month but is currently still ongoing for the purposes of future work. 


\section{Chapter 5}

\section{Classification / Matching}

The matching block of most biometric systems operates over one or more relatively simple distance metrics used to determine the difference between sample and template feature vectors. Such distance metrics include absolute, Euclidean, Manhattan, Hamming, Mahalanobis, least squares, etc [6][25]. All of these can easily be used when the feature vectors are of the same fixed-length. Some biometrics such as fingerprints often cannot rely on fixedlength feature vectors as the number of uniquely identifying characteristics such as minutiae vary from individual to individual. Furthermore, variation also occurs from sample to sample within the same individual. These biometrics require somewhat more complex matchers that must be capable of comparing feature vectors of unequal size.

Fortunately, the data gathered in this keystroke dynamics study allowed for fixed size feature vectors. That having been said, initial tests employing traditional distance metrics did not yield promising results as they seemed incapable of determining the complexity of feature 
importance over the entire scope of users. Although this does not indicate such a method couldn't achieve high results, we knew other more "promising" methods could be employed instead. As mentioned in the overview, the field of machine learning has various applications; this section outlines the ability of various machine learning algorithms to provide verification of user identity in our study. Performance analysis, measured by overall classification accuracy, FAR, and FRR was conducted on all data sets and tallied for short password sequences, long password sequences, and short and long sequences concatenated.

Once again, the goal of the study was to use the keystroke dynamics biometric to augment the security of a traditional username and password authentication scheme. Although the performance measures can be taken as a biometric system alone, it was not the intended focus of this study. As a result, the traditional frame of thought does not directly apply. The FAR can be thought of as the new penetration rate relative to the $100 \%$ penetration rate associated with a compromised or stolen password. Furthermore, the FRR can be thought of as the decrease in usability of the system due to the occasional misclassification of an authorized user's input. Finally, the overall classification accuracy simply represents the summation of the two types of errors divided by the total number of instances.

\subsection{Machine Learners}

We selected several machine learners for the analysis. The list is significant for a number of reasons. First, it represents a somewhat "classic" list of algorithms used for performance comparison on a given set of data [26]. Second, it includes a wide range of machine learning 
approaches including decision tree, probabilistic, on-line linear separation, and meta-learning methods that use boosting to augment simpler learning algorithms. Third, at least one algorithm can presumably be compared to results of previous studies. Besides performance measures, each section will also include explanation of each learning algorithm. Learning with OneR, NaiveBayes, VotedPerceptron, and LogitBoost was conducted by scripting runs to the command line interface of the Weka machine learning software package [27]. All algorithms from Weka were run using the default Weka parameters for each algorithm, which can be found in Appendix B. Similar techniques were applied with Quinlan's C5.0 proprietary software package [28] and Liaw's freely distributed R [29] implementation [30] of Breiman's Random Forests algorithm [31][32].

\subsubsection{OneR}

The most simplistic learner tested, OneR gets its name from the fact that it generates one-level decision trees [8]. To do so, the algorithm diagnoses each value present for each attribute within the instances being considered. It then generates one-level trees by finding the most frequently occurring class for each of the aforementioned values. The classification error is then calculated for this optimized value-attribute combination. Finally, the rule with the lowest overall error rate is chosen out of the optimized value-attribute combinations [8]. Although this method is extremely intuitive and simple, it performs surprisingly well on many data sets. Table 5.1 shows the performance of OneR in terms of overall classification accuracy, FAR, and FRR. The table represents aggregate results over the 82 data sets acquired (41 users, short password and long password). The blue rows represent sequences involving the eight letter ( $\mathrm{S}=$ short) English passwords and the yellow rows represent the sequences 
involving the twelve character $(\mathrm{L}=\mathrm{long})$, shift-key intensive passwords. Each section has two rows; totals and means. Totals reflect the summation of results for all users in all of the categories. The means represent an average across all users for each measure. As the first

\begin{tabular}{|c|c|c|c|c|c|c|c|c|c|}
\hline $\begin{array}{l}\text { Data } \\
\text { Set }\end{array}$ & $\begin{array}{l}\text { Classif. } \\
\text { Errors }\end{array}$ & $\begin{array}{l}\text { Num } \\
\text { Inst. }\end{array}$ & $\begin{array}{c}\text { Classif. } \\
\text { Acc. }\end{array}$ & $\begin{array}{c}\text { False } \\
\text { Accepts }\end{array}$ & $\begin{array}{l}\text { Num } \\
\text { Imp. } \\
\text { Inst. }\end{array}$ & FAR & $\begin{array}{c}\text { False } \\
\text { Rejects }\end{array}$ & $\begin{array}{l}\text { Num } \\
\text { Gen. } \\
\text { Inst. }\end{array}$ & FRR \\
\hline S Total & 174 & 1451 & 0.880 & 92 & 622 & 0.148 & 82 & 829 & 0.099 \\
\hline S Mean & 4.244 & 35.390 & 0.873 & 2.244 & 15.171 & 0.140 & 2.000 & 20.220 & 0.133 \\
\hline L Total & 134 & 1405 & 0.905 & 77 & 582 & 0.132 & 57 & 823 & 0.069 \\
\hline L Mean & 3.268 & 34.268 & 0.891 & 1.878 & 14.195 & 0.132 & 1.390 & 20.073 & 0.108 \\
\hline
\end{tabular}

Table 5.1: OneR's Overall Performance

set of results presented, OneR clearly shows there is a noticeable difference between genuine and imposter sequences achieving total classification accuracy of $88 \%$ for short password sequences and $90.5 \%$ for long sequences. Given the simplicity of this learner, we believe in the merit of employing machine learning techniques to separate the classes of the keystroke sequences. Despite the classification accuracy, it is important to note the mean FAR and FRR's of $14 \%$ and $13.3 \%$ and $13.2 \%$ and $10.8 \%$ for the two different password types. Given modern standards, this would not be sufficient for a biometric system, yet it may prove sufficient for this particular application of biometrics.

\subsubsection{NaiveBayes}

Having looked at one of the simplest learners, we next studied the performance of a more complex learning scheme. The Naive Bayes learning algorithm is a well known machine learner based on the Bayes's rule of conditional probability as seen in Equation 5.1[33]. Naive Bayes generates class probabilities using the multiplicative rule for multiple events. 


$$
\operatorname{Pr}[H \mid E]=\frac{\operatorname{Pr}[E \mid H] * \operatorname{Pr}[H]}{\operatorname{Pr}[E]}
$$

Although more complex than OneR, Naive Bayes is still a relatively simple learner. Despite the marginal increase algorithmic complexity, Table 5.2 shows a noticeable increase in performance for at least some of the measures considered. The FAR for long passwords, and

\begin{tabular}{|c|c|c|c|c|c|c|c|c|c|}
\hline $\begin{array}{l}\text { Data } \\
\text { Set }\end{array}$ & $\begin{array}{l}\text { Classif. } \\
\text { Errors }\end{array}$ & $\begin{array}{l}\text { Num } \\
\text { Inst. }\end{array}$ & $\begin{array}{c}\text { Classif. } \\
\text { Acc. }\end{array}$ & $\begin{array}{c}\text { False } \\
\text { Accepts }\end{array}$ & $\begin{array}{l}\text { Num } \\
\text { Imp. } \\
\text { Inst. }\end{array}$ & FAR & $\begin{array}{c}\text { False } \\
\text { Rejects }\end{array}$ & $\begin{array}{l}\text { Num } \\
\text { Gen. } \\
\text { Inst. }\end{array}$ & FRR \\
\hline S Total & 150 & 1451 & 0.897 & 103 & 622 & 0.166 & 47 & 829 & 0.057 \\
\hline S Mean & 3.659 & 35.390 & 0.888 & 2.512 & 15.171 & 0.165 & 1.146 & 20.220 & 0.064 \\
\hline L Total & 95 & 1405 & 0.932 & 57 & 582 & 0.098 & 38 & 823 & 0.046 \\
\hline L Mean & 2.317 & 34.268 & 0.926 & 1.390 & 14.195 & 0.098 & 0.927 & 20.073 & 0.046 \\
\hline
\end{tabular}

Table 5.2: NaiveBayes's Overall Performance

the FRR for both short and long passwords all improved in performance over OneR with increases in mean accuracy of $3.4 \%, 6.9 \%$, and $6.2 \%$, respectively. Since NaiveBayes is based on Bayes's rule of conditional probability, it assumes independence between events. This assumption clearly does not hold up on these data sets as some attributes clearly are not independent of one another, i.e., TOTAL_SHIFT_HOLD, LEFT_SHIFT_HOLD, etc It is also important to note the first instance of a trend of increased performance in long password compared to short password sequences for all three performance measures.

\subsubsection{VotedPerceptron}

Once again increasing algorithmic complexity, the VotedPerceptron algorithm uses a series of vector predictors attained through artificial neural networks to attempt to split two classes 
(genuine, imposter) thought to be linearly separable in multi-dimensional space [34][35][36]. This represents the first algorithm that makes use of multiple "options" by way of a voting scheme. The algorithm places weighted voting power on possible linear separators based with weighting based on the "survival" objective function intrinsic to the Perceptron algorithm [34]. Table 5.3 shows the performance of the first voting scheme based algorithm considered. The use of this algorithm demonstrates noted drop offs in performance in all categories

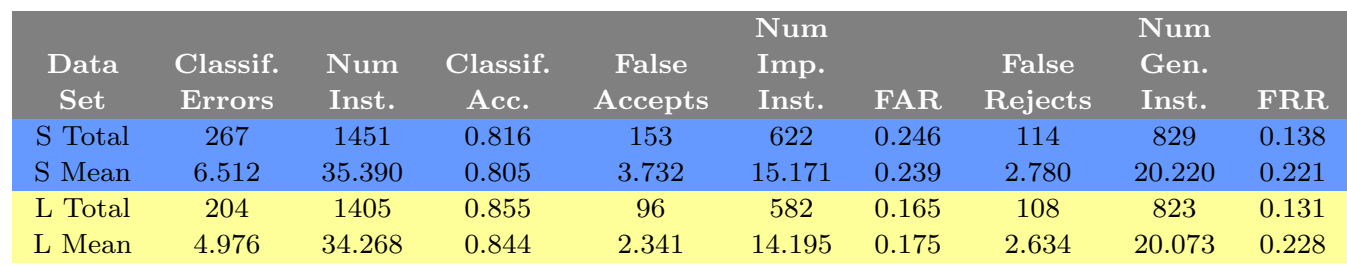

Table 5.3: VotedPerceptron's Overall Performance

relative to OneR and NaiveBayes. At first thought this seems non-intuitive as the casual observer may believe with increased complexity comes increased performance. This premise often does not hold true in machine learning and surface analysis can shed light into why it does not hold true in this case. The perceptron algorithm relies on the linear separability of the genuine and imposter users; the typical two class problem. This may not be the case in our study. Although the classes may be separable, the separation may indeed be non-linear in nature requiring methods such as non-linear regression or cubic splines for splitting.

\subsubsection{LogitBoost}

The LogitBoost machine learning algorithm represents the first "true" boosting algorithm considered to this point. Boosting is the process of combining similar complementary models in the hope that an increase in performance will be achieved. The name boosting comes from 
the fact that model construction is an iterative process with particular incentive placed on defining new models to correct the errors of previous models. Finally, similar to the VotedPerceptron algorithm a weighted voting scheme based on individual model performance is used to construct the overall classification / prediction model. Unlike the VotedPerceptron, LogitBoost is not limited to the use of a single algorithm (perceptron) in which to boost. Many algorithms can be boosted; in our case the simple classification tree algorithm DecisionStump was boosted. Finally, it is important to note that the "Logit" in LogitBoost comes from the fact that it uses additive logistic regression (maximum Bernoulli likelihood as the objective function) as the boosting mechanism [37][8]. Table 5.4 shows the overall performance of the LogitBoost algorithm. On the whole, this learner outperforms all other

\begin{tabular}{cccccccccc} 
& & & & & Num & & \multicolumn{3}{c}{ Num } \\
Data & Classif. & Num & Classif. & False & Imp. & & \multicolumn{2}{c}{ False } & Gen. \\
Set & Errors & Inst. & Acc. & Accepts & Inst. & FAR & Rejects & Inst. & FRR \\
S Total & 97 & 1451 & 0.933 & 51 & 622 & 0.082 & 46 & 829 & 0.055 \\
S Mean & 2.366 & 35.390 & 0.930 & 1.244 & 15.171 & 0.079 & 1.122 & 20.220 & 0.074 \\
L Total & 65 & 1405 & 0.954 & 38 & 582 & 0.065 & 27 & 823 & 0.033 \\
L Mean & 1.585 & 34.268 & 0.951 & 0.927 & 14.195 & 0.062 & 0.659 & 20.073 & 0.038
\end{tabular}

Table 5.4: LogitBoost's Overall Performance

learners considered thus far. It is here that we begin to see FAR (7\% overall mean) and FRR ( $5.6 \%$ overall mean) numbers similar to those attained in previous work. Furthermore, overall classification accuracy begins flirting with the mid to upper $90 \%$ region coming in at 93.3\% and $95.1 \%$ for short and long passwords. Finally, the trend of increased performance in long password sequences vs. short password sequences continues. 


\section{1 .5 C5.0 (See5)}

Quinlan's C5.0 [28] (See5 for Windows) is the successor to his widely used decision tree learner C4.5. Although the source code currently remains proprietary, it is believed that the tree creation techniques have not changed substantially. In brief, the algorithm employs probabilistic methods to grow decision tree branches and then uses a series of techniques to prune sub-trees as to sufficiently generalize the final tree for test purposes (limit overfitting). There are a few noted differences between C4.5 and C5.0, the difference of interest in this paper is the addition of adaptive boosting. Very similar to the ideas described in LogitBoost, when boosting is turned on, C5.0 constructs multiple decision trees instead of only one with the latter trees attempting to alleviate the errors of the previous ones [38]. Once again, a voting scheme is applied to determine the class value for a given instance that has been passed through all the trees generated. Table 5.5 shows the results of the C5.0 decision tree learner with boosting turned on (maximum of 10 boosting iterations). As the figure indicates, C5.0's

\begin{tabular}{cccccccccc} 
Data & Classif. & Num & Classif. & False & $\begin{array}{c}\text { Num } \\
\text { Imp. }\end{array}$ & \multicolumn{3}{c}{ False } & $\begin{array}{c}\text { Gum } \\
\text { Gen. }\end{array}$ \\
Set & Errors & Inst. & Acc. & Accepts & Inst. & FAR & Rejects & Inst. & FRR \\
S Total & 105 & 1451 & 0.928 & 55 & 622 & 0.088 & 50 & 829 & 0.060 \\
S Mean & 2.561 & 35.390 & 0.922 & 1.341 & 15.171 & 0.085 & 1.220 & 20.220 & 0.084 \\
L Total & 81 & 1405 & 0.942 & 42 & 582 & 0.072 & 39 & 823 & 0.047 \\
L Mean & 1.976 & 34.268 & 0.938 & 1.024 & 14.195 & 0.074 & 0.951 & 20.073 & 0.055
\end{tabular}

Table 5.5: C5.0's Overall Performance

performance is good, falling just below that of LogitBoost, the best algorithm thus far. Once again, overall classification accuracy lies in the mid $90 \%$ range. Although, performance is slightly lower than LogitBoost, C5.0 offers one noted advantage in that relatively easy to comprehend "rulesets" can be derived from C5.0's decision trees which might be desired by a system administrator in the event that this was used as matcher in deployed biometric 
system [38].

\subsubsection{Random Forests}

The last machine learning algorithm considered in the study was Breiman and Cutler's Random Forests [31][32]. An elegant and powerful algorithm, Random Forests gets it name for two different reasons. One, it is an algorithm that is based on the development of a "forest" of decision tree classifiers, each classifier being similar to a C5.0 decision tree. Two, the method of generating the forests is based on the random sampling of features in the attribute space. The tree generation algorithm works as follows: each tree is grown based on a random sample selection from $2 / 3$ of the entire instance population. Then nodes, branches, and leaves are generated by continuously choosing the feature the yields the best split of the data based on $\mathrm{m}$ randomly selected features from the feature space. Sub-tree generation continues the furthest extent possible without pruning. Once all trees have been generated, instances are passed through the trees of the forest and a voting process takes place to determine the classification result. There are a number of attractive advantages to this scheme; this study pays particular attention to two of them. One, due to the $2 / 3$ sampling used to train each tree, the remaining $1 / 3$ out-of-bag (OOB) sample is used test performance. Therefore, specific test and train sets do not have to be generated and the whole scope of data can be included. The ability to define varying voting schemes allows for generation forests tailored to specific matching applications. For instance, a 10\%-90\% voting scheme for genuine and imposter classes places particular emphasis on the minimizing the FRR whereas a 90\%-10\% scheme reverses the requirement, focusing attention on the FAR. This mechanism allows for generation of entire ROC curves whereas previous learners necessarily generate only single- 
ton points on said curves. We generated 19 Random Forests, each with a different voting scheme with voting increments of 0.05, between 0.05-0.95 and 0.95-0.05. Furthermore, for each forest 500 trees were generated and default value of $\mathrm{m}$ was used, in this case 6 , which represents $\sqrt{40}$ or the square root of the number of features in the feature space. For the sake of brevity, three voting schemes are shown here; the scheme with the EER minimized (most useful for inter learner comparisons), one focused on the FRR, and the last focused on the FAR. Table C.11 shows the performance of Random Forests using a 0.55-0.45 genuineimposter voting scheme which seemed to minimize the overall EER across all voting schemes. Appendix C contains the same tables for all 19 Random Forests. It can be seen here that

\begin{tabular}{cccccccccc} 
Data & Classif. & Num & Classif. & False & $\begin{array}{c}\text { Num } \\
\text { Imp. }\end{array}$ & \multicolumn{3}{c}{ False } & $\begin{array}{c}\text { Gum. } \\
\text { Gen. }\end{array}$ \\
Set & Errors & Inst. & Acc. & Accepts & Inst. & FAR & Rejects & Inst. & FRR \\
S Total & 230 & 4458 & 0.948 & 109 & 1851 & 0.059 & 121 & 2607 & 0.046 \\
S Mean & 5.610 & 108.732 & 0.946 & 2.659 & 45.146 & 0.059 & 2.951 & 63.585 & 0.063 \\
L Total & 149 & 4317 & 0.965 & 59 & 1846 & 0.032 & 90 & 2471 & 0.036 \\
L Mean & 3.634 & 105.293 & 0.962 & 1.439 & 45.024 & 0.032 & 2.195 & 60.268 & 0.055
\end{tabular}

Table 5.6: Random Forest Overall Performance with 0.55-0.45 Genuine Imposter Voting Scheme

Random Forests yield the highest performance all learners in all categories considered. FAR and FRR drop down to $4.5 \%$ and $5.9 \%$ on average for all input sequences and $3.2 \%$ and $5.5 \%$ for long password sequences. Note once again, the increased performance between short and long password sequences. Although this performance is certainly comparable to most of the previous work on keystroke dynamics as a biometric, an argument could be made that these numbers do not represent performance required by modern day biometric systems. Taking this prospective comment into account Table C.5 demonstrates the ability to increase "user friendliness" by focusing attention on the FRR through a 0.25-0.75 genuine-imposter voting scheme. Here we see a slight decrease in overall classification accuracy while noticing 


\begin{tabular}{cccccccccc} 
& & & & \multicolumn{3}{c}{ Num } & & \multicolumn{3}{c}{ Num } \\
Data & Classif. & Num & Classif. & False & Imp. & \multicolumn{2}{c}{ False } & Gen. \\
Set & Errors & Inst. & Acc. & Accepts & Inst. & FAR & Rejects & Inst. & FRR \\
S Total & 369 & 4458 & 0.917 & 346 & 1851 & 0.187 & 23 & 2607 & 0.009 \\
S Mean & 9.000 & 108.732 & 0.915 & 8.439 & 45.146 & 0.187 & 0.561 & 63.585 & 0.012 \\
L Total & 279 & 4317 & 0.935 & 263 & 1846 & 0.142 & 16 & 2471 & 0.006 \\
L Mean & 6.805 & 105.293 & 0.932 & 6.415 & 45.024 & 0.143 & 0.390 & 60.268 & 0.012
\end{tabular}

Table 5.7: Random Forest Overall Performance with 0.25-0.75 Genuine Imposter Voting Scheme

a significant increase in FAR. In this case FAR performance is sacrificed for usability. The mean FRR of $1.2 \%$ for both short and long password sequences indicates that approximately one individual will be falsely rejected in every 100 attempts. It is not hard to imagine the application of such a scheme in an environment where security is not of paramount importance such as a photo-sharing or map service web sites. Table C.15 considers the alternate situation high security situation displaying the 0.75-0.25 genuine imposter voting scheme. As mentioned, the focus is flipped with the focus placed on security (FAR) at the cost of

\begin{tabular}{|c|c|c|c|c|c|c|c|c|c|}
\hline & & & & & Num & & & Num & \\
\hline Data & Classif. & Num & Classif. & False & Imp. & & False & Gen. & \\
\hline Set & Errors & Inst. & Acc. & Accepts & Inst. & FAR & Rejects & Inst. & FRR \\
\hline S Total & 329 & 4458 & 0.926 & 38 & 1851 & 0.021 & 291 & 2607 & 0.112 \\
\hline S Mean & 8.024 & 108.732 & 0.919 & 0.927 & 45.146 & 0.021 & 7.098 & 63.585 & 0.151 \\
\hline L Total & 249 & 4317 & 0.942 & 18 & 1846 & 0.010 & 231 & 2471 & 0.093 \\
\hline L Mean & 6.073 & 105.293 & 0.935 & 0.439 & 45.024 & 0.010 & 5.634 & 60.268 & 0.140 \\
\hline
\end{tabular}

Table 5.8: Random Forest Overall Performance with 0.75-0.25 Genuine Imposter Voting Scheme usuability (FRR). Now we see penetration rates of approximately 1 to 2 in 100 attempts with the overall mean FAR at $1 \%$ and $2.1 \%$ for long and short password sequences. It is perhaps even easier to imagine situations where this type of scheme could be applied such as online merchandizing and banking applications, perhaps even online government systems.

Overall we can see the many advantages of the Random Forest algorithm. The real life 
applicability in terms of operating at different areas of the ROC curve along with the overall better performance seems to support choosing it over other any of the other algorithms tested. Further analysis, provided in the following chapters should further corroborate this conclusion.

\subsection{Inter-learner Performance Comparison}

Although the performance of the learners has already been compared indirectly, it seems appropriate to provide another comparison of all learners including 95\% confidence intervals on all three performance measures considered. The minimized EER Random Forest is once again chosen for comparison purposes. Table 5.9 shows this comparison. Once again, the

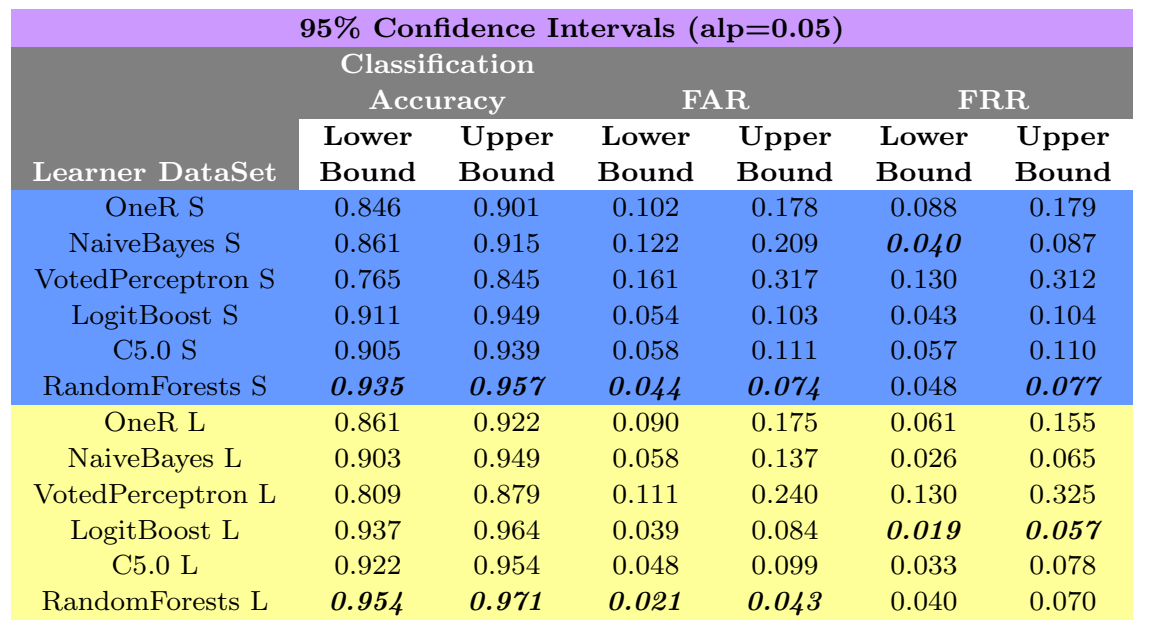

Table 5.9: Learner Performance Comparison Through 95\% Confidence Intervals

short password sequences are indicated by the $\mathrm{S}$ blue rows and the long password sequences are indicated by the L yellow rows. Within the figure, leading performance measures are bolded and italicized. It can be seen that with few exceptions, Random Forests achieves the 
best overall performance in both upper and lower bounds for all three measures. Furthermore, there is only one situation in which a single learner achieves the best overall bound over Random Forests: this is in the case of the LogitBoost relative to the FRR for long password sequences. These results provide rather conclusive evidence that Random Forests are superior in terms of overall performance, FAR, and FRR for the data sets tested especially when one considers that this comparison only considers a single Random Forest voting scheme $(0.55-0.45)$.

\subsection{Short Password Performance vs. Long Password Performance}

Although the comparisons in previous section indicate a clear trend of increased performance from long password sequences compared to short password sequences it is important to look into the statistical significance of the difference. To do so, a Student's t-test was performed on the performances from all 41 users when comparing short to long password sequences. A large difference in performance is indicated by a large $t$ value. Specifically a $t$ value $\geq$ 1.644 indicates a statistical difference for a $95 \%$ confidence level at 80 degrees of freedom (41 short password sets +41 long password sets - 2) [39]. Table 5.10 indicates the results of the test across all learners. The figure indicates that on the whole performance is better in long passwords over short passwords. This lends support to the conclusion that forcing the user to include shift key behavior in passwords helps the classification process in virtually any machine learning algorithm. At the same time only 7 out of 17 (one performance decreased slightly) of these increases can be deemed statistically significant. We hypothesize 


\begin{tabular}{ccccccccccccc} 
& \multicolumn{4}{c}{ Classif. Acc. } & \multicolumn{4}{c}{ FAR } & \multicolumn{1}{c}{ FAR } \\
Learner & Mean S & Mean L & TVal & Sig? & Mean S & Mean L & TVal & Sig? & Mean S & Mean L & TVal & Sig? \\
OneR & 0.873 & 0.891 & 0.995 & No & 0.140 & 0.132 & 0.261 & No & 0.133 & 0.108 & 0.869 & No \\
NaiveBayes & 0.888 & 0.926 & 2.204 & Yes & 0.165 & 0.098 & 2.192 & Yes & 0.064 & 0.046 & 1.430 & No \\
VotedPerceptron & 0.805 & 0.844 & 1.437 & No & 0.239 & 0.175 & 1.247 & No & 0.221 & 0.228 & 0.139 & No \\
LogitBoost & 0.930 & 0.951 & 1.851 & Yes & 0.079 & 0.062 & 1.048 & No & 0.074 & 0.038 & 2.481 & Yes \\
C5.0 & 0.922 & 0.938 & 1.377 & No & 0.085 & 0.074 & 0.613 & No & 0.084 & 0.055 & 1.860 & Yes \\
RandomForests & 0.946 & 0.962 & 3.290 & Yes & 0.059 & 0.032 & 3.251 & Yes & 0.063 & 0.055 & 1.063 & No
\end{tabular}

Table 5.10: Statistical Significance of Difference in Performance Between Short and Long Passwords Across Learners

that this is due to the relatively small tests sets. With such small tests sets, the three measures become very sensitive to classification errors. To consider the effect of sample size in attained performance measures, Table 5.11 shows the statistical significance of our experiments change over the different voting schemes of the Random Forests algorithm. The

\begin{tabular}{|c|c|c|c|c|c|c|c|c|c|c|c|c|}
\hline \multirow[b]{2}{*}{ Learner } & \multicolumn{4}{|c|}{ Classif. Acc. } & \multicolumn{4}{|c|}{ FAR } & \multicolumn{4}{|c|}{ FAR } \\
\hline & Mean S & Mean L & TVal & Sig? & Mean S & Mean L & $T \mathrm{Val}$ & Sig? & Mean S & Mean L & TVal & Sig? \\
\hline RF (0.05-0.95) & 0.784 & 0.783 & 0.115 & No & 0.486 & 0.469 & 0.774 & No & 0.002 & 0.003 & 1.017 & No \\
\hline RF (0.10-0.90) & 0.840 & 0.851 & 1.200 & No & 0.363 & 0.324 & 1.837 & Yes & 0.004 & 0.006 & 0.912 & No \\
\hline RF (0.15-0.85) & 0.873 & 0.890 & 2.319 & Yes & 0.289 & 0.235 & 2.912 & Yes & 0.004 & 0.009 & 1.858 & Yes \\
\hline $\mathrm{RF}(0.20-0.80)$ & 0.896 & 0.914 & 2.761 & Yes & 0.234 & 0.182 & 2.823 & Yes & 0.008 & 0.011 & 0.703 & No \\
\hline $\mathrm{RF}(0.25-0.75)$ & 0.915 & 0.932 & 2.984 & Yes & 0.187 & 0.143 & 2.666 & Yes & 0.012 & 0.012 & 0.160 & No \\
\hline $\mathrm{RF}(0.30-0.70)$ & 0.924 & 0.945 & 3.475 & Yes & 0.159 & 0.109 & 3.121 & Yes & 0.017 & 0.019 & 0.500 & No \\
\hline RF (0.35-0.65) & 0.933 & 0.952 & 3.097 & Yes & 0.133 & 0.092 & 2.778 & Yes & 0.021 & 0.023 & 0.392 & No \\
\hline RF (0.40-0.60) & 0.939 & 0.955 & 3.051 & Yes & 0.106 & 0.076 & 2.378 & Yes & 0.033 & 0.029 & 0.640 & No \\
\hline $\mathrm{RF}(0.45-0.55)$ & 0.944 & 0.958 & 2.705 & Yes & 0.090 & 0.057 & 2.794 & Yes & 0.038 & 0.038 & 0.085 & No \\
\hline $\mathrm{RF}(0.50-0.50)$ & 0.945 & 0.962 & 3.086 & Yes & 0.075 & 0.044 & 2.952 & Yes & 0.050 & 0.043 & 0.855 & No \\
\hline RF (0.55-0.45) & 0.946 & 0.962 & 3.290 & Yes & 0.059 & 0.032 & 3.251 & Yes & 0.063 & 0.055 & 1.063 & No \\
\hline $\mathrm{RF}(0.60-0.40)$ & 0.942 & 0.961 & 3.541 & Yes & 0.046 & 0.023 & 3.551 & Yes & 0.078 & 0.067 & 1.302 & No \\
\hline $\mathrm{RF}(0.65-0.35)$ & 0.937 & 0.955 & 3.683 & Yes & 0.037 & 0.016 & 4.365 & Yes & 0.099 & 0.088 & 1.101 & No \\
\hline RF $(0.70-0.30)$ & 0.929 & 0.943 & 2.297 & Yes & 0.028 & 0.013 & 3.911 & Yes & 0.125 & 0.120 & 0.335 & No \\
\hline $\mathrm{RF}(0.75-0.25)$ & 0.919 & 0.935 & 2.528 & Yes & 0.021 & 0.010 & 3.230 & Yes & 0.151 & 0.140 & 0.655 & No \\
\hline RF (0.80-0.20) & 0.902 & 0.924 & 2.805 & Yes & 0.014 & 0.006 & 2.555 & Yes & 0.189 & 0.171 & 0.929 & No \\
\hline RF (0.85-0.15) & 0.879 & 0.900 & 3.005 & Yes & 0.009 & 0.004 & 1.994 & Yes & 0.243 & 0.224 & 0.982 & No \\
\hline $\mathrm{RF}(0.90-0.10)$ & 0.849 & 0.867 & 2.106 & Yes & 0.004 & 0.003 & 0.505 & No & 0.304 & 0.296 & 0.379 & No \\
\hline RF (0.95-0.05) & 0.789 & 0.819 & 2.714 & Yes & 0.003 & 0.001 & 1.125 & No & 0.418 & 0.397 & 0.799 & No \\
\hline
\end{tabular}

Table 5.11: Statistical Significance of Difference in Performance Between Short and Long Passwords Across Random Forests

results shown in this figure are demonstrably different from those in the previous figure. Here we see that 48 / 57 individual measures represent an increase in performance from short passwords to long passwords. Furthermore, of the 48 increases in accuracy we see that 33 represent statistically significant increases. Therefore, over larger data sets sizes we 
see a rise in statistical significance in the increase in performance associated with longer password sequences from $29.4 \%$ (5/17) to $68.8 \%$ (33/48). It is also important to note where the results are significant; we see noted improvement in the FAR. This implies that the longer more complex passwords that require more intense shift key behavior make the task of system penetration much more difficult for imposters. Furthermore, it appears that the FRR remains somewhat steady between the two different password types with only one voting scheme (0.15-0.85) representing a statistically significant increase in the FRR from short to long passwords. This is encouraging in that the long password does not appear to hinder the usability of the system once users have acclimated themselves to the more complex sequence.

\subsection{The Importance of Shift-Key Features}

Besides the performance comparisons between short and long password sequences, we can also rely on output from the Random Forest classifier to determine whether or not shift-key features are important in classifying sequences. For each Random Forest generated, it is possible to output matrices which summarize the importance of features used in classification. One measure of importance that can be attained is the "mean decrease in accuracy." This measure is a reflection of how an individual feature affects classification of out-of-bag instances [32]. The higher the value, the more important the feature (negative values actually represent a decrease in accuracy; the name is misleading). Table 5.12 shows the top twenty features in terms of this measure of importance across all users at the minimized EER

voting scheme $(0.55-0.45)$ in both types of sequences. Features involving the shift-key are 
highlighted in green. Once again, Appendix A provides a detailed explanation of the what the features. Clearly the shift key measures play a role in classification as they show up in

\begin{tabular}{|c|c|c|c|}
\hline \multicolumn{2}{|l|}{ Short Passwords } & \multicolumn{2}{|l|}{ Long Passwords } \\
\hline & Mean & & Mean \\
\hline & Decrease & & Decrease \\
\hline & in & & in \\
\hline Feature & Accuracy & Feature & Accuracy \\
\hline DELAY_TOTAL & 1.626 & DELAY_AVG & 1.486 \\
\hline DELAY_AVG & 1.544 & DELAY_TOTAL & 1.443 \\
\hline HOLD_AVG & 1.172 & HOLD_TOTAL & 0.985 \\
\hline HOLD_TOTAL & 1.160 & SHIFT_HOLD_TOTAL & 0.958 \\
\hline DELAY_STD & 0.930 & HOLD_AVG & 0.957 \\
\hline SHIFT_HOLD_MIN & 0.899 & RIGHT_SHIFT_DELAY_TOTAL & 0.909 \\
\hline SHIFT_HOLD_AVG & 0.884 & DELAY_STD & 0.894 \\
\hline RIGHT_SHIFT_HOLD_TOTAL & 0.867 & RIGHT_SHIFT_DELAY_MAX & 0.864 \\
\hline LEFT_SHIFT_HOLD_TOTAL & 0.829 & RIGHT_SHIFT_DELAY_AVG & 0.841 \\
\hline HOLD_MIN & 0.824 & RIGHT_SHIFT_HOLD_MIN & 0.840 \\
\hline SHIFT_HOLD_TOTAL & 0.822 & LEFT_SHIFT_HOLD_MIN & 0.837 \\
\hline DELAY_MAX & 0.801 & RIGHT_SHIFT_HOLD_TOTAL & 0.808 \\
\hline HOLD_STD & 0.779 & SHIFT_HOLD_MIN & 0.807 \\
\hline LEFT_SHIFT_HOLD_MIN & 0.769 & LEFT_SHIFT_HOLD_TOTAL & 0.792 \\
\hline RIGHT_SHIFT_HOLD_MIN & 0.766 & SHIFT_HOLD_AVG & 0.760 \\
\hline SHIFT_HOLD_MAX & 0.748 & LEFT_SHIFTS & 0.753 \\
\hline RIGHT_SHIFT_HOLD_AVG & 0.737 & RIGHT_SHIFT_HOLD_AVG & 0.753 \\
\hline LEFT_SHIFT_HOLD_AVG & 0.731 & LEFT_SHIFT_HOLD_AVG & 0.749 \\
\hline HOLD_MAX & 0.726 & LEFT_SHIFT_HOLD_MAX & 0.747 \\
\hline RIGHT_SHIFT_HOLD_MAX & 0.702 & RIGHT_SHIFTS & 0.733 \\
\hline
\end{tabular}

Table 5.12: Feature Importance in Input Sequences

fifteen of the top twenty features in long password sequences and eleven out of twenty in short password sequences. This indicates that the long password sequences designed to force shift key behavior did indeed have an impact on the way sequences were classified relative to the short password sequences. This is further supported by looking at the average mean decrease in accuracy of the shift related attributes in the two types of sequences. Out of the fifteen shift related features in the long password sequences, the average mean decrease in accuracy was 0.810 whereas in 11 shift key attributes in short passwords this value averaged 0.796. These numbers further indicate a difference between the two password types. 


\subsection{User Specific Voting Schemes}

With what has been presented thus far, it is clear that a relationship exists between the voting scheme and the performance measures considered. It should be noted that this trend holds true "on the whole" but is not specific to individual users. Previously, it was suggested that that voting scheme that best minimizes the EER for the performance measures across all users fell at 0.55-0.45 imposter-genuine. Figure 5.1 shows the long password sequence performance graphs for four individuals. The intersection of the FAR and FRR curves indicates the EER for each user. Looking at these intersection points, we see the four users all classified similarly relative to attainable EER's. What is important to note is at which Random Forest voting scheme the intersection takes place. There is a noticeable difference in the optimal voting scheme with respect to minimization of the EER. It appears to range from $0.25-0.75$ through $0.8-0.2$ across the four different users. This implies that a keystroke dynamics biometric system would best be suited if it employs user specific matchers. Many reasons can be put forth to explain this phenomenon, the most obvious of which is the user's typing ability. Although it cannot be ascertained from Figure 5.1, there is a notable difference in the typing ability across the four user data set. However, it does not appear that there is a consistent direct relationship between typing ability (speed) and optimal voting scheme as the mapping is initially unclear and would contain many outliers. Appendix D offers these same graphs for each user with both types of password sequences. 


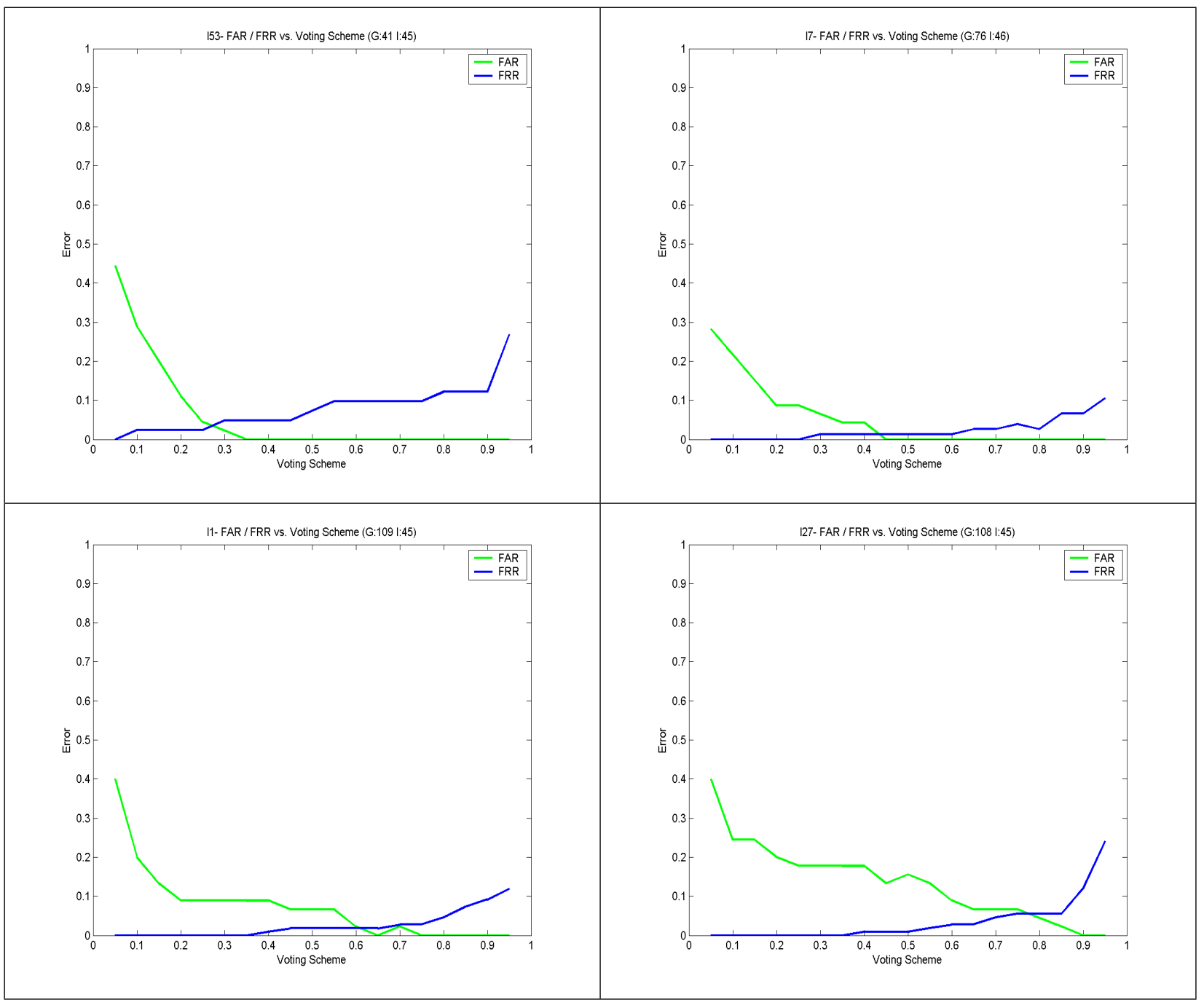

Figure 5.1: Differing RF Voting Schemes for Optimization of EER Across Users

\subsection{Per User System Performance}

The previous section pointed out that users have different optimal voting schemes. Corollary to this fact, the system did exhibit some variability in its ability to classify different users 
given a set voting scheme. Therefore, it would be beneficial to see how the system performed based on the optimal voting scheme relative to the EER for each of the 41 users. Table 5.13 shows this system performance. This shows that the system delivers an EER under $5 \%$ for

\begin{tabular}{cccc}
\multicolumn{4}{c}{ Classification Performance Per User } \\
Password Type & $\mathbf{0 \%} \geq \mathbf{E E R}>\mathbf{5 \%}$ & $\mathbf{5 \%} \geq$ EER $>\mathbf{1 0 \%}$ & EER $>\mathbf{1 0 \%}$ \\
Short & 18 & 17 & 6 \\
Long & 29 & 9 & 3
\end{tabular}

Table 5.13: System Performance Per User

$44 \%(18 / 41)$ of the users with respect to short passwords sequences. With the same $<5 \%$ EER criteria, long password sequences cover $71 \%$ user population (29/41). Once again, this provides more support for the importance of shift-key behavior. If one is willing to accept a $5 \%$ increase in EER, the coverage expands to $85 \%(35 / 41)$ and $93 \%(38 / 41)$ for short and long password sequences. Currently, it is not known what causes the performance of an individual user to rise above $10 \%$. That having been said, there were no individuals for whom the EER was significantly higher than $10 \%$. Appendix D provides sets of graphs for all users of both password types.

\subsection{Overall System Performance}

Combing what has already been done in individual sections, the overall system performance can be measured in a number of ways. Traditionally, biometric systems are measured in terms of a FAR / FRR ROC curve. Figure 5.2 shows this curve for the overall system with respect to mean error rates. This figure illustrates that long passwords have an attainable EER just under $5 \%$ and short passwords fall at approximately $7 \%$. It also shows that the 


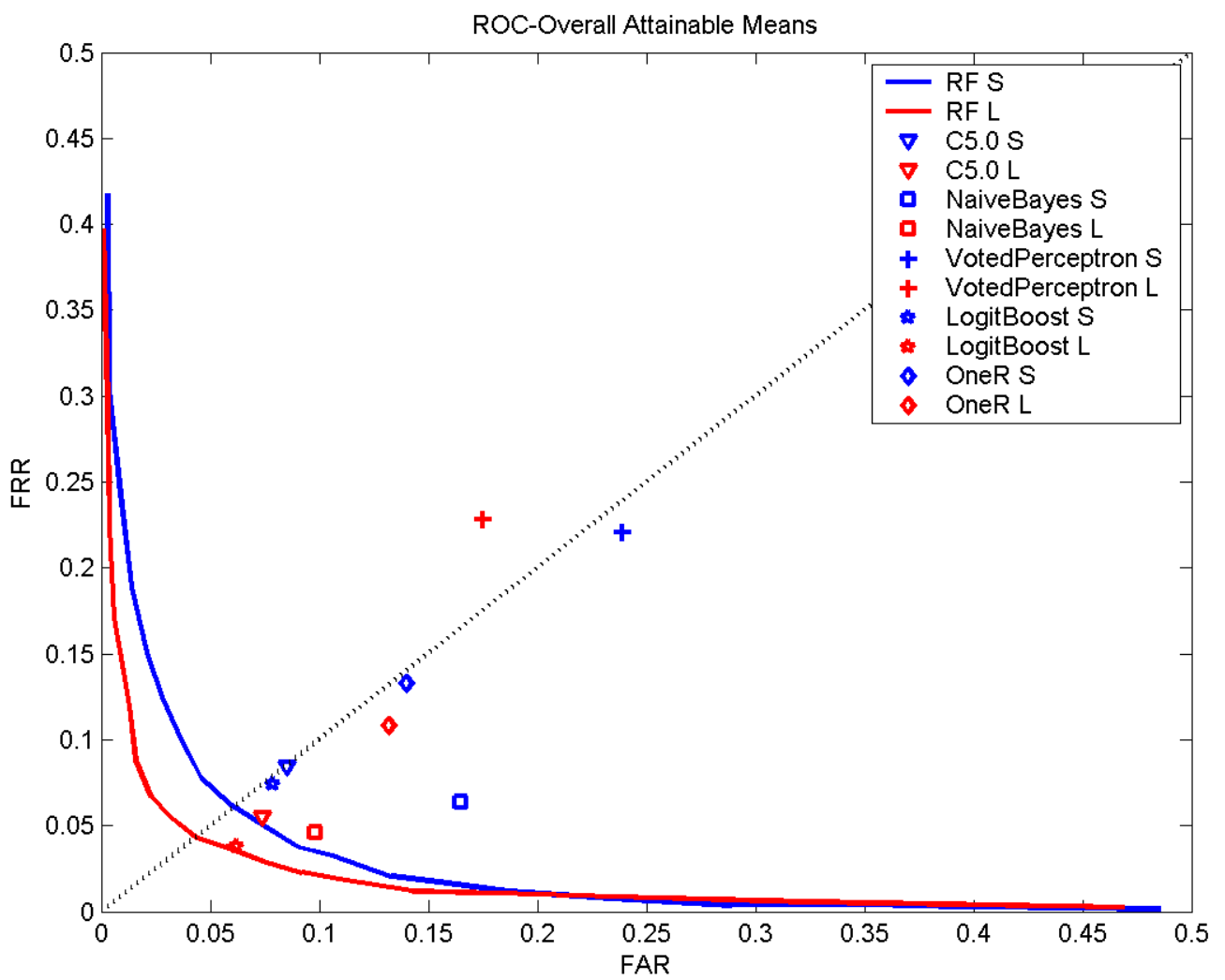

Figure 5.2: Overall System Performance ROC Curve 
only other classifier that can achieve a mean accuracy on par with Random Forests is LogitBoost at FAR and FRR rates of approximately $7 \%$ and $4 \%$ respectively. Table 5.14 shows how this performance relates to password hardening. To consider the ability of our sys-

\begin{tabular}{|ccccc}
\multicolumn{4}{c}{ Password Hardening } \\
FAR(\%) & \multicolumn{2}{c|}{ FRR(\%) } \\
Password Type & Before & After & Before & After \\
Short & 100 & 7 & 0 & 7 \\
\hline Long & 100 & 5 & 0 & 5 \\
\hline
\end{tabular}

Table 5.14: System Password Hardening Effect

tem to serve as a mechanism to increase password based authentication schemes we assume that an imposter attempting unauthorized entry has obtained the targeted user's password. Therefore, it can be assumed that the FAR or penetration rate of the imposter will be $100 \%$. In other words, if imposter knows the targeted users credentials he will always be able to type them in correctly in conventional password based systems. On the other hand, when our keystroke dynamics biometric is used to augment the system, we increase the security in terms of FAR by $93 \%$ and $95 \%$ respective to short and long passwords. These increases are calculated by simply subtracting the keystroke dynamics FAR from the 100\% pentration with out the biometric augment. These significant increases in FAR performance come at a relatively minor price as the associated increases in FRR are 7\% and 5\% in short and long passwords. Therefore, there is only a small sacrifice in usability to achieve a large increase in security. That having been said, one could further tailor this password hardening effect in terms of FAR and FRR to his application specific security needs.

One final look into varying the Random Forests voting scheme solidifies to points that have been supported throughout this chapter. One, complex password sequences involving shift- 


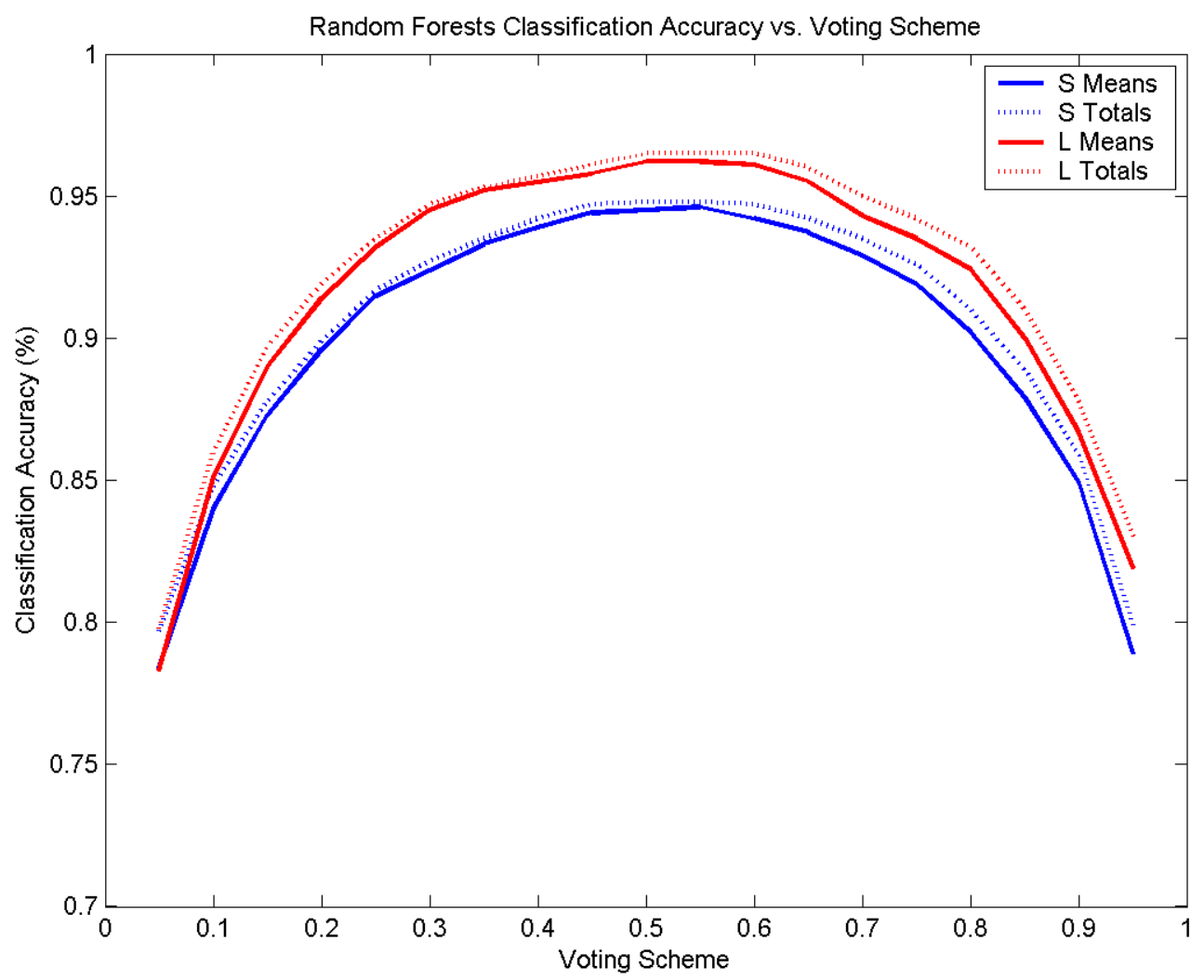

Figure 5.3: Classification Accuracy vs. Random Forests Voting Scheme

key behavior offer a noticeable improvement in performance (classification accuracy, FAR, and EER) over short password sequences that do not require the extensive use of the shift keys. Two, this phenomenon holds true over virtually the entire space of Random Forest voting schemes tested. Figure 5.3 Throughout the course of the symmetrical arch formed by plotting mean classification accuracy versus different Random Forest schemes the long password sequences represented in red remain higher than the short password sequences indicated by the blue curves. 


\section{Chapter 6}

\section{Discussion of Experimental Results}

The results, although encouraging, do not come without caveats. The first notable one is that input was gathered without taking error correction into consideration. This required users to retype their username or password over if the delete or backspace key was hit during an input sequence. Error correction could be added, but it seems it would certainly decrease the accuracy of this keystroke dynamic based user authentication scheme as it relies on such a small input sequence. This hypothesis is supported by the work of Monrose [21].

Related to the previous consideration, we also bring out the difference in user-friendliness between short easy-to-understand passwords compared to somewhat longer much more nonsensical randomly generated passwords that include special characters. This increased security comes with a usability price. The decrease in usability may be justified by the increase in trustworthiness of the authentication scheme. Furthermore, this decrease in usability is arguably lower than that associated with augmentation through a traditional physiological 
biometric scheme as no new hardware is needed and collection of the biometric sample is mostly transparent.

Another item to consider is the way we tested the system. To formulate ROC curves for each individual, imposter data was gathered from all other users in the system. Clearly this could not be easily accomplished in real-world systems. Imposter training data would either have to be synthetically generated (which should be possible) or gathered by the group deploying the system before the system was used. This could be avoided if user specific voting thresholds were not applied but this does not appear to be a feasible alternative.

Intuitively this system can be considered remote and unsupervised. This is an extremely attractive advantage but must be publicized carefully. If the enrollment process is unsupervised, the system is only as good its ability to verify identity through other documentation such as SSN's and the "trusted" information that presumably is only known by the user attempting enrollment. Although this may be a general problem that all authentication schemes face, it is undeniable that human supervision can be used as a countermeasure to decrease fraudulent enrollment in a system.

At a glance the system is taking a "live" biometric in that the user is typing during the course of authentication process. As a result one might consider this characteristic a form of "liveness detection". Unfortunately, the system does not operate in this manner as the client-side, server-side relationship still exists; collection of the keystroke data is independent of server activity until the sequence is completed at which point the feature vector is 
transmitted to the server for matching / classification. Due to this fact, this system remains vulnerable in the same way most biometric systems are; feature vectors could presumably be forged and inserted at various points in the system. The assumed networked nature of the system also renders it vulnerable to traditional network vulnerabilities such a session hijacking and man-in-the-middle attacks [3]. These vulnerabilities are not specific to keystroke dynamics or the proposed password hardening scheme. It simply must be clear that this system does not eliminate these attack points.

Along the same lines, this study did not measure the ability of users to mimic other individuals typing behavior. Due to this fact, our imposter input can be characterized as "zero-effort" attacks. Assuming a talented and properly motivated individual, all behavioral biometric are susceptible to some degree of mimicry. It is not known to what extent our system falls victim to this threat.

As one final consideration, assuming an individual has the ability to type to some degree, the system theoretically does not seem to exude and Failure to Acquire (FTA). In practice however, a small number of laptop keyboard configurations require odd control sequences beyond the use of the shift-keys to generate various a small subset of the special symbols used this study. This fact would require a real life deployment of the system to either rule out such offending special symbols or include other control keys such as CTRL and ALT. Furthermore, an international extension of the this system would have to allow for non-QWERTY keyboards. 



\section{Chapter 7}

\section{Conclusion \& Future Work}

\subsection{Conclusion}

In this study, a web-based application was developed to acquire a large database to be used to establish the viability of keystroke dynamics with usernames and passwords as a possible method of hardening a traditional user authentication scheme. The study was novel in that shift-key behavior was included in the feature matching process. There seemed to be significant performance difference between the eight letter lowercase English passwords and twelve character randomly generated password which required shift-key behavior. The long passwords sequences performed better across numerous performance tests and comparisons providing support for the hypothesis that shift-key behavior plays a significant role in classification, especially in short input circumstances such as username and password. The results may be considered unique in that the scope of the data tested currently outnumbers

what has been tested in previous studies. Performance results would most likely not be 
sufficient for a high security environment as a biometric system alone. That having been said, it seems our algorithms offer an adequate improvement when taken as an unobtrusive holistic approach merging password-based authentication with a behavioral biometric . Furthermore, this system seems to excel where most biometric systems fail in terms of lacking the need of supervision, location independence, decentralization, and replaceability. Based on the results, we believe that users would be able to enroll in a system given proper written instructions; thus, not requiring supervision. Use of the system can take place remotely as opposed to having to travel to an access point. Furthermore, the remote access is not costly in terms of added hardware as it can safely be assumed that all computer users have keyboards. The nature of the system would not require overtly large server infrastructure as the bulk of the processing is down on client computers outside the realm of system responsibility. Finally, keystroke dynamics as a biometric is considered replaceable in the event of compromise. Much like a voice recognition system, the feature template is changed when the password or the passphrase are changed. Individuals are much less likely to be concerned over losing information on how they type versus information about physical characteristics. Also, "the way one types" is not extractable in terms of an adversaries' ability to forcibly aquire it, unlike other physiological biometrics. Although clearly the data set is does not establish the scalability of the final product, it represents a start towards improving the security of password systems that are so common in today's IT based world. 


\subsection{Future Work}

This study could easily spawn more work down the road. It would be interesting to test the system on a large user population, say at the order of tens of thousands to establish scalability. It would also be interesting to determine how easily synthetic data could be generated to supply the classification training process which requires user-specific, password specific data. Along those lines, work could be extended to further customize user specific matching parameters, as suggested by Jain and Ross [40]. Developing an independent measure of a user's ability to acclimate himself to "hard" passwords would help to streamline the enrollment process in an eventual real-world deployment. Further examination into user the specific matching process would be beneficial in terms of performance analysis and possible side effects. For instance, it may be possible to cluster individuals into typing categories much in the same way fingerprints are categorized. Extending that idea leads one to question whether or not username and password keystroke dynamics are feasible for identification modes instead of simply verification. 



\section{Appendix A}

\section{Input Feature Descriptions}

The following is a list of features make up the vector collected as input for each sequence composed of a username and password.

1. USERID- An integer value that uniquely identifies the user from all others in the database.

2. TOTAL_STROKES- An integer value indicating the total number of keystrokes within the sequence.

3. HOLD_AVG- A decimal value indicating the average hold time in milliseconds of all keystrokes within the sequence.

4. HOLD_STD- A decimal value indicating the standard deviation in milliseconds of all keystroke hold times within the sequence.

5. HOLD_MAX-A decimal value indicating the longest time in milliseconds that a key was held down within the sequence.

6. HOLD_MIN- A decimal value indicating the shortest time in milliseconds that a key was held down within the sequence.

7. HOLD_TOTAL- A decimal value indicating the total length of time all keystrokes were held down within the sequence.

8. TOTAL_SHIFTS- An integer value indicating the total number of times either of the shift keys were typed. 
9. SHIFT_HOLD_AVG- A decimal value indicating the average hold time in milliseconds of all shift keystrokes within the sequence.

10. SHIFT_HOLD_STD- A decimal value indicating the standard deviation in milliseconds of all shift keystroke hold times within the sequence.

11. SHIFT_HOLD_MAX-A decimal value indicating the longest time in milliseconds that a shift key was held down within the sequence.

12. SHIFT_HOLD_MIN-A decimal value indicating the shortest time in milliseconds that a shift key was held down within the sequence.

13. SHIFT_HOLD_TOTAL- A decimal value indicating the total length of time all shift key keystrokes were held down within the sequence.

14. LEFT_SHIFTS- An integer value indicating the total number the left shift key was typed within the sequence.

15. LEFT_SHIFT_HOLD_AVG- A decimal value indicating the average hold time in milliseconds of all left shift key keystrokes within the sequence.

16. LEFT_SHIFT_HOLD_STD- A decimal value indicating the standard deviation in milliseconds of all left shift key keystroke hold times within the sequence.

17. LEFT_SHIFT_HOLD_MAX-A decimal value indicating the longest time in milliseconds that the left shift key was held down within the sequence.

18. LEFT_SHIFT_HOLD_MIN- A decimal value indicating the shortest time in milliseconds that the left shift key was held down within the sequence.

19. LEFT_SHIFT_HOLD_TOTAL- A decimal value indicating the total length of time the left shift key was held down within the sequence.

20. RIGHT_SHIFTS- An integer value indicating the total number the left shift key was typed within the sequence.

21. RIGHT_SHIFT_HOLD_AVG-A decimal value indicating the average hold time in milliseconds of all right shift key keystrokes within the sequence.

22. RIGHT_SHIFT_HOLD_STD- A decimal value indicating the standard deviation in milliseconds of all right shift key keystroke hold times within the sequence. 
23. RIGHT_SHIFT_HOLD_MAX- A decimal value indicating the longest time in milliseconds that the right shift key was held down within the sequence.

24. RIGHT_SHIFT_HOLD_MIN- A decimal value indicating the shortest time in milliseconds that the right shift key was held down within the sequence.

25. RIGHT_SHIFT_HOLD_TOTAL- A decimal value indicating the total length of time the left shift key was held down within the sequence.

26. DELAY_AVG- A decimal value indicating the average of the delays between each keystroke pair within the sequence.

27. DELAY_STD- A decimal value indicating the standard deviation of the delays between each keystroke pair within the sequence.

28. DELAY_MAX- A decimal value indicating the longest delay between a keystroke pair within the sequence.

29. DELAY_MIN- A decimal value indicating the shortest delay between a keystroke pair within the sequence.

30. DELAY_TOTAL- A decimal value indicating the total delay between all keystroke pairs within the sequence.

31. LEFT_SHIFT_DELAY_AVG- A decimal value indicating the average of the delays between each keystroke pair involving the left shift key within the sequence.

32. LEFT_SHIFT_DELAY_STD- A decimal value indicating the standard deviation of the delays between each keystroke pair involving the left shift key within the sequence.

33. LEFT_SHIFT_DELAY_MAX-A decimal value indicating the longest of the delays between a keystroke pair involving the left shift key within the sequence.

34. LEFT_SHIFT_DELAY_MIN-A decimal value indicating the shortest of the delays between a keystroke pair involving the left shift key within the sequence.

35. LEFT_SHIFT_DELAY_TOTAL- A decimal value indicating the total delay between all keystroke pairs involving the left shift key within the sequence.

36. RIGHT_SHIFT_DELAY_AVG- A decimal value indicating the average of the delays between each keystroke pair involving the right shift key within the sequence.

37. RIGHT_SHIFT_DELAY_STD- A decimal value indicating the standard deviation of the delays between each keystroke pair involving the right shift key within the sequence. 
38. RIGHT_SHIFT_DELAY_MAX- A decimal value indicating the longest of the delays between a keystroke pair involving the right shift key within the sequence.

39. RIGHT_SHIFT_DELAY_MIN-A decimal value indicating the shortest of the delays between a keystroke pair involving the right shift key within the sequence.

40. RIGHT_SHIFT_DELAY_TOTAL- A decimal value indicating the total delay between all keystroke pairs involving the right shift key within the sequence.

41. $\mathbf{T Y P E} \mathbf{G}=$ genuine, $\mathbf{I}=\mathbf{I m p o s t e r}-\mathrm{A}$ character indicating what class the input sequence falls in.

It should be noted that all values having to do with hold times will necessarily be $\geq 0$. With the exception of standard deviation and delays do not have this requirement as any other feature having to do with delays may have a positive or negative value. 


\section{Appendix B}

\section{Weka Parameters}

The following is a table outlining the default parameters used by the this study when running machine learning algorithms from Weka[27]. 


\begin{tabular}{|c|c|}
\hline \multicolumn{2}{|c|}{ OneR } \\
\hline Parameter & Default Value \\
\hline Minimum Bucket Size & 6 \\
\hline \multicolumn{2}{|c|}{ NaiveBayes } \\
\hline Parameter & Default Value \\
\hline Use Kernel Estimator & False \\
\hline User Supervised Discretization & False \\
\hline \multicolumn{2}{|c|}{ VotedPerceptron } \\
\hline Parameter & Default Value \\
\hline Exponent & 1 \\
\hline $\operatorname{maxK}$ & 10000 \\
\hline numIterations & 1 \\
\hline seed & 1 \\
\hline \multicolumn{2}{|c|}{ LogitBoost } \\
\hline Parameter & Default Value \\
\hline Classifier & DecisionStump \\
\hline likelihoodThreshold & $-1.7976931348623157 \mathrm{E} 308$ \\
\hline numFolds & 0 \\
\hline numIterations & 10 \\
\hline numRuns & 1 \\
\hline seed & 1 \\
\hline shrinkage & 1 \\
\hline useResampling & False \\
\hline weightThreshold & 100 \\
\hline
\end{tabular}




\section{Appendix C}

\section{Random Forest Complete Voting}

\section{Scheme Results}

The following set of tables represent the complete results for the 19 Random Forest voting schemes tested in our study.

\begin{tabular}{|c|c|c|c|c|c|c|c|c|c|}
\hline & & & & & Num & & & Num & \\
\hline Data & Classif. & Num & Classif. & False & Imp. & & False & Gen. & \\
\hline Set & Errors & Inst. & Acc. & Accepts & Inst. & FAR & Rejects & Inst. & FRR \\
\hline S Total & 903 & 4458 & 0.797 & 901 & 1851 & 0.487 & 2 & 2607 & 0.001 \\
\hline S Mean & 22.024 & 108.732 & 0.784 & 21.976 & 45.146 & 0.486 & 0.049 & 63.585 & 0.002 \\
\hline L Total & 870 & 4317 & 0.798 & 865 & 1846 & 0.469 & 5 & 2471 & 0.002 \\
\hline L Mean & 21.220 & 105.293 & 0.783 & 21.098 & 45.024 & 0.469 & 0.122 & 60.268 & 0.003 \\
\hline
\end{tabular}

Table C.1: Random Forest Overall Performance with 0.05-0.95 Genuine Imposter Voting Scheme

\begin{tabular}{|c|c|c|c|c|c|c|c|c|c|}
\hline & & & & & Num & & & Num & \\
\hline Data & Classif. & Num & Classif. & False & Imp. & & False & Gen. & \\
\hline Set & Errors & Inst. & Acc. & Accepts & Inst. & FAR & Rejects & Inst. & FRR \\
\hline S Total & 679 & 4458 & 0.848 & 673 & 1851 & 0.364 & 6 & 2607 & 0.002 \\
\hline S Mean & 16.561 & 108.732 & 0.840 & 16.415 & 45.146 & 0.363 & 0.146 & 63.585 & 0.004 \\
\hline L Total & 605 & 4317 & 0.860 & 597 & 1846 & 0.323 & 8 & 2471 & 0.003 \\
\hline L Mean & 14.756 & 105.293 & 0.851 & 14.561 & 45.024 & 0.324 & 0.195 & 60.268 & 0.006 \\
\hline
\end{tabular}

Table C.2: Random Forest Overall Performance with 0.10-0.90 Genuine Imposter Voting Scheme 


\begin{tabular}{cccccccccc} 
& & & & \multicolumn{3}{c}{ Num } & & \multicolumn{3}{c}{ Num } \\
Data & Classif. & Num & Classif. & False & Imp. & \multicolumn{3}{c}{ False } & Gen. \\
Set & Errors & Inst. & Acc. & Accepts & Inst. & FAR & Rejects & Inst. & FRR \\
S Total & 543 & 4458 & 0.878 & 535 & 1851 & 0.289 & 8 & 2607 & 0.003 \\
S Mean & 13.244 & 108.732 & 0.873 & 13.049 & 45.146 & 0.289 & 0.195 & 63.585 & 0.004 \\
L Total & 445 & 4317 & 0.897 & 434 & 1846 & 0.235 & 11 & 2471 & 0.004 \\
L Mean & 10.854 & 105.293 & 0.890 & 10.585 & 45.024 & 0.235 & 0.268 & 60.268 & 0.009
\end{tabular}

Table C.3: Random Forest Overall Performance with 0.15-0.85 Genuine Imposter Voting Scheme

\begin{tabular}{|c|c|c|c|c|c|c|c|c|c|}
\hline $\begin{array}{l}\text { Data } \\
\text { Set }\end{array}$ & $\begin{array}{l}\text { Classif. } \\
\text { Errors }\end{array}$ & $\begin{array}{l}\text { Num } \\
\text { Inst. }\end{array}$ & $\begin{array}{c}\text { Classif. } \\
\text { Acc. }\end{array}$ & $\begin{array}{c}\text { False } \\
\text { Accepts }\end{array}$ & $\begin{array}{l}\text { Num } \\
\text { Imp. } \\
\text { Inst. }\end{array}$ & FAR & $\begin{array}{c}\text { False } \\
\text { Rejects }\end{array}$ & $\begin{array}{l}\text { Num } \\
\text { Gen. } \\
\text { Inst. }\end{array}$ & FRR \\
\hline S Total & 449 & 4458 & 0.899 & 434 & 1851 & 0.234 & 15 & 2607 & 0.006 \\
\hline S Mean & 10.951 & 108.732 & 0.896 & 10.585 & 45.146 & 0.234 & 0.366 & 63.585 & 0.008 \\
\hline L Total & 349 & 4317 & 0.919 & 335 & 1846 & 0.181 & 14 & 2471 & 0.006 \\
\hline L Mean & 8.512 & 105.293 & 0.914 & 8.171 & 45.024 & 0.182 & 0.341 & 60.268 & 0.011 \\
\hline
\end{tabular}

Table C.4: Random Forest Overall Performance with 0.20-0.80 Genuine Imposter Voting Scheme

\begin{tabular}{|c|c|c|c|c|c|c|c|c|c|}
\hline $\begin{array}{l}\text { Data } \\
\text { Set }\end{array}$ & $\begin{array}{l}\text { Classif. } \\
\text { Errors }\end{array}$ & $\begin{array}{l}\text { Num } \\
\text { Inst. }\end{array}$ & $\begin{array}{c}\text { Classif. } \\
\text { Acc. }\end{array}$ & $\begin{array}{c}\text { False } \\
\text { Accepts }\end{array}$ & $\begin{array}{l}\text { Num } \\
\text { Imp. } \\
\text { Inst. }\end{array}$ & FAR & $\begin{array}{c}\text { False } \\
\text { Rejects }\end{array}$ & $\begin{array}{l}\text { Num } \\
\text { Gen. } \\
\text { Inst. }\end{array}$ & FRR \\
\hline S Total & 369 & 4458 & 0.917 & 346 & 1851 & 0.187 & 23 & 2607 & 0.009 \\
\hline S Mean & 9.000 & 108.732 & 0.915 & 8.439 & 45.146 & 0.187 & 0.561 & 63.585 & 0.012 \\
\hline L Total & 279 & 4317 & 0.935 & 263 & 1846 & 0.142 & 16 & 2471 & 0.006 \\
\hline L Mean & 6.805 & 105.293 & 0.932 & 6.415 & 45.024 & 0.143 & 0.390 & 60.268 & 0.012 \\
\hline
\end{tabular}

Table C.5: Random Forest Overall Performance with 0.25-0.75 Genuine Imposter Voting Scheme

\begin{tabular}{|c|c|c|c|c|c|c|c|c|c|}
\hline $\begin{array}{l}\text { Data } \\
\text { Set }\end{array}$ & $\begin{array}{l}\text { Classif. } \\
\text { Errors }\end{array}$ & $\begin{array}{l}\text { Num } \\
\text { Inst. }\end{array}$ & $\begin{array}{c}\text { Classif. } \\
\text { Acc. }\end{array}$ & $\begin{array}{c}\text { False } \\
\text { Accepts }\end{array}$ & $\begin{array}{l}\text { Num } \\
\text { Imp. } \\
\text { Inst. }\end{array}$ & FAR & $\begin{array}{c}\text { False } \\
\text { Rejects }\end{array}$ & $\begin{array}{l}\text { Num } \\
\text { Gen. } \\
\text { Inst. }\end{array}$ & FRR \\
\hline S Total & 327 & 4458 & 0.927 & 295 & 1851 & 0.159 & 32 & 2607 & 0.012 \\
\hline S Mean & 7.976 & 108.732 & 0.924 & 7.195 & 45.146 & 0.159 & 0.780 & 63.585 & 0.017 \\
\hline L Total & 227 & 4317 & 0.947 & 201 & 1846 & 0.109 & 26 & 2471 & 0.011 \\
\hline L Mean & 5.537 & 105.293 & 0.945 & 4.902 & 45.024 & 0.109 & 0.634 & 60.268 & 0.019 \\
\hline
\end{tabular}

Table C.6: Random Forest Overall Performance with 0.30-0.70 Genuine Imposter Voting Scheme 


\begin{tabular}{cccccccccc} 
& & & & \multicolumn{3}{c}{ Num } & \multicolumn{3}{c}{ Num } \\
Data & Classif. & Num & Classif. & False & Imp. & \multicolumn{2}{c}{ False } & Gen. \\
Set & Errors & Inst. & Acc. & Accepts & Inst. & FAR & Rejects & Inst. & FRR \\
S Total & 288 & 4458 & 0.935 & 247 & 1851 & 0.133 & 41 & 2607 & 0.016 \\
S Mean & 7.024 & 108.732 & 0.933 & 6.024 & 45.146 & 0.133 & 1.000 & 63.585 & 0.021 \\
L Total & 201 & 4317 & 0.953 & 169 & 1846 & 0.092 & 32 & 2471 & 0.013 \\
L Mean & 4.902 & 105.293 & 0.952 & 4.122 & 45.024 & 0.092 & 0.780 & 60.268 & 0.023
\end{tabular}

Table C.7: Random Forest Overall Performance with 0.35-0.65 Genuine Imposter Voting Scheme

\begin{tabular}{cccccccccc} 
Data & Classif. & Num & Classif. & False & $\begin{array}{c}\text { Num } \\
\text { Imp. }\end{array}$ & \multicolumn{3}{c}{ False } & $\begin{array}{c}\text { Gum. } \\
\text { Gen. }\end{array}$ \\
Set & Errors & Inst. & Acc. & Accepts & Inst. & FAR & Rejects & Inst. & FRR \\
S Total & 257 & 4458 & 0.942 & 197 & 1851 & 0.106 & 60 & 2607 & 0.023 \\
S Mean & 6.268 & 108.732 & 0.939 & 4.805 & 45.146 & 0.106 & 1.463 & 63.585 & 0.033 \\
L Total & 186 & 4317 & 0.957 & 141 & 1846 & 0.076 & 45 & 2471 & 0.018 \\
L Mean & 4.537 & 105.293 & 0.955 & 3.439 & 45.024 & 0.076 & 1.098 & 60.268 & 0.029
\end{tabular}

Table C.8: Random Forest Overall Performance with 0.40-0.60 Genuine Imposter Voting Scheme

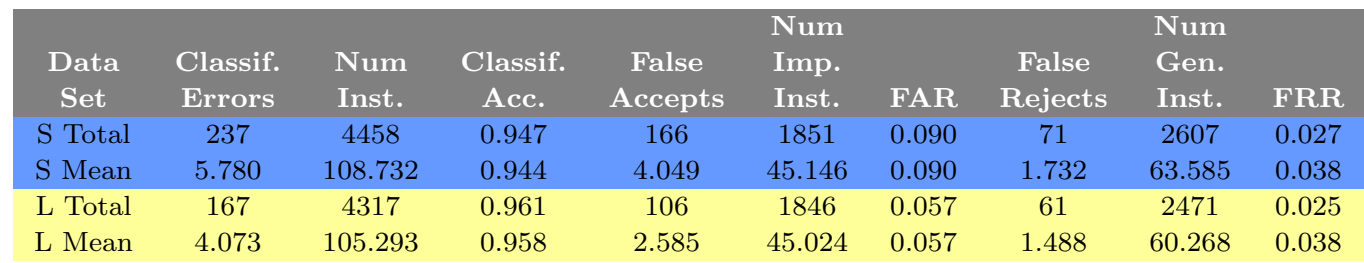

Table C.9: Random Forest Overall Performance with 0.45-0.55 Genuine Imposter Voting Scheme 


\begin{tabular}{|c|c|c|c|c|c|c|c|c|c|}
\hline Data & Classif. & Num & Classif. & False & $\begin{array}{l}\text { Num } \\
\text { Imp. }\end{array}$ & & False & $\begin{array}{l}\text { Num } \\
\text { Gen. }\end{array}$ & \\
\hline Set & Errors & Inst. & Acc. & Accepts & Inst. & FAR & Rejects & Inst. & FRR \\
\hline S Total & 230 & 4458 & 0.948 & 138 & 1851 & 0.075 & 92 & 2607 & 0.035 \\
\hline S Mean & 5.610 & 108.732 & 0.945 & 3.366 & 45.146 & 0.075 & 2.244 & 63.585 & 0.050 \\
\hline L Total & 153 & 4317 & 0.965 & 81 & 1846 & 0.044 & 72 & 2471 & 0.029 \\
\hline L Mean & 3.732 & 105.293 & 0.962 & 1.976 & 45.024 & 0.044 & 1.756 & 60.268 & 0.043 \\
\hline
\end{tabular}

Table C.10: Random Forest Overall Performance with 0.50-0.50 Genuine Imposter Voting Scheme

\begin{tabular}{cccccccccc} 
Data & Classif. & Num & Classif. & False & $\begin{array}{c}\text { Num } \\
\text { Imp. }\end{array}$ & \multicolumn{3}{c}{ False } & $\begin{array}{c}\text { Gum } \\
\text { Gen. }\end{array}$ \\
Errors & Inst. & Acc. & Accepts & Inst. & FAR & Rejects & Inst. & FRR \\
S Total & 230 & 4458 & 0.948 & 109 & 1851 & 0.059 & 121 & 2607 & 0.046 \\
S Mean & 5.610 & 108.732 & 0.946 & 2.659 & 45.146 & 0.059 & 2.951 & 63.585 & 0.063 \\
L Total & 149 & 4317 & 0.965 & 59 & 1846 & 0.032 & 90 & 2471 & 0.036 \\
L Mean & 3.634 & 105.293 & 0.962 & 1.439 & 45.024 & 0.032 & 2.195 & 60.268 & 0.055
\end{tabular}

Table C.11: Random Forest Overall Performance with 0.55-0.45 Genuine Imposter Voting Scheme

$\begin{array}{cccccccccc}\text { Data } & \text { Classif. } & \text { Num } & \text { Classif. } & \text { False } & \begin{array}{c}\text { Nump. } \\ \text { Imp. }\end{array} & \text { False } & \begin{array}{c}\text { Gum } \\ \text { Gen. }\end{array} \\ \text { Set } & \text { Errors } & \text { Inst. } & \text { Acc. } & \text { Accepts } & \text { Inst. } & \text { FAR } & \text { Rejects } & \text { Inst. } & \text { FRR } \\ \text { S Total } & 238 & 4458 & 0.947 & 86 & 1851 & 0.046 & 152 & 2607 & 0.058 \\ \text { S Mean } & 5.805 & 108.732 & 0.942 & 2.098 & 45.146 & 0.046 & 3.707 & 63.585 & 0.078 \\ \text { L Total } & 151 & 4317 & 0.965 & 43 & 1846 & 0.023 & 108 & 2471 & 0.044 \\ \text { L Mean } & 3.683 & 105.293 & 0.961 & 1.049 & 45.024 & 0.023 & 2.634 & 60.268 & 0.067\end{array}$

Table C.12: Random Forest Overall Performance with 0.60-0.40 Genuine Imposter Voting Scheme 


\begin{tabular}{cccccccccc} 
& & & & \multicolumn{3}{c}{ Num } & \multicolumn{3}{c}{ Num } \\
Data & Classif. & Num & Classif. & False & Imp. & \multicolumn{3}{c}{ False } & Gen. \\
Set & Errors & Inst. & Acc. & Accepts & Inst. & FAR & Rejects & Inst. & FRR \\
S Total & 260 & 4458 & 0.942 & 68 & 1851 & 0.037 & 192 & 2607 & 0.074 \\
S Mean & 6.341 & 108.732 & 0.937 & 1.659 & 45.146 & 0.037 & 4.683 & 63.585 & 0.099 \\
L Total & 171 & 4317 & 0.960 & 29 & 1846 & 0.016 & 142 & 2471 & 0.057 \\
L Mean & 4.171 & 105.293 & 0.955 & 0.707 & 45.024 & 0.016 & 3.463 & 60.268 & 0.088
\end{tabular}

Table C.13: Random Forest Overall Performance with 0.65-0.35 Genuine Imposter Voting Scheme

\begin{tabular}{|c|c|c|c|c|c|c|c|c|c|}
\hline $\begin{array}{l}\text { Data } \\
\text { Set }\end{array}$ & $\begin{array}{l}\text { Classif. } \\
\text { Errors }\end{array}$ & $\begin{array}{l}\text { Num } \\
\text { Inst. }\end{array}$ & $\begin{array}{c}\text { Classif. } \\
\text { Acc. }\end{array}$ & $\begin{array}{c}\text { False } \\
\text { Accepts }\end{array}$ & $\begin{array}{l}\text { Num } \\
\text { Imp. } \\
\text { Inst. }\end{array}$ & FAR & $\begin{array}{c}\text { False } \\
\text { Rejects }\end{array}$ & $\begin{array}{l}\text { Num } \\
\text { Gen. } \\
\text { Inst. }\end{array}$ & FRR \\
\hline S Total & 291 & 4458 & 0.935 & 52 & 1851 & 0.028 & 239 & 2607 & 0.092 \\
\hline S Mean & 7.098 & 108.732 & 0.929 & 1.268 & 45.146 & 0.028 & 5.829 & 63.585 & 0.125 \\
\hline L Total & 215 & 4317 & 0.950 & 24 & 1846 & 0.013 & 191 & 2471 & 0.077 \\
\hline L Mean & 5.244 & 105.293 & 0.943 & 0.585 & 45.024 & 0.013 & 4.659 & 60.268 & 0.120 \\
\hline
\end{tabular}

Table C.14: Random Forest Overall Performance with 0.70-0.30 Genuine Imposter Voting Scheme

\begin{tabular}{|c|c|c|c|c|c|c|c|c|c|}
\hline & & & & & Num & & & Num & \\
\hline Data & Classif. & Num & Classif. & False & Imp. & & False & Gen. & \\
\hline Set & Errors & Inst. & Acc. & Accepts & Inst. & FAR & Rejects & Inst. & FRR \\
\hline S Total & 329 & 4458 & 0.926 & 38 & 1851 & 0.021 & 291 & 2607 & 0.112 \\
\hline S Mean & 8.024 & 108.732 & 0.919 & 0.927 & 45.146 & 0.021 & 7.098 & 63.585 & 0.151 \\
\hline L Total & 249 & 4317 & 0.942 & 18 & 1846 & 0.010 & 231 & 2471 & 0.093 \\
\hline L Mean & 6.073 & 105.293 & 0.935 & 0.439 & 45.024 & 0.010 & 5.634 & 60.268 & 0.140 \\
\hline
\end{tabular}

Table C.15: Random Forest Overall Performance with 0.75-0.25 Genuine Imposter Voting Scheme

\begin{tabular}{|c|c|c|c|c|c|c|c|c|c|}
\hline $\begin{array}{l}\text { Data } \\
\text { Set }\end{array}$ & $\begin{array}{l}\text { Classif. } \\
\text { Errors }\end{array}$ & $\begin{array}{l}\text { Num } \\
\text { Inst. }\end{array}$ & $\begin{array}{c}\text { Classif. } \\
\text { Acc. }\end{array}$ & $\begin{array}{c}\text { False } \\
\text { Accepts }\end{array}$ & $\begin{array}{l}\text { Num } \\
\text { Imp. } \\
\text { Inst. }\end{array}$ & FAR & $\begin{array}{c}\text { False } \\
\text { Rejects }\end{array}$ & $\begin{array}{l}\text { Num } \\
\text { Gen. } \\
\text { Inst. }\end{array}$ & FRR \\
\hline S Total & 403 & 4458 & 0.910 & 26 & 1851 & 0.014 & 377 & 2607 & 0.145 \\
\hline S Mean & 9.829 & 108.732 & 0.902 & 0.634 & 45.146 & 0.014 & 9.195 & 63.585 & 0.189 \\
\hline L Total & 294 & 4317 & 0.932 & 11 & 1846 & 0.006 & 283 & 2471 & 0.115 \\
\hline L Mean & 7.171 & 105.293 & 0.924 & 0.268 & 45.024 & 0.006 & 6.902 & 60.268 & 0.171 \\
\hline
\end{tabular}

Table C.16: Random Forest Overall Performance with 0.80-0.20 Genuine Imposter Voting Scheme 


\begin{tabular}{|c|c|c|c|c|c|c|c|c|c|}
\hline Data & Classif. & Num & Classif. & False & $\begin{array}{l}\text { Num } \\
\text { Imp. }\end{array}$ & & False & $\begin{array}{l}\text { Num } \\
\text { Gen. }\end{array}$ & \\
\hline Set & Errors & Inst. & Acc. & Accepts & Inst. & FAR & Rejects & Inst. & FRR \\
\hline S Total & 494 & 4458 & 0.889 & 17 & 1851 & 0.009 & 477 & 2607 & 0.183 \\
\hline S Mean & 12.049 & 108.732 & 0.879 & 0.415 & 45.146 & 0.009 & 11.634 & 63.585 & 0.243 \\
\hline L Total & 389 & 4317 & 0.910 & 8 & 1846 & 0.004 & 381 & 2471 & 0.154 \\
\hline L Mean & 9.488 & 105.293 & 0.900 & 0.195 & 45.024 & 0.004 & 9.293 & 60.268 & 0.224 \\
\hline
\end{tabular}

Table C.17: Random Forest Overall Performance with 0.85-0.15 Genuine Imposter Voting Scheme

\begin{tabular}{|c|c|c|c|c|c|c|c|c|c|}
\hline $\begin{array}{l}\text { Data } \\
\text { Set }\end{array}$ & $\begin{array}{l}\text { Classif. } \\
\text { Errors }\end{array}$ & $\begin{array}{l}\text { Num } \\
\text { Inst. }\end{array}$ & $\begin{array}{c}\text { Classif. } \\
\text { Acc. }\end{array}$ & $\begin{array}{c}\text { False } \\
\text { Accepts }\end{array}$ & $\begin{array}{l}\text { Num } \\
\text { Imp. } \\
\text { Inst. }\end{array}$ & FAR & $\begin{array}{c}\text { False } \\
\text { Rejects }\end{array}$ & $\begin{array}{l}\text { Num } \\
\text { Gen. } \\
\text { Inst. }\end{array}$ & FRR \\
\hline S Total & 628 & 4458 & 0.859 & 8 & 1851 & 0.004 & 620 & 2607 & 0.238 \\
\hline S Mean & 15.317 & 108.732 & 0.849 & 0.195 & 45.146 & 0.004 & 15.122 & 63.585 & 0.304 \\
\hline L Total & 525 & 4317 & 0.878 & 6 & 1846 & 0.003 & 519 & 2471 & 0.210 \\
\hline L Mean & 12.805 & 105.293 & 0.867 & 0.146 & 45.024 & 0.003 & 12.659 & 60.268 & 0.296 \\
\hline
\end{tabular}

Table C.18: Random Forest Overall Performance with 0.90-0.10 Genuine Imposter Voting Scheme

\begin{tabular}{|c|c|c|c|c|c|c|c|c|c|}
\hline $\begin{array}{l}\text { Data } \\
\text { Set }\end{array}$ & $\begin{array}{l}\text { Classif. } \\
\text { Errors }\end{array}$ & $\begin{array}{l}\text { Num } \\
\text { Inst. }\end{array}$ & $\begin{array}{c}\text { Classif. } \\
\text { Acc. }\end{array}$ & $\begin{array}{c}\text { False } \\
\text { Accepts }\end{array}$ & $\begin{array}{l}\text { Num } \\
\text { Imp. } \\
\text { Inst. }\end{array}$ & FAR & $\begin{array}{c}\text { False } \\
\text { Rejects }\end{array}$ & $\begin{array}{l}\text { Num } \\
\text { Gen. } \\
\text { Inst. }\end{array}$ & FRR \\
\hline S Total & 896 & 4458 & 0.799 & 5 & 1851 & 0.003 & 891 & 2607 & 0.342 \\
\hline S Mean & 21.854 & 108.732 & 0.789 & 0.122 & 45.146 & 0.003 & 21.732 & 63.585 & 0.418 \\
\hline L Total & 734 & 4317 & 0.830 & 2 & 1846 & 0.001 & 732 & 2471 & 0.296 \\
\hline L Mean & 17.902 & 105.293 & 0.819 & 0.049 & 45.024 & 0.001 & 17.854 & 60.268 & 0.397 \\
\hline
\end{tabular}

Table C.19: Random Forest Overall Performance with 0.95-0.05 Genuine Imposter Voting Scheme 


\section{Appendix D}

\section{User Graphs}

The following section provides all user graphs for both short and long password sequence types. The titles are labeled by password type and userid. Each user has four graphs. 


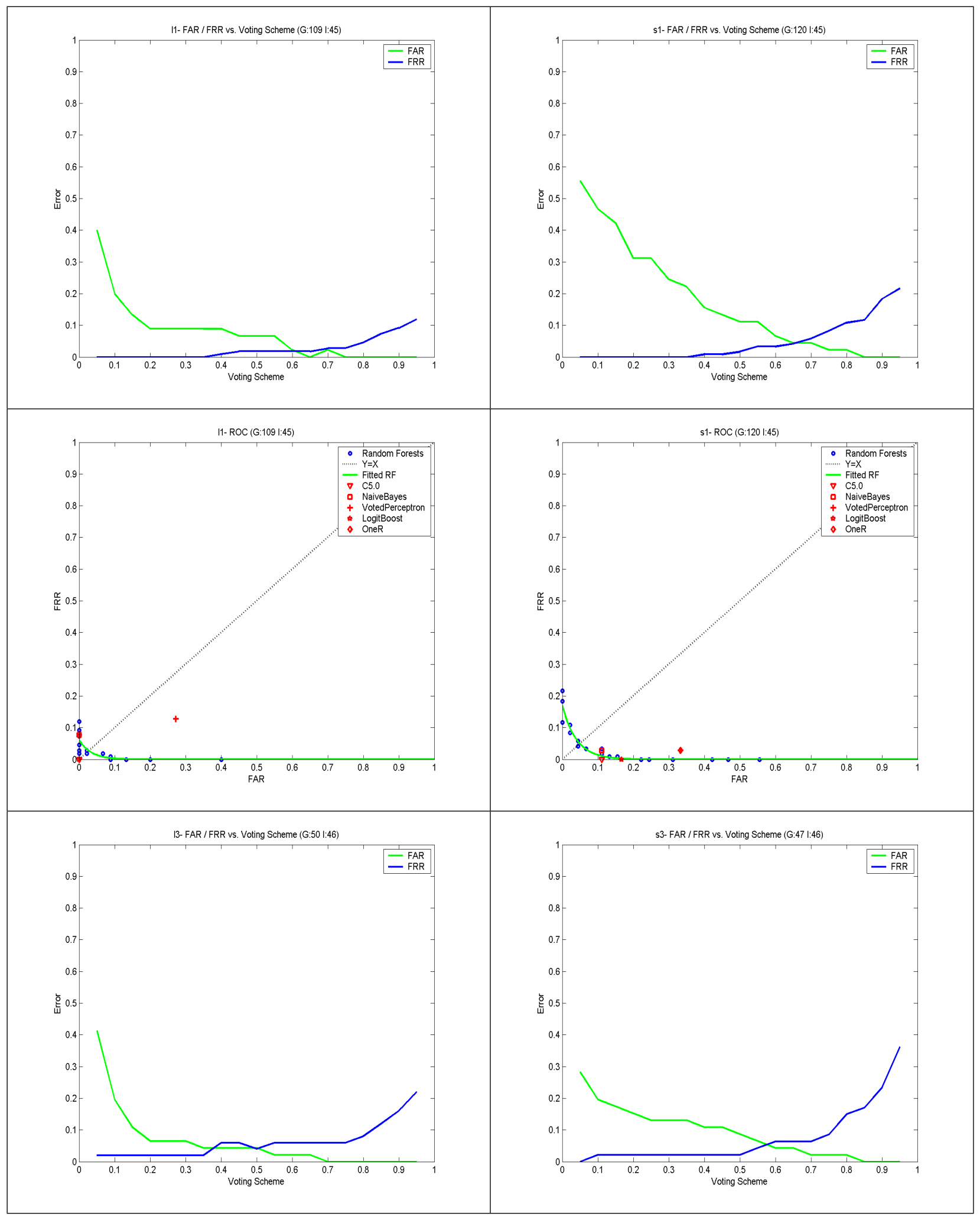




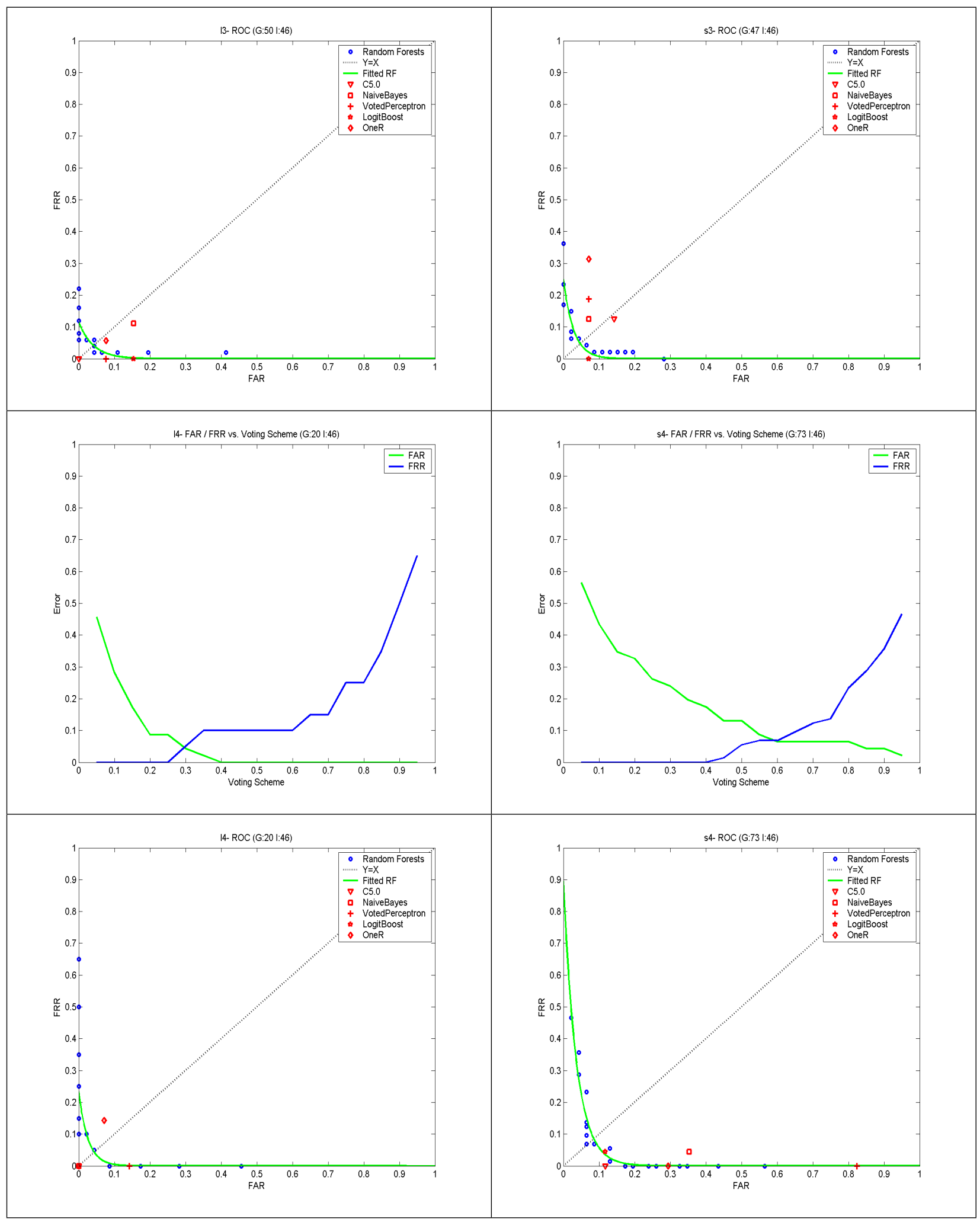




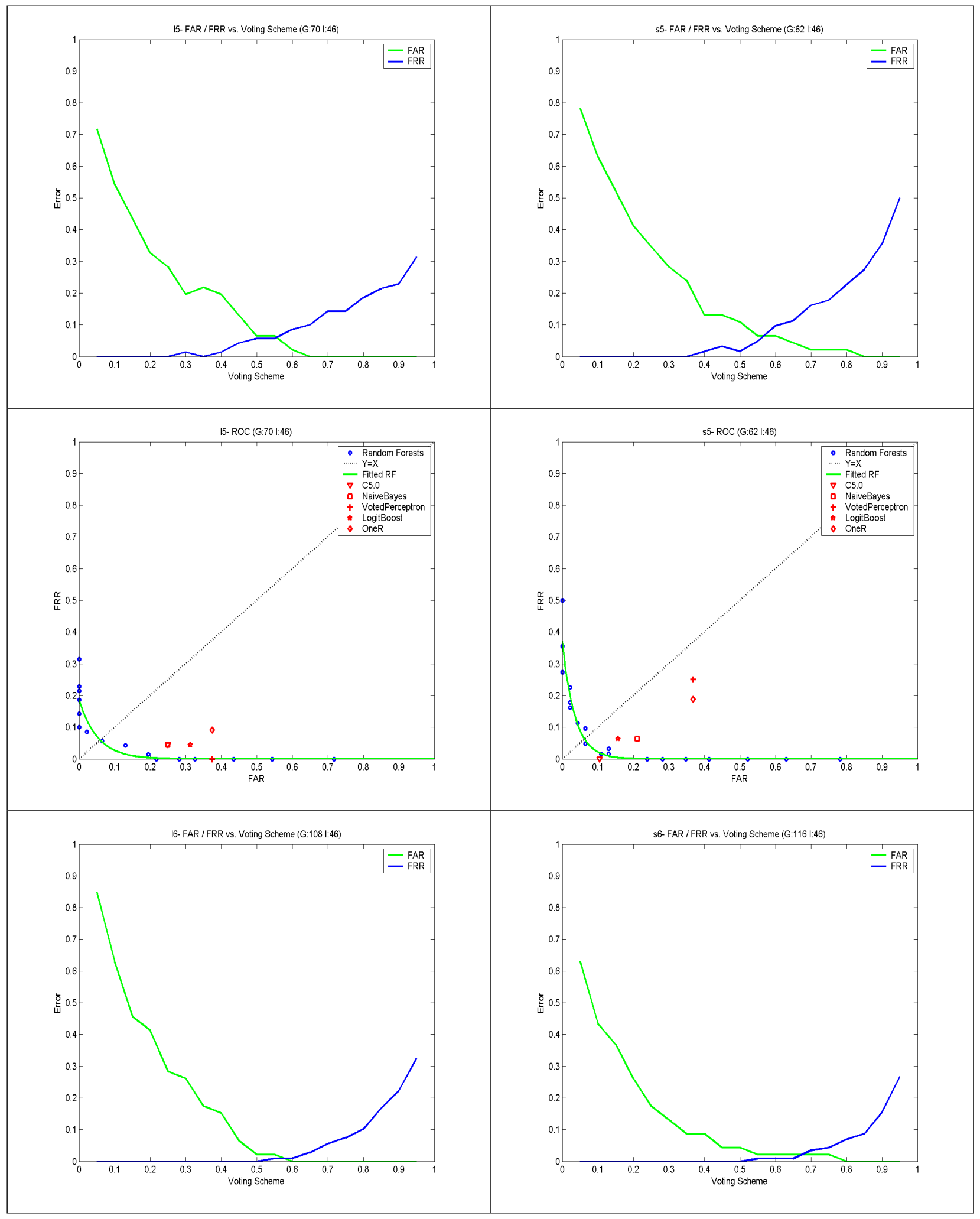




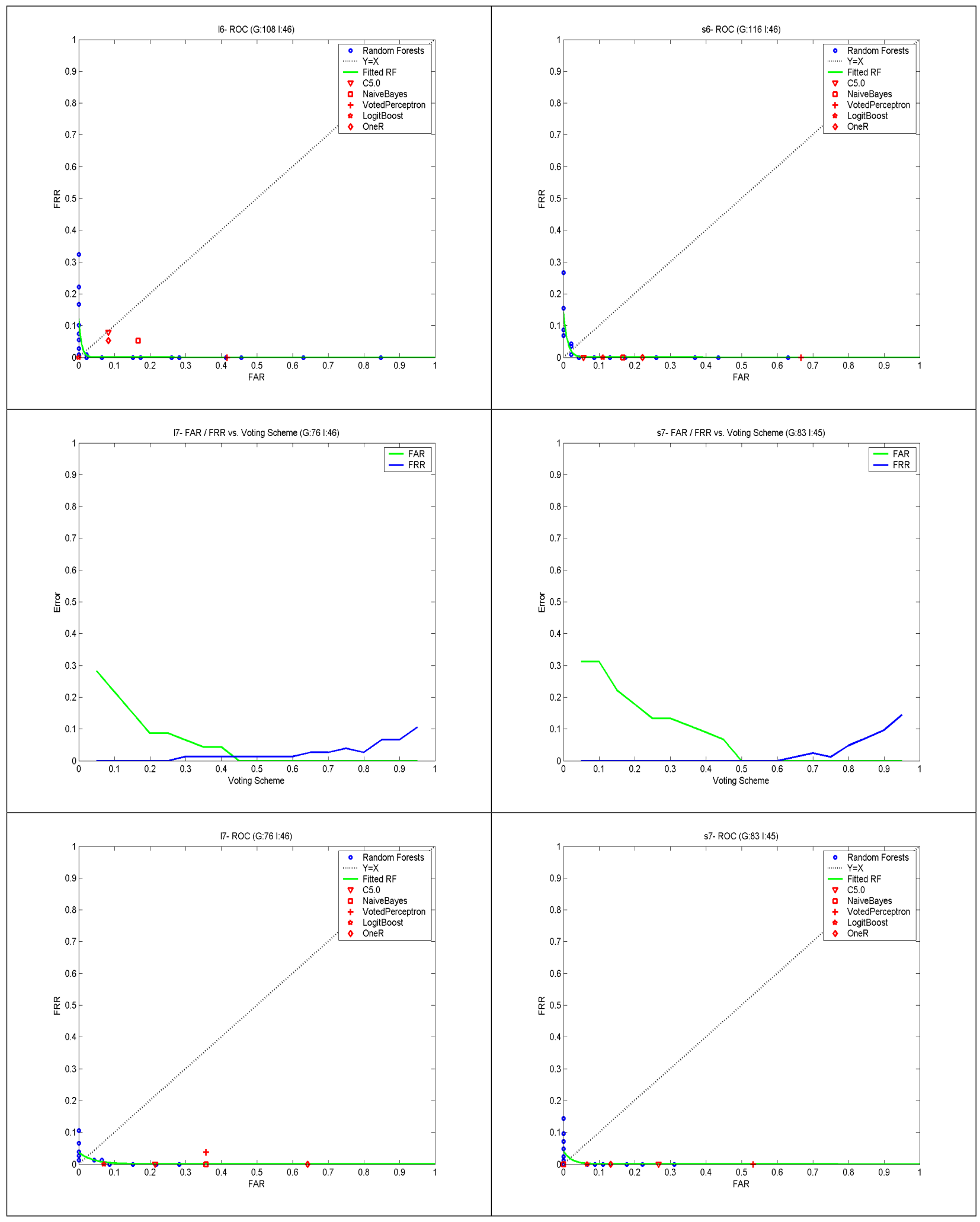




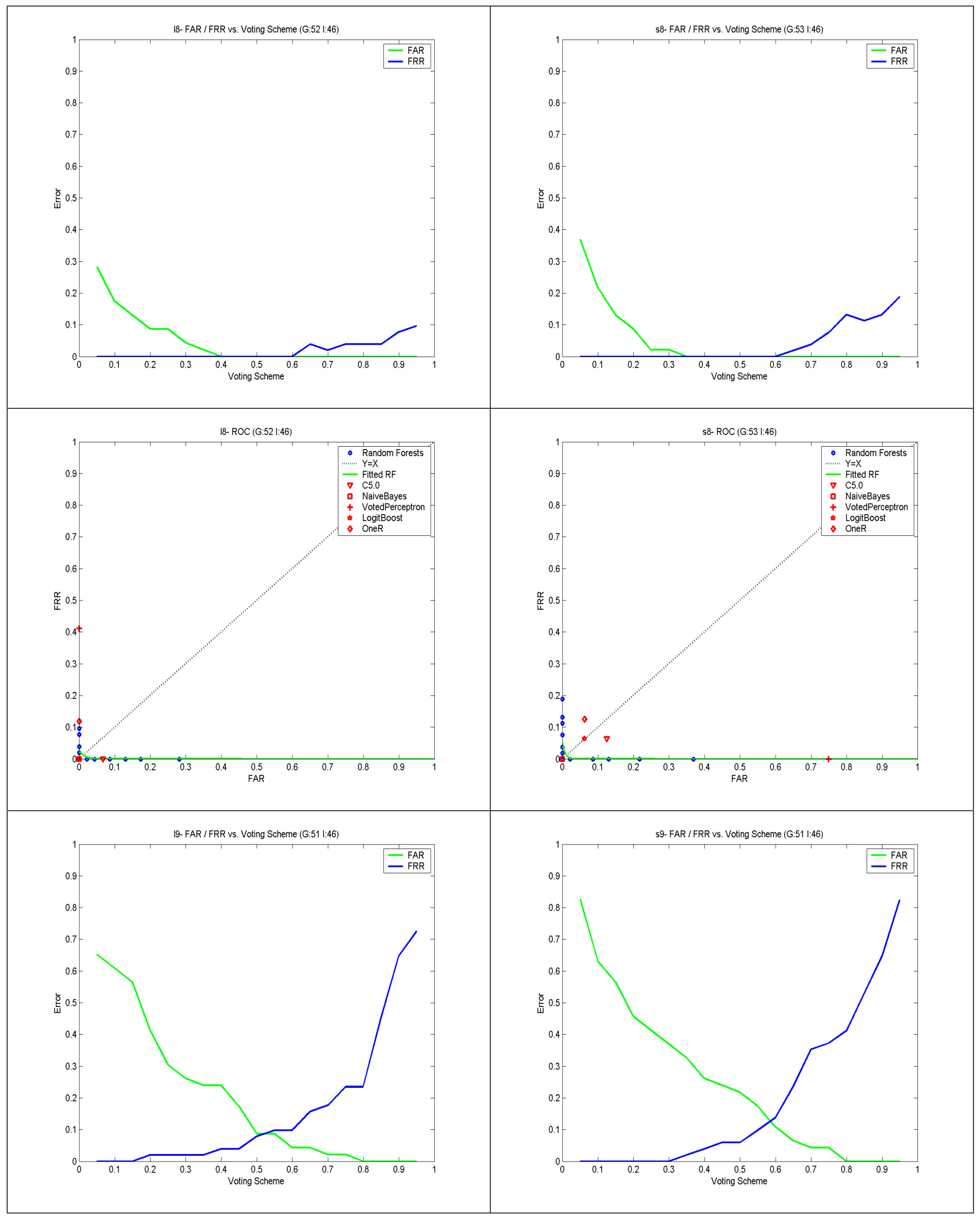




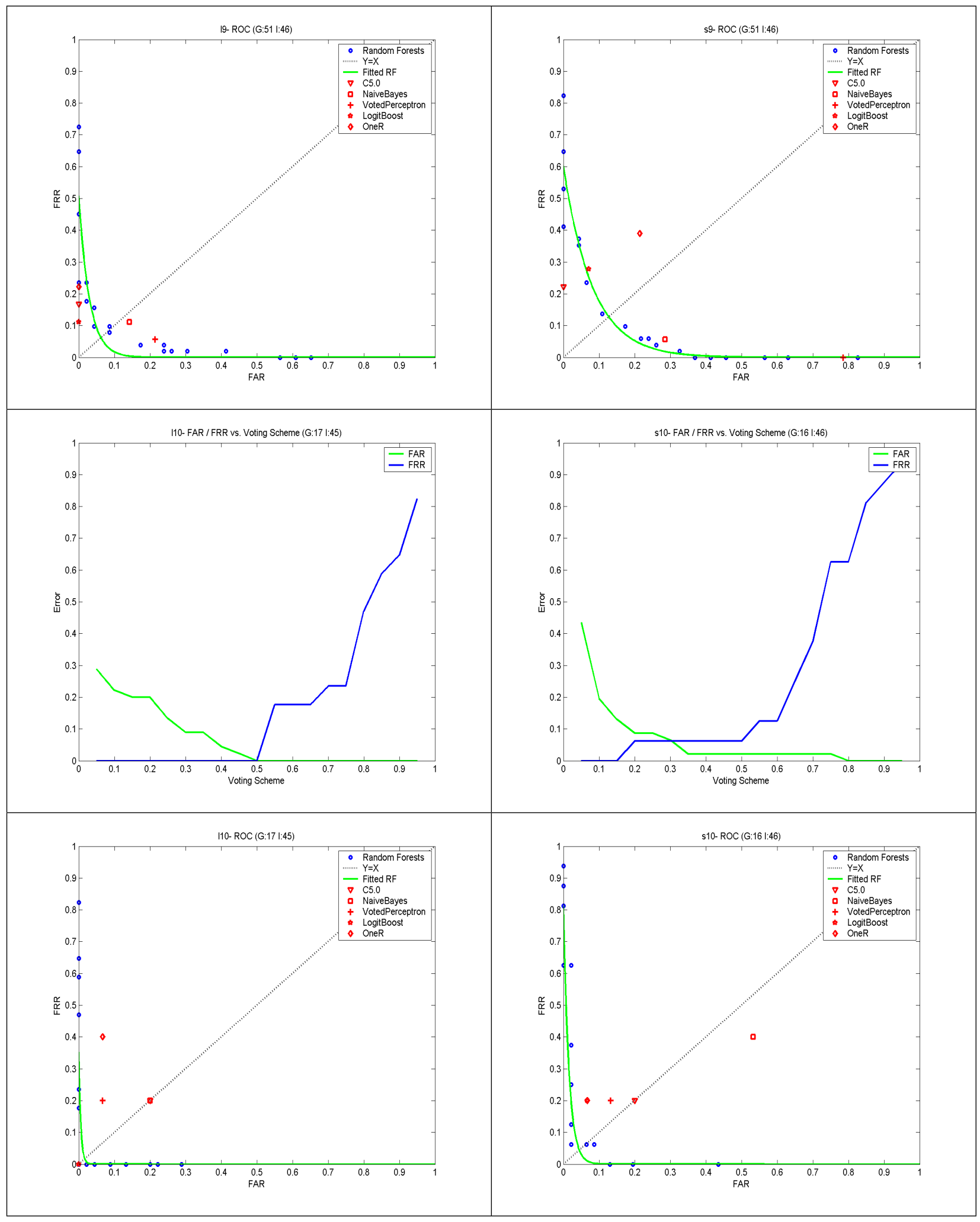




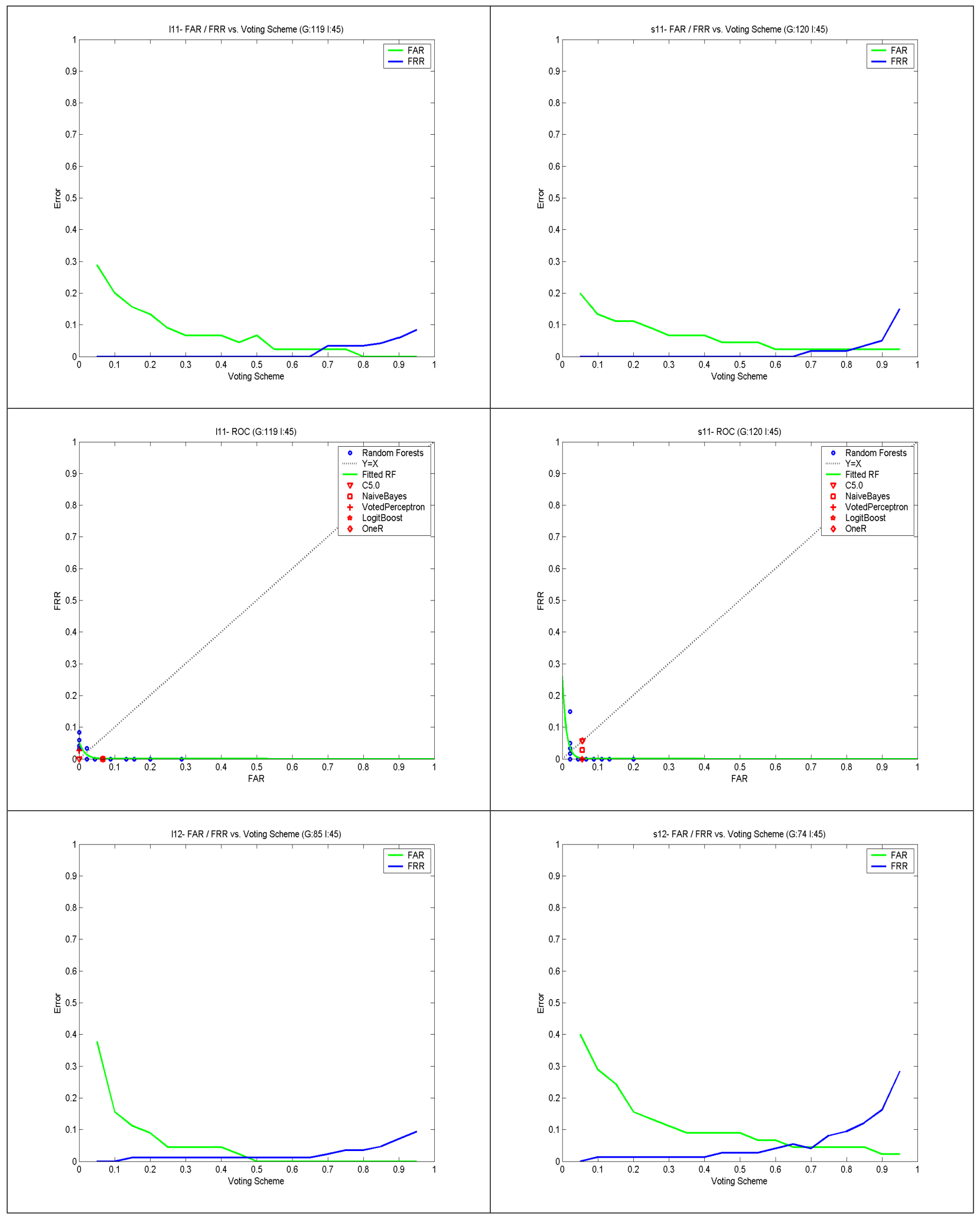




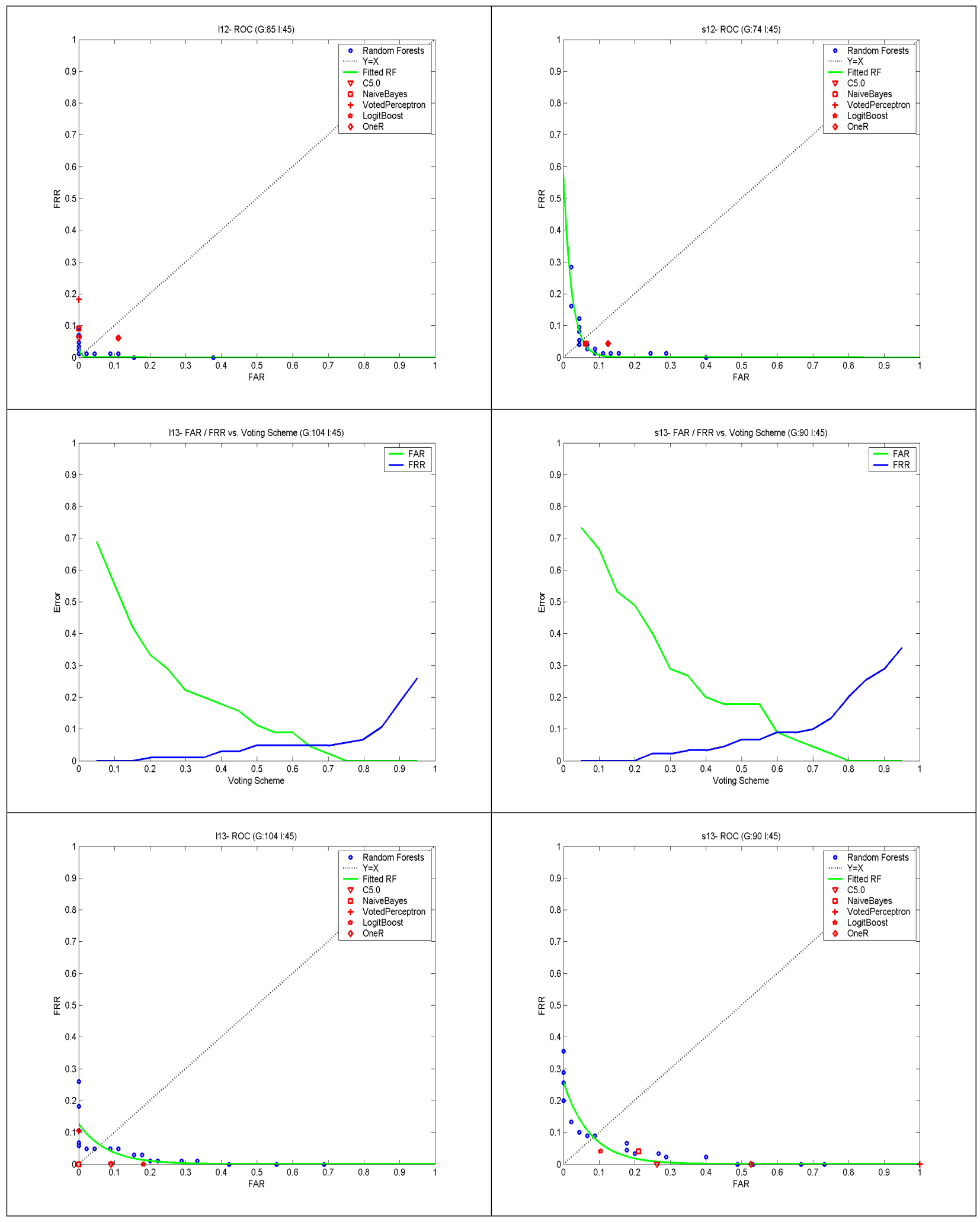




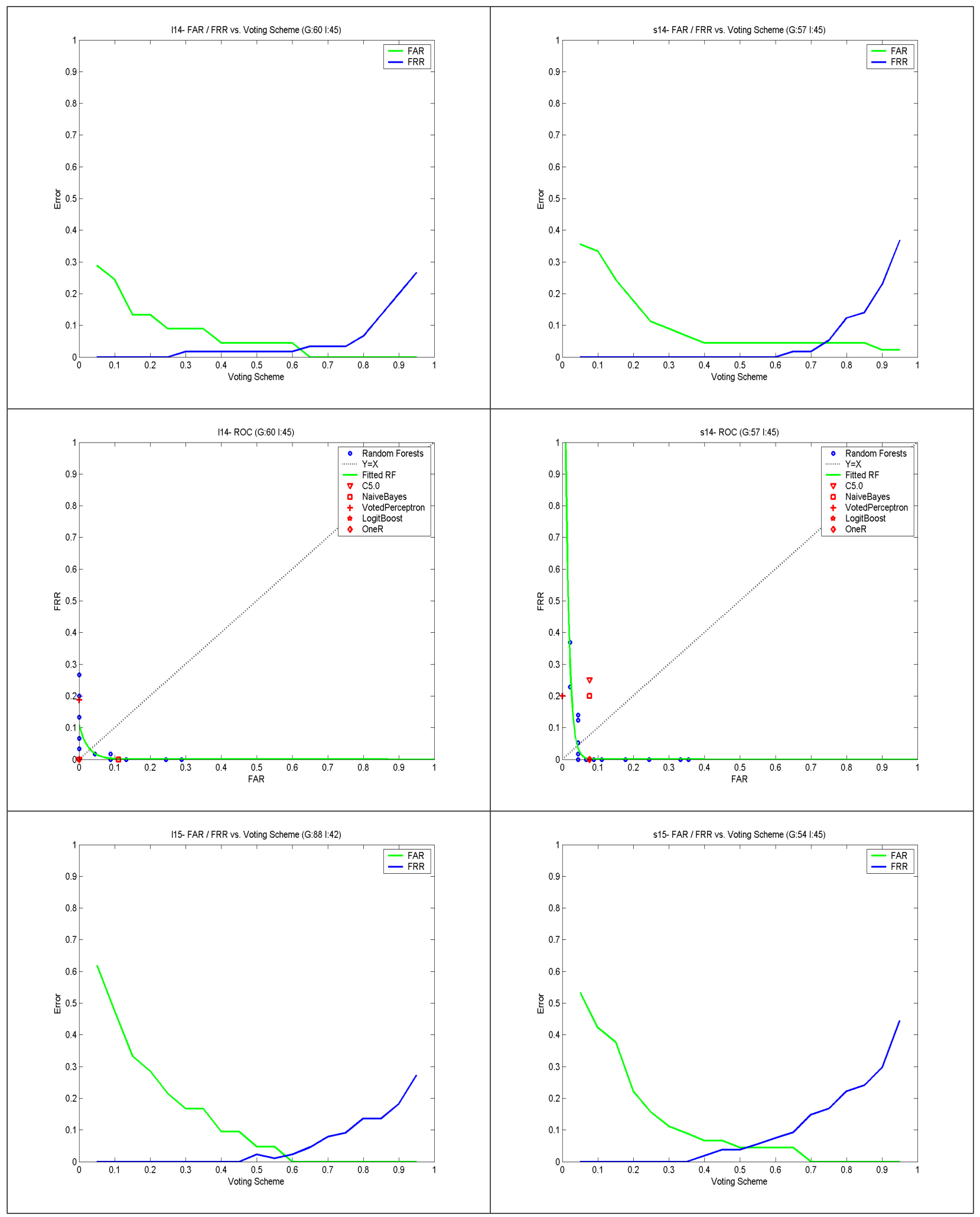




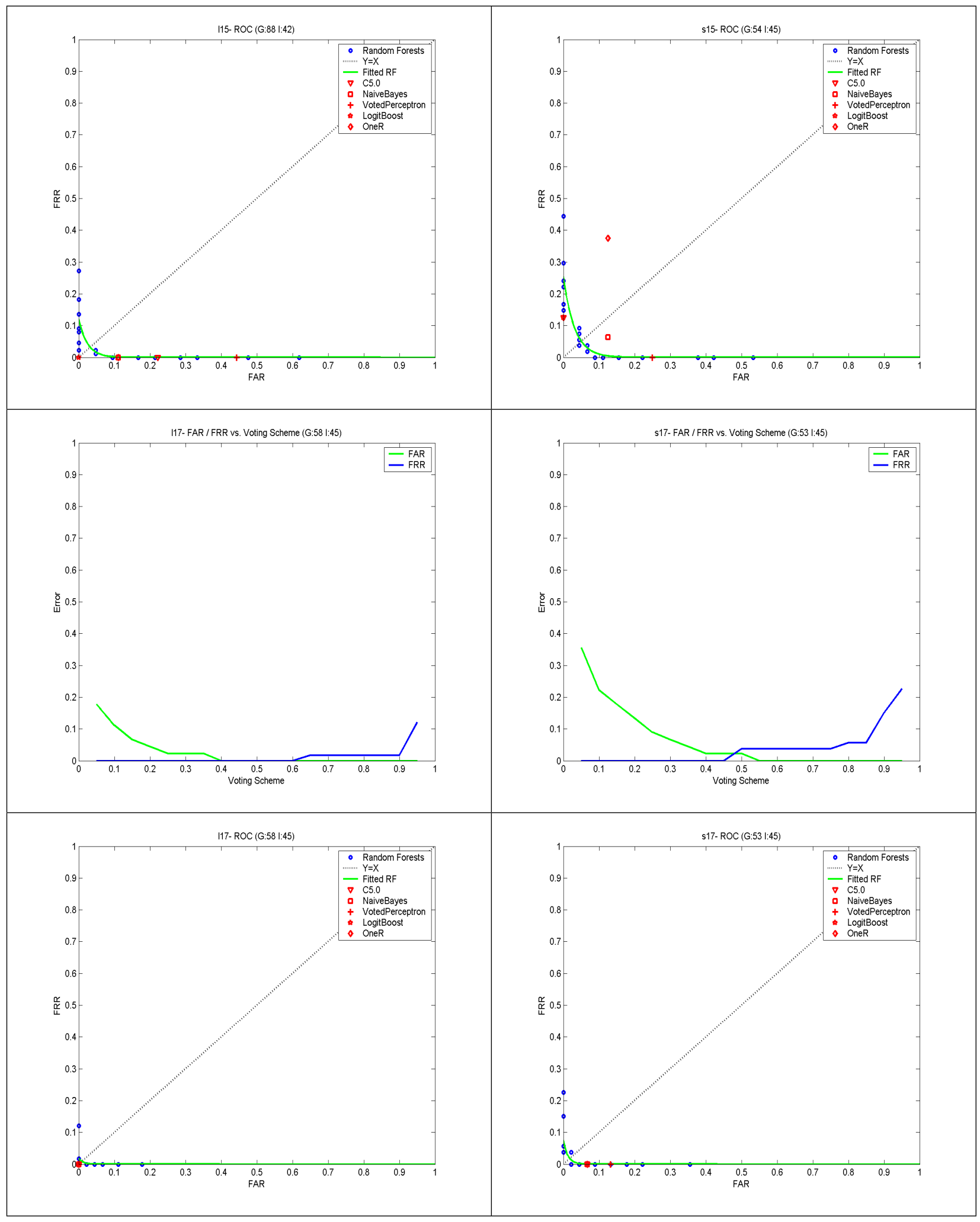




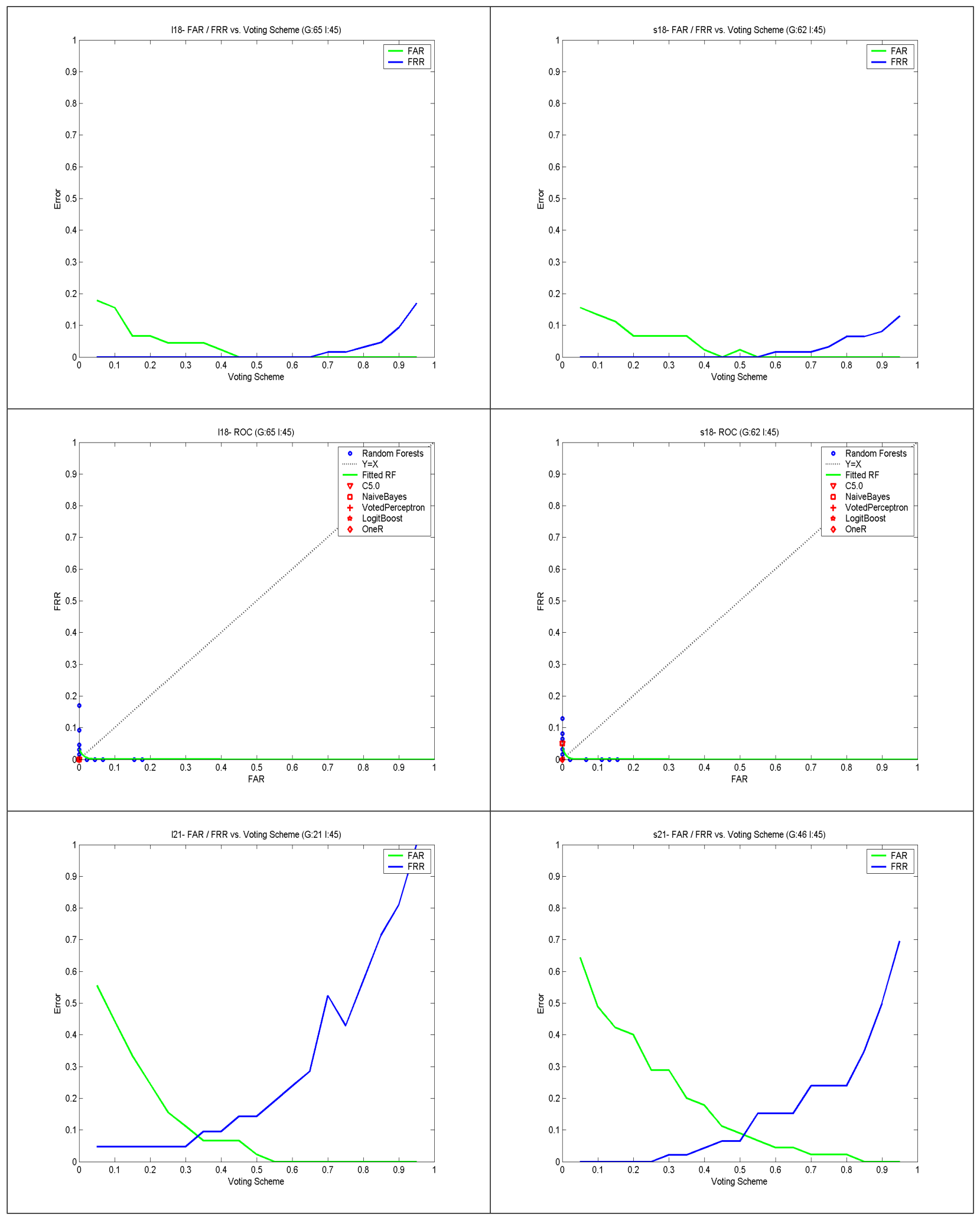




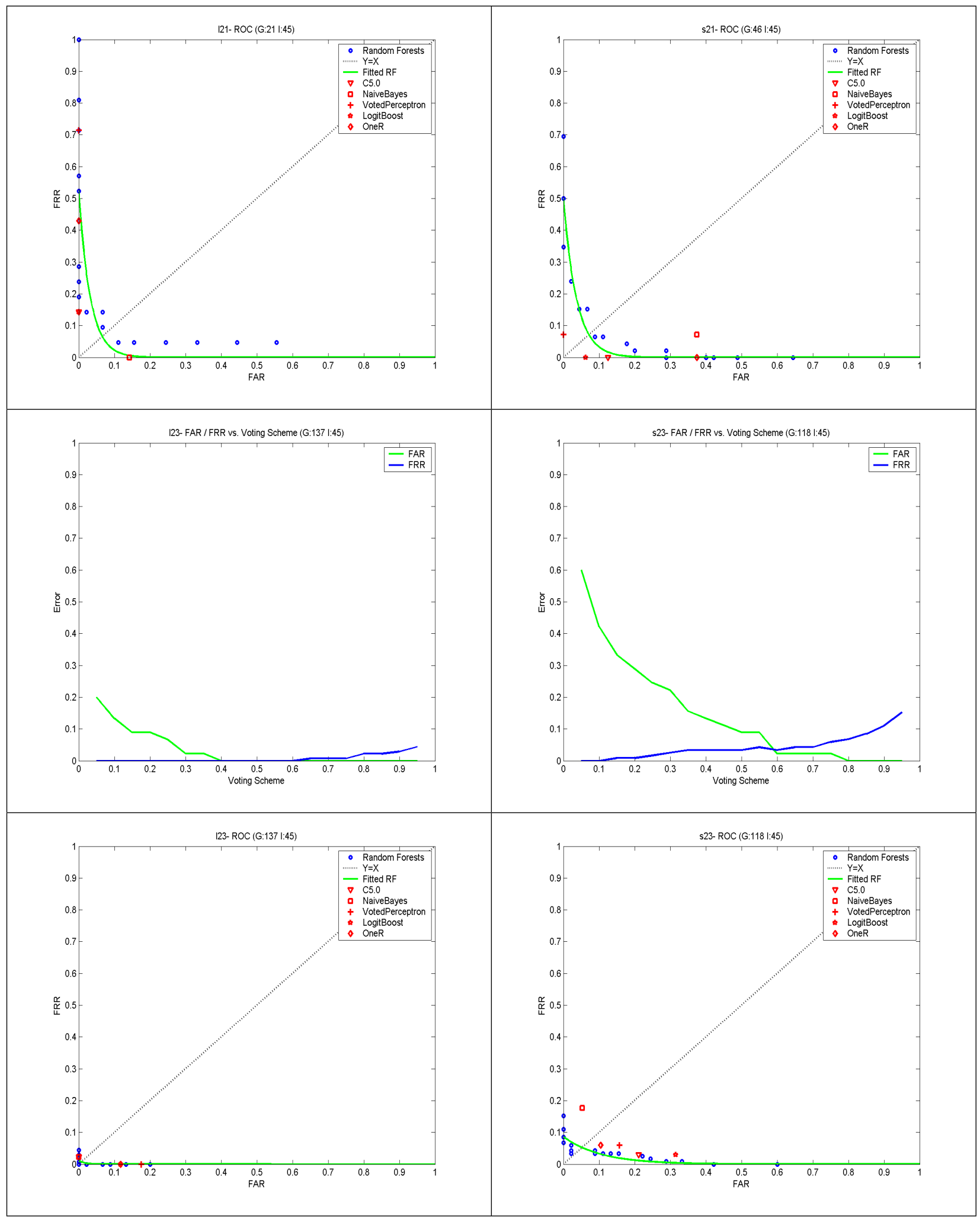




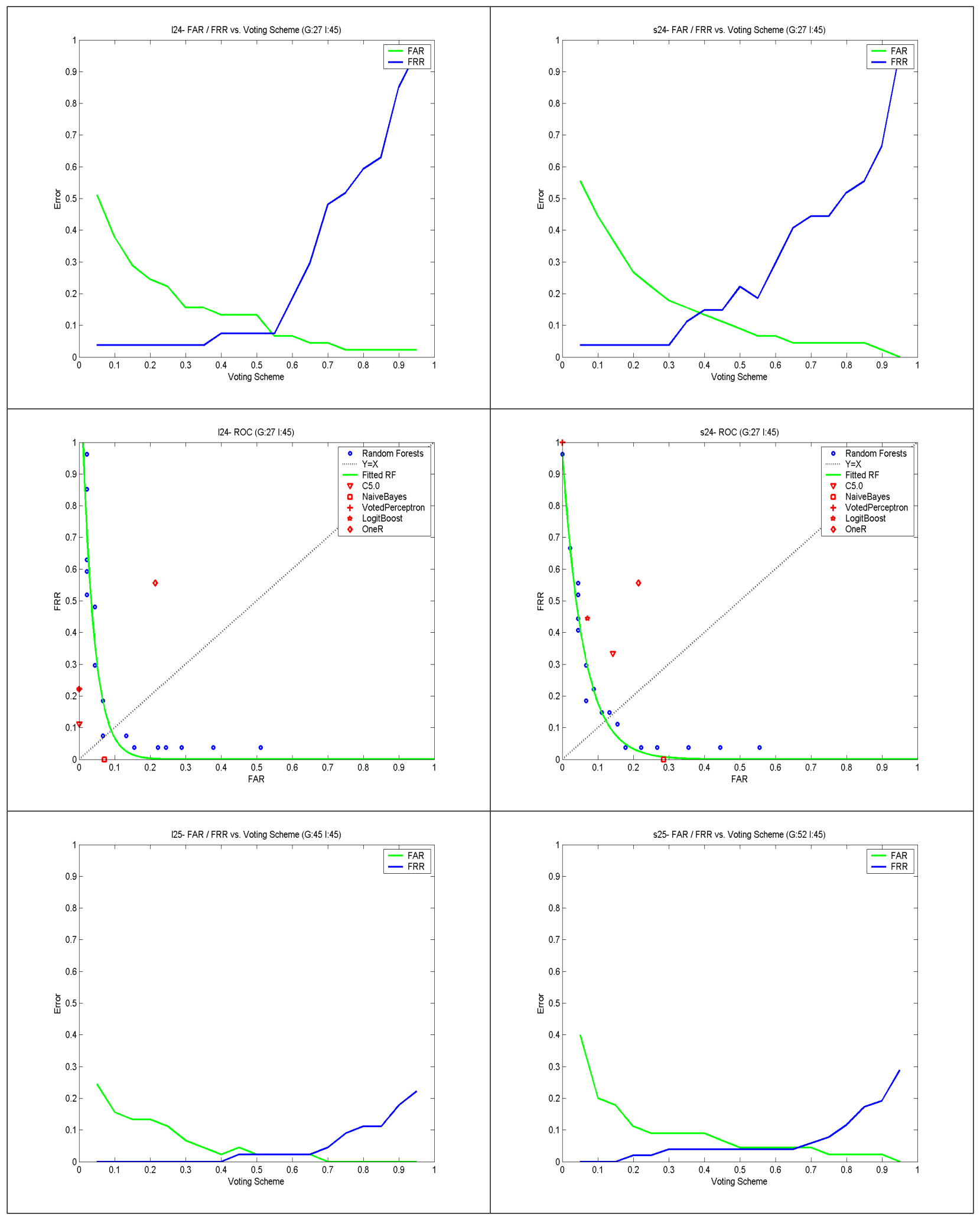




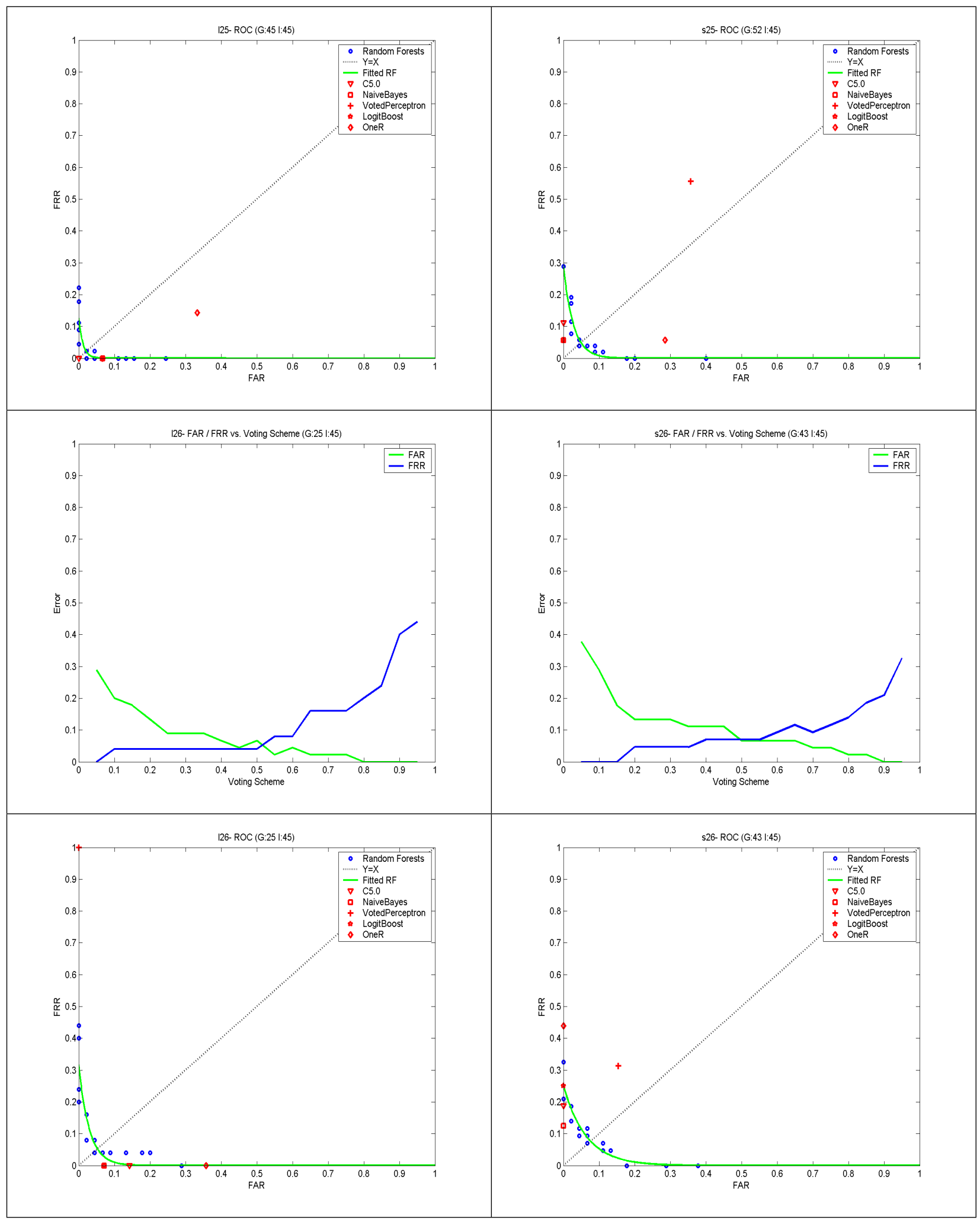




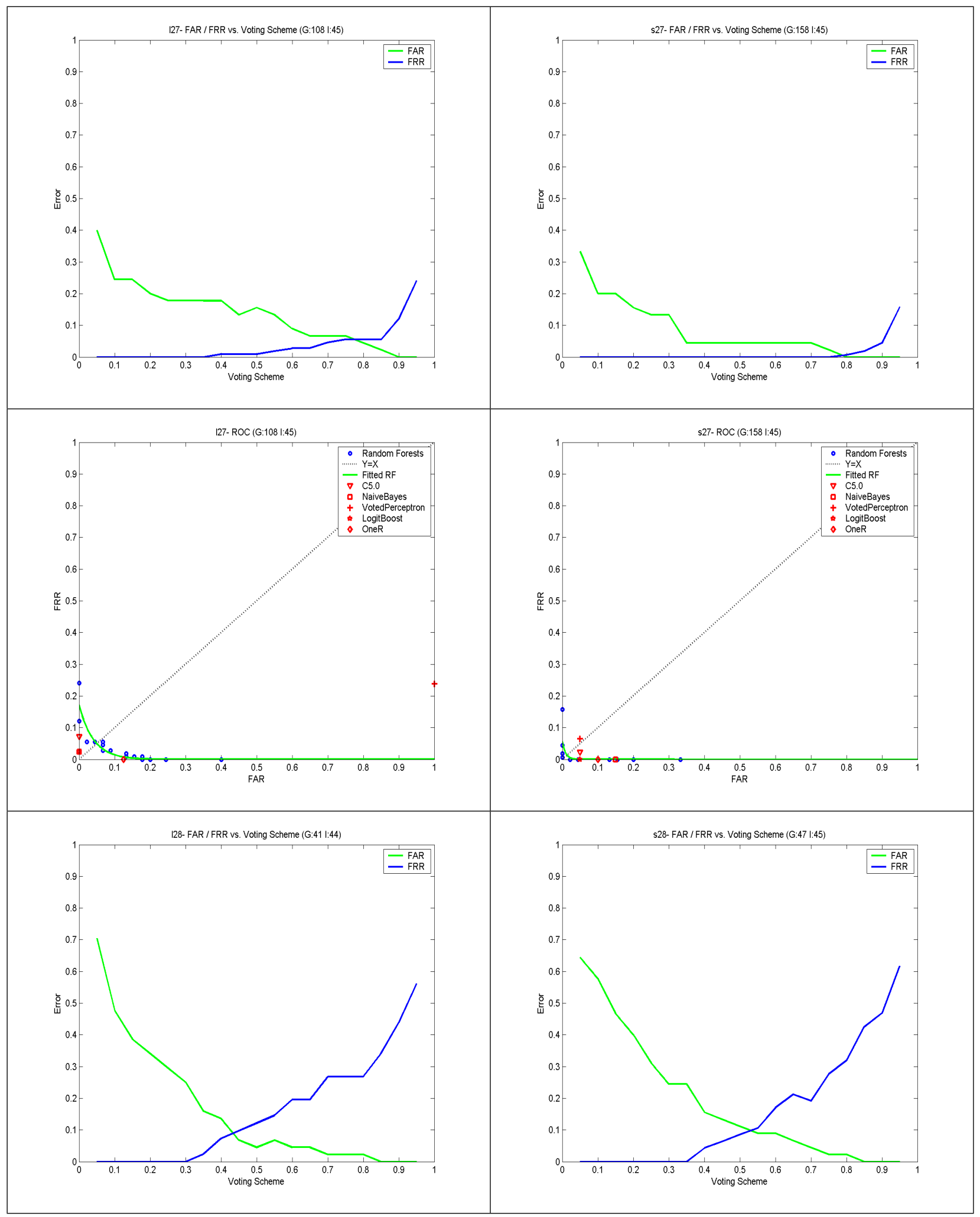




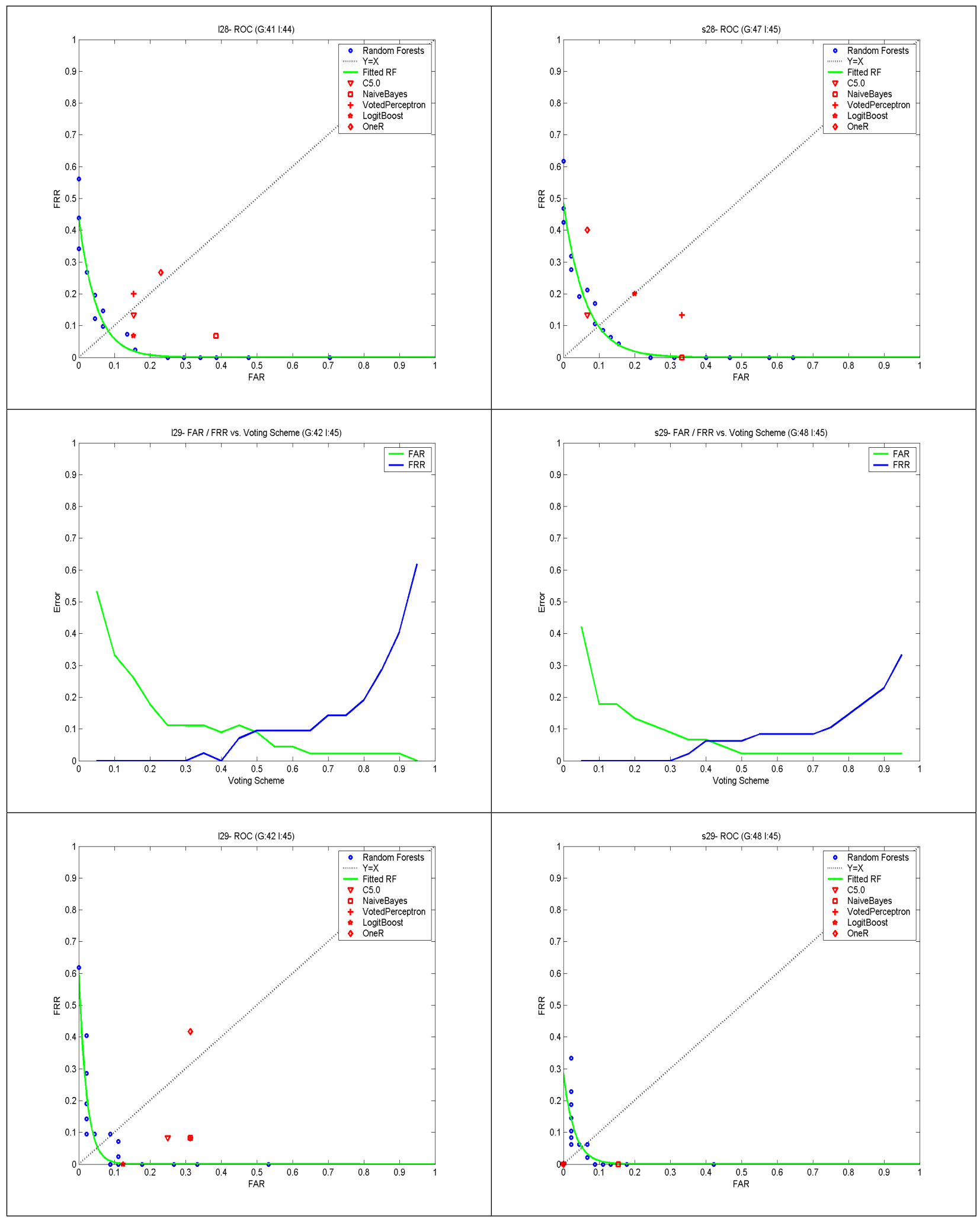




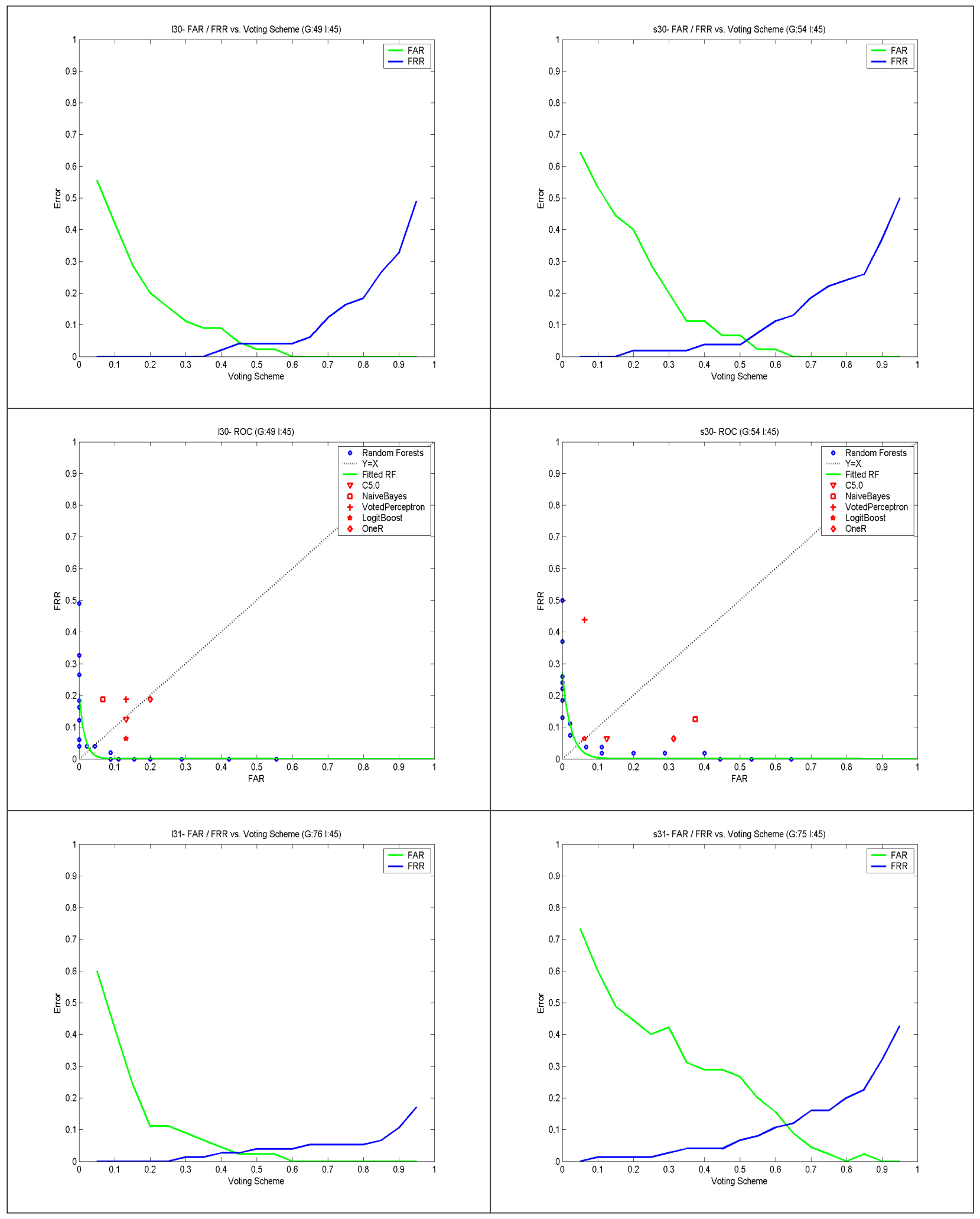




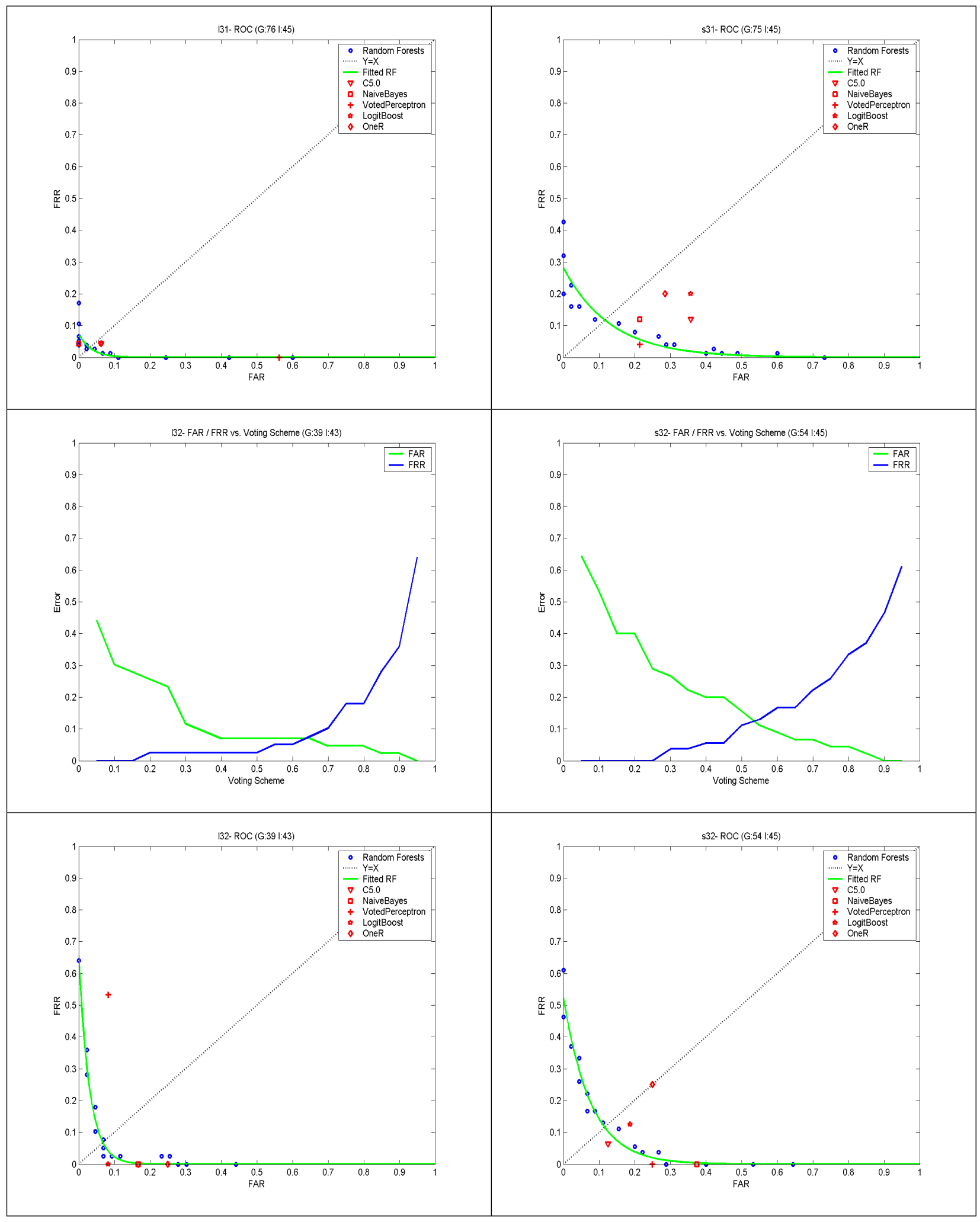




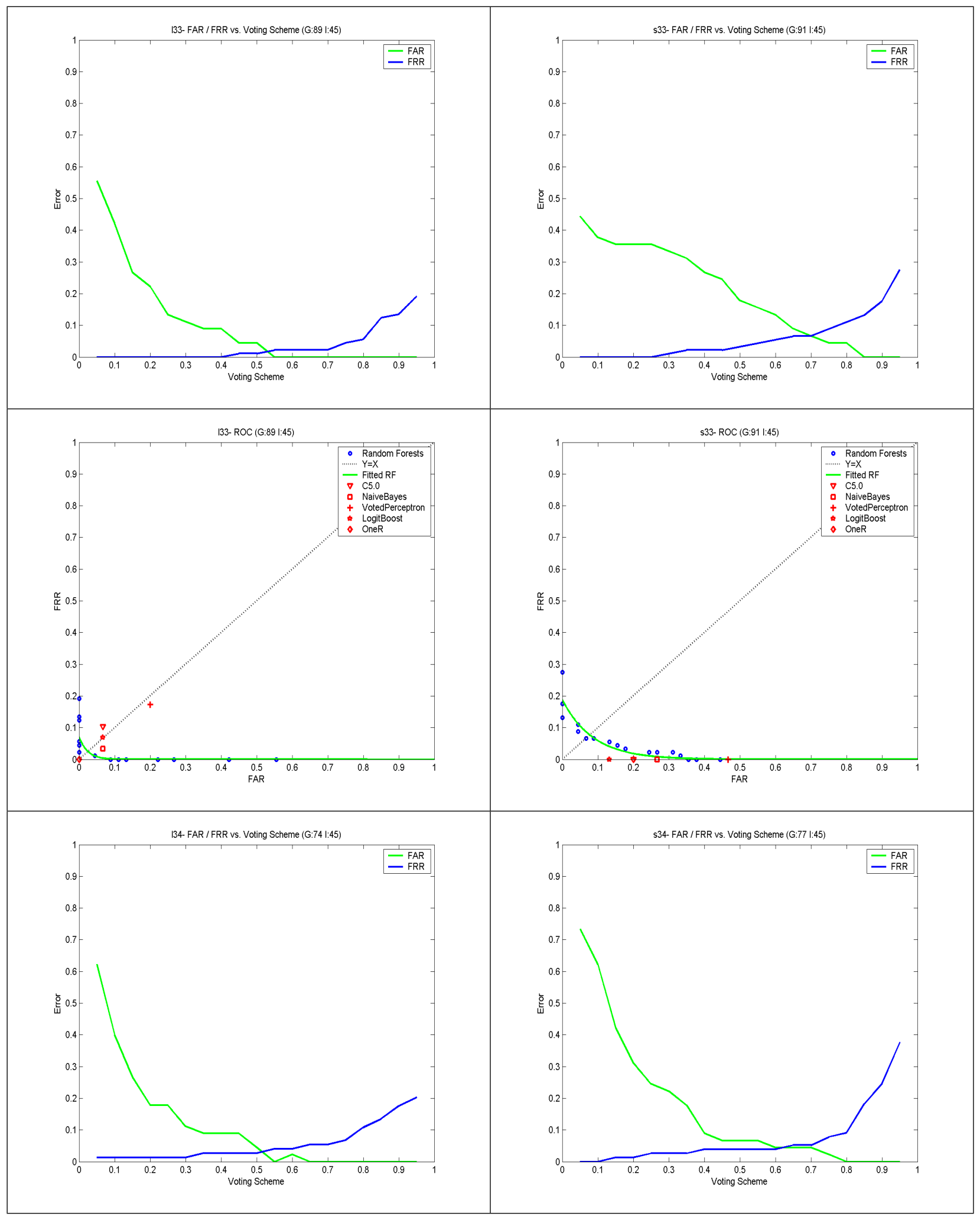




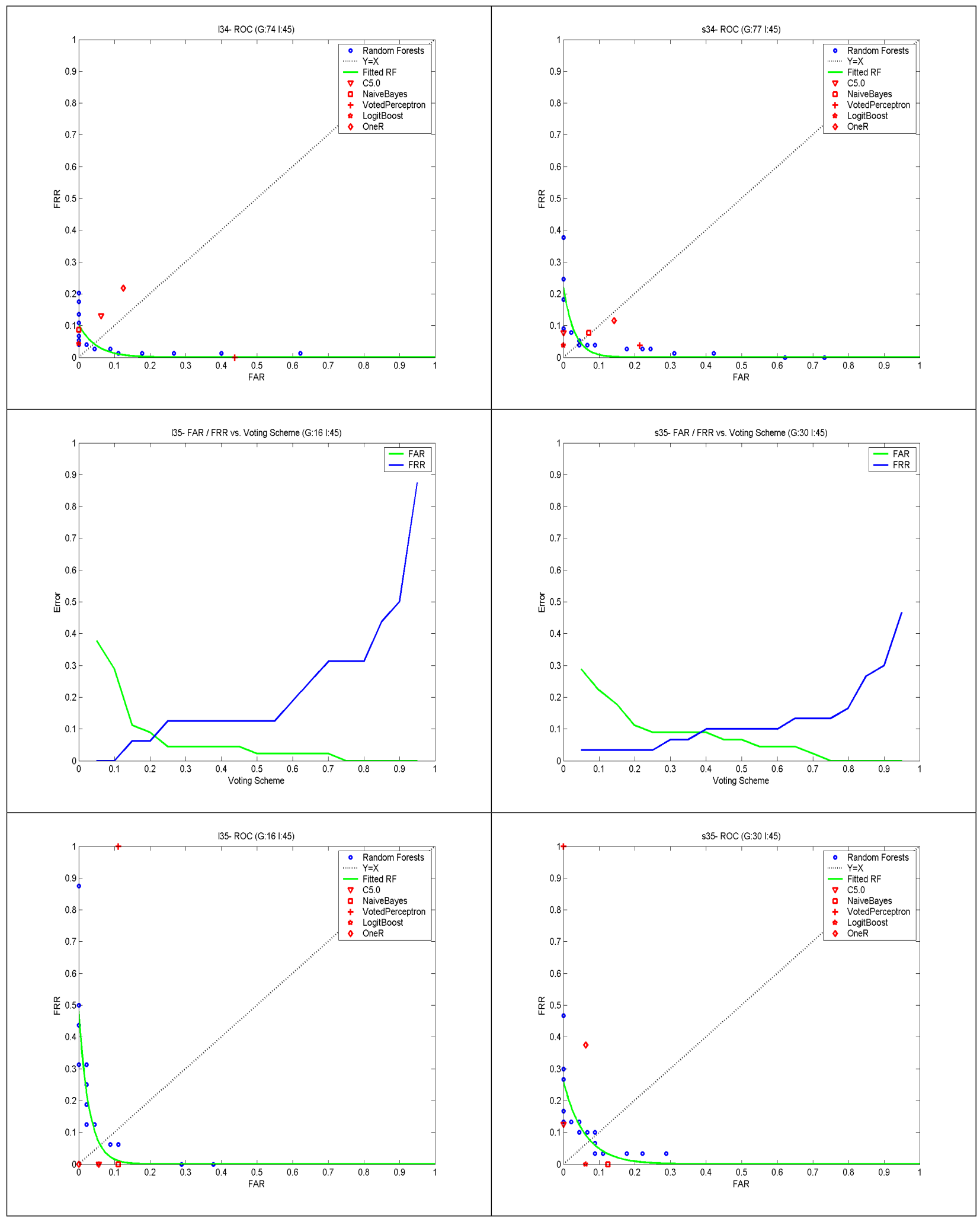




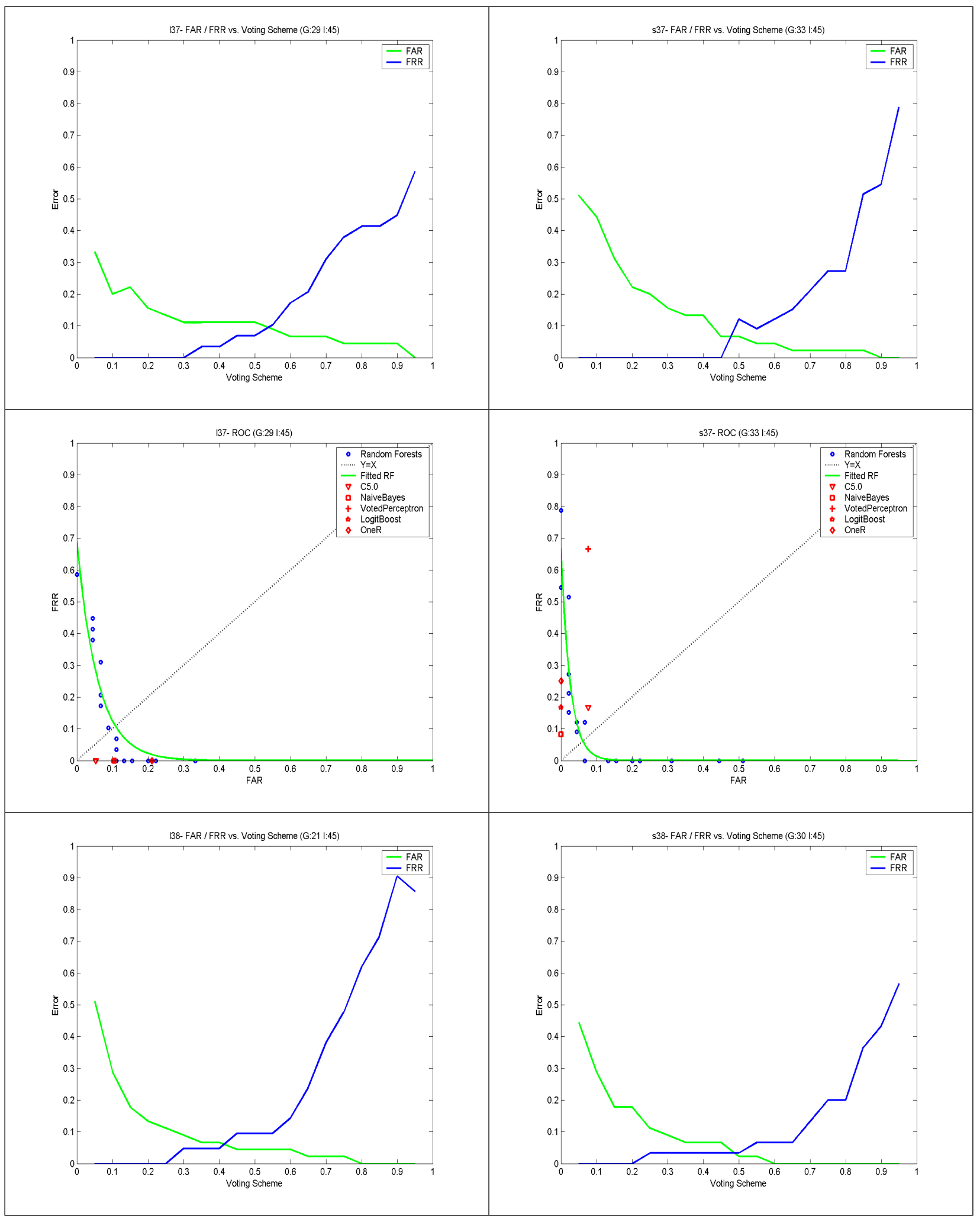




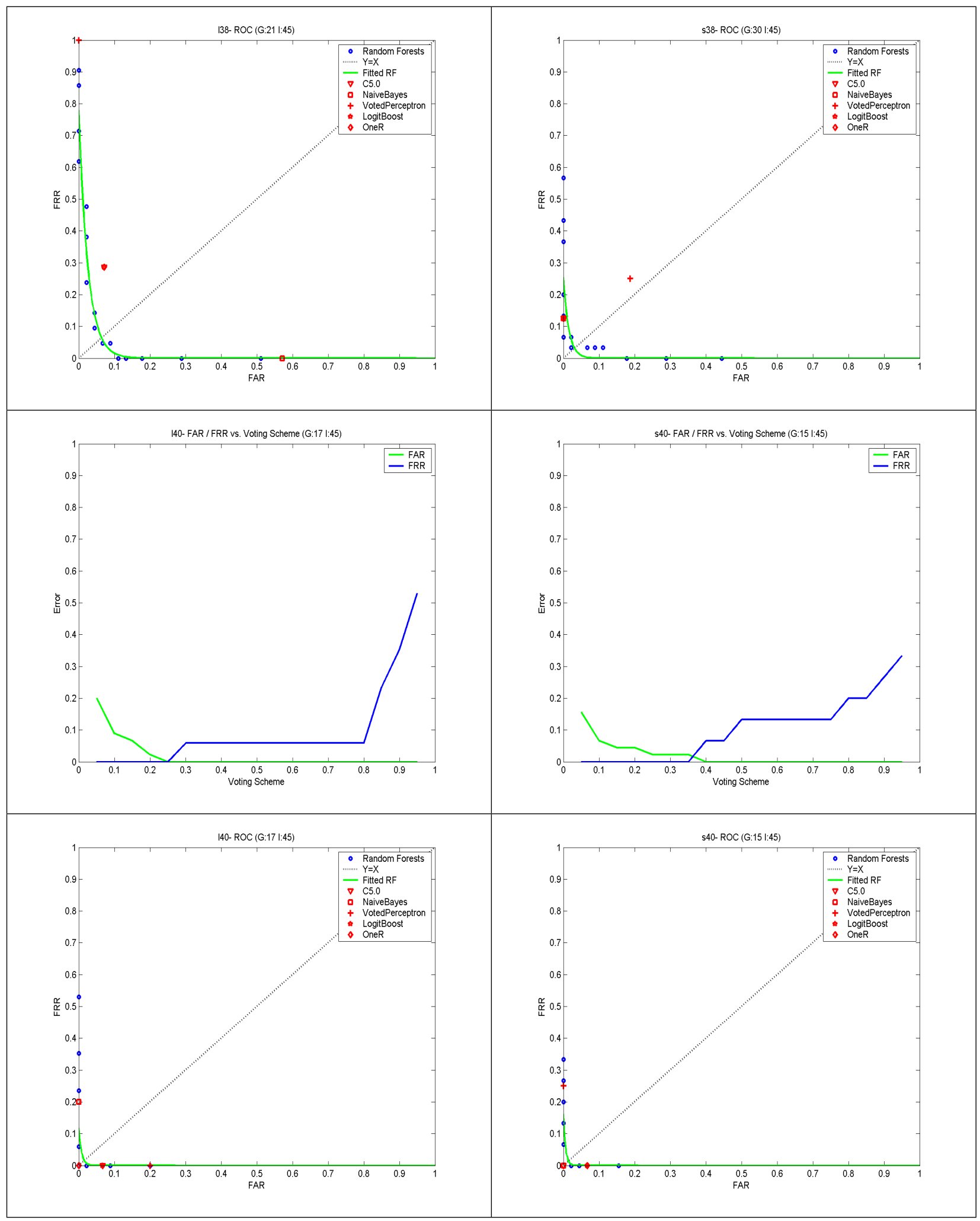




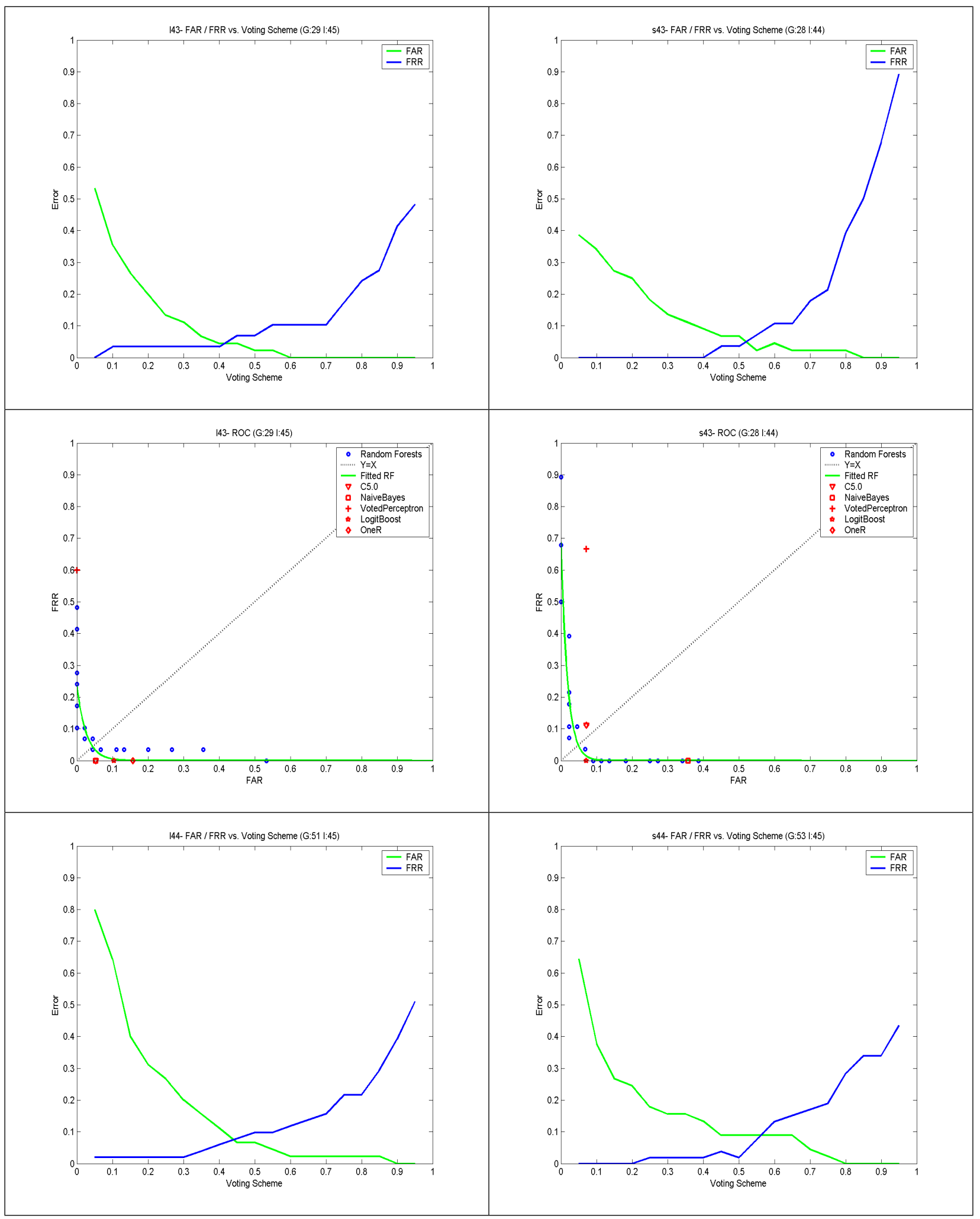




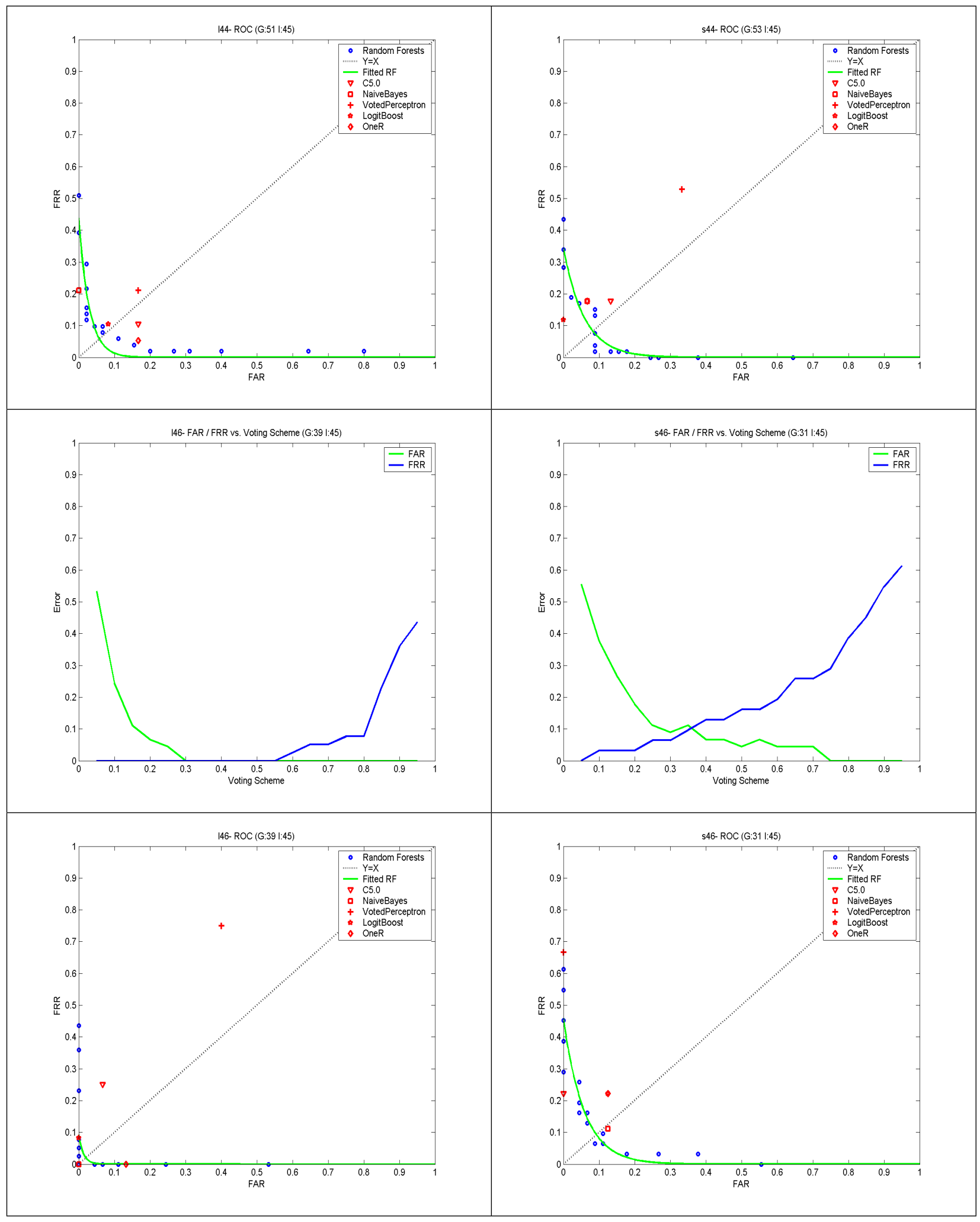




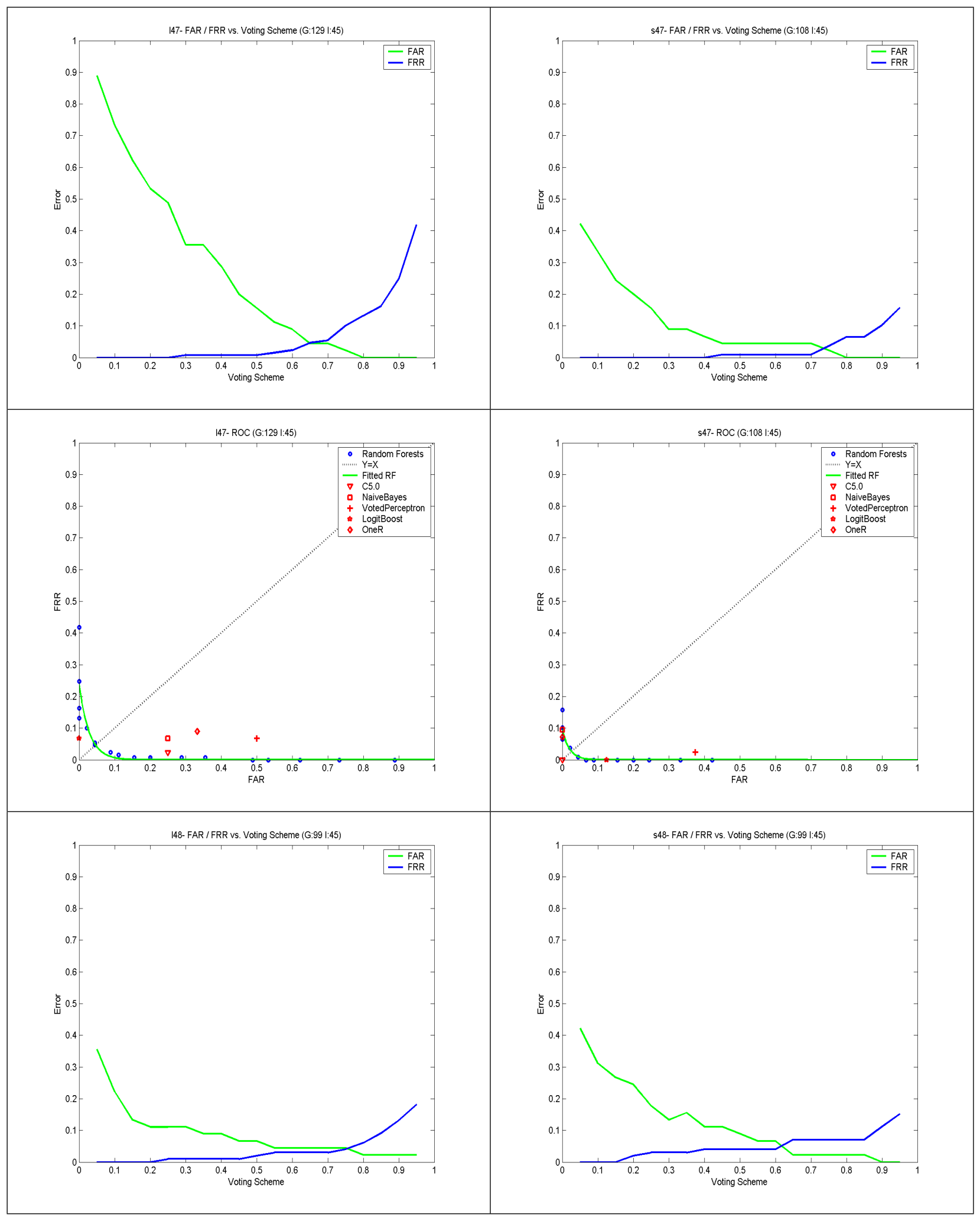




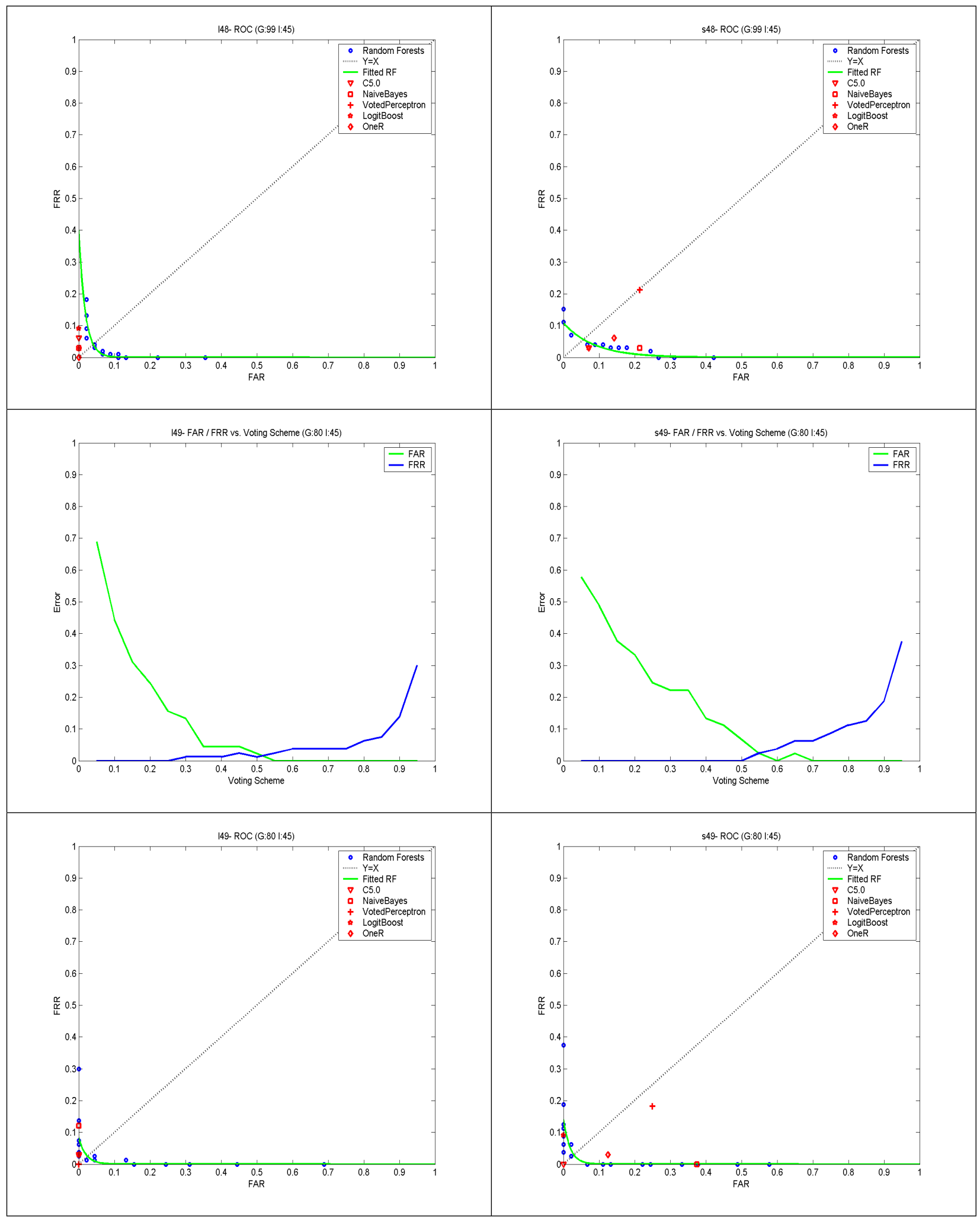




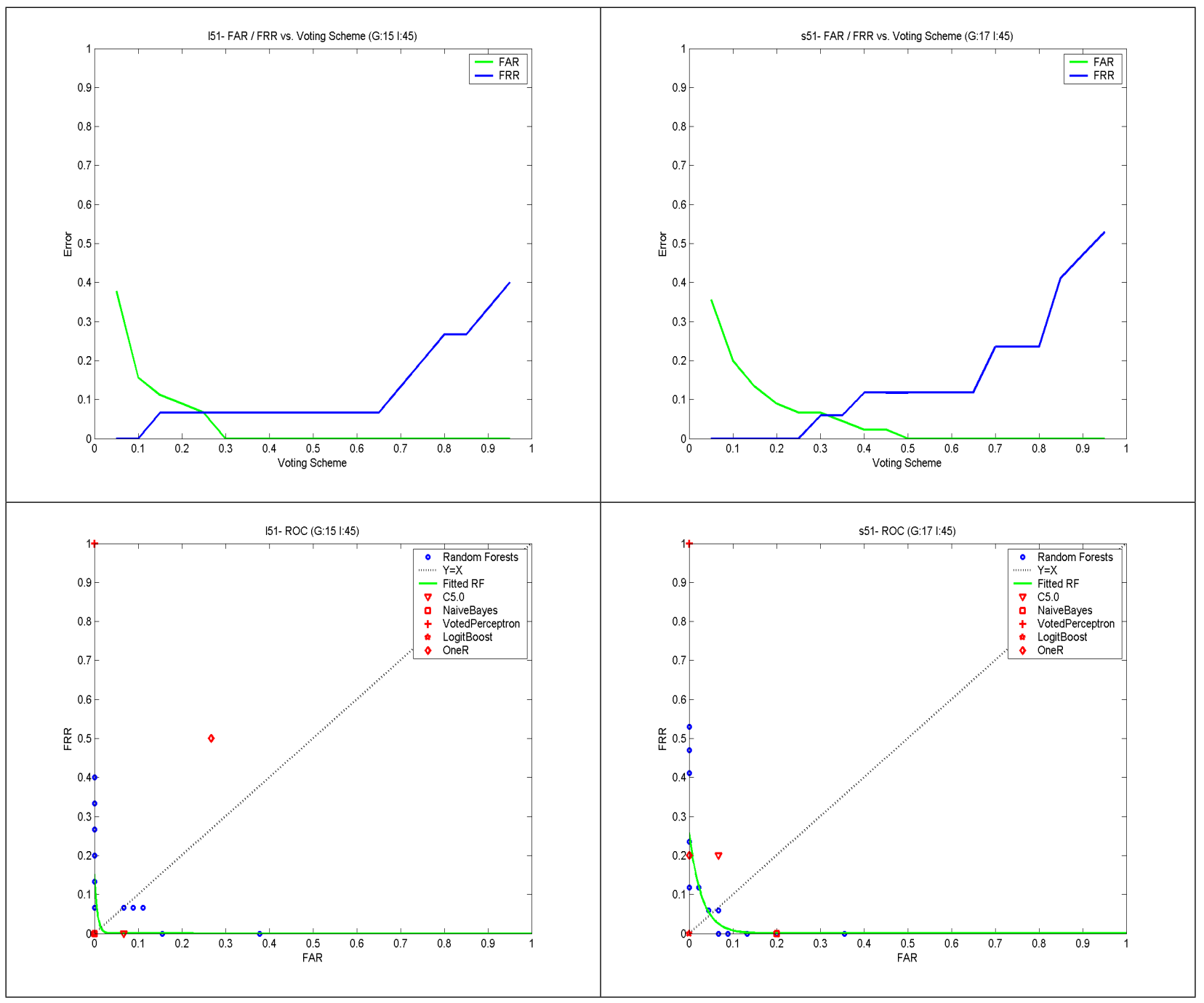




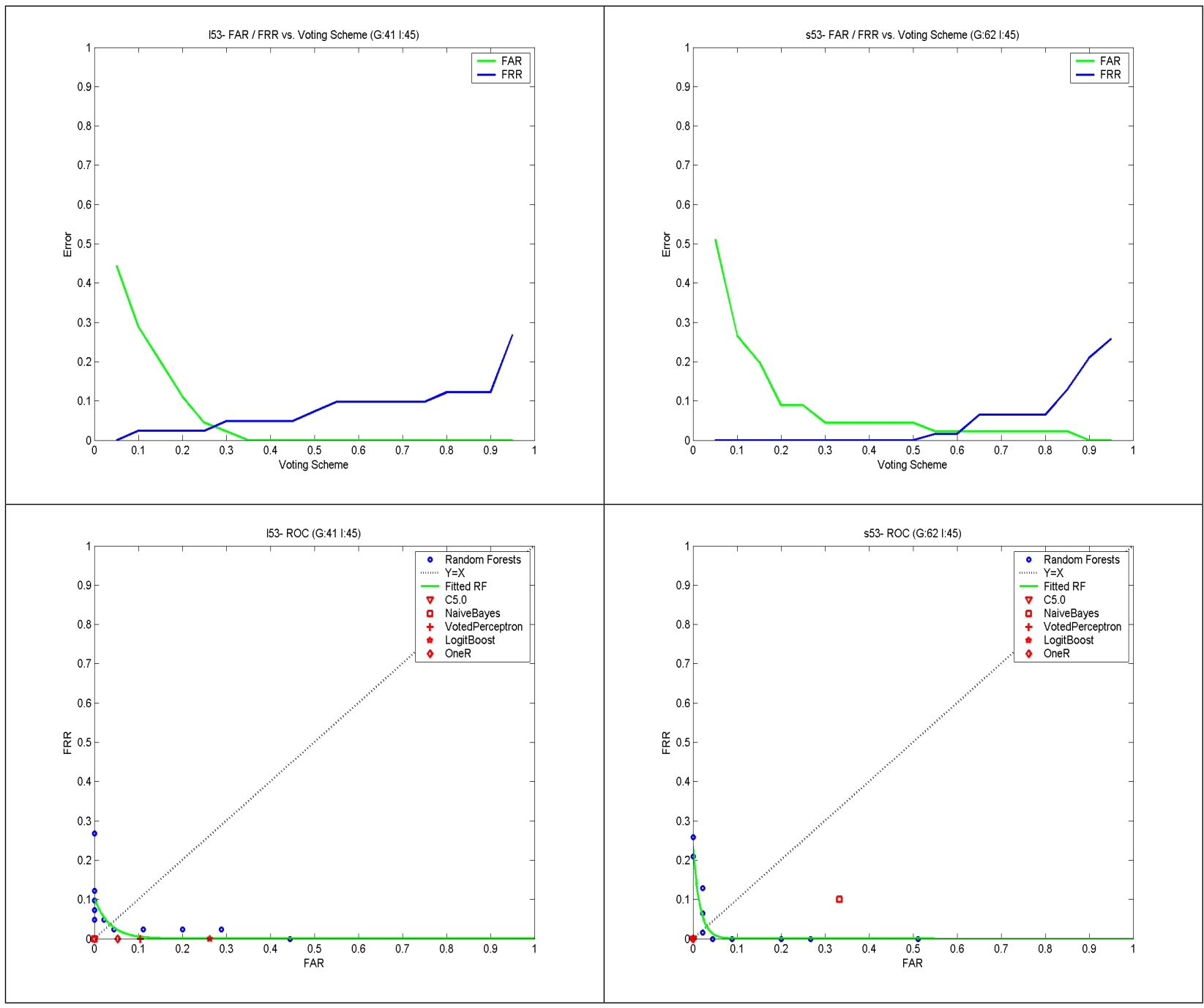





\section{Bibliography}

[1] Charles Beumier, "Facial surface identification," http://www.sic.rma.ac.be/ beumier/3D/3d.html.

[2] Scott Mueller, Upgrading and Repairing PCs, QUE, Indianapolis, IN, 15th edition, 2004.

[3] C. Pfleeger and Shari Pfleeger, Security in computing, Prentice-Hall, Inc., Upper Saddle River, NJ, USA, 2002.

[4] Anil K. Jain, Arun Ross, and Samil Prabhakar, "An introduction to biometric recognition," IEEE Transactions On Circuits And Systems For Video Technology, vol. 14, no. 1, pp. 4-21, 2004, http://www.csee.wvu.edu/ ross/courses/ sp05/biom693/reading/RossBioIntro_CSVT2004.pdf.

[5] National Center for State Courts, "Fingerprint," http://ctl.ncsc.dni.us/biomet\%20web/BMFingerprint.html.

[6] Arun Ross, "Class Notes: BIOM 693 West Virginia University Spr '05,” Jan 2005.

[7] N K Ratha, J H Connell, and R M Bolle, "Enhancing security and privacy in biometrics-based authentication systems," IBM Systems Journal, vol. 40, no. 3, 2001.

[8] Ian H. Witten and Eibe Frank, Data Mining: Practical Machine Learning Tools and Techniques with Java Implementations, Morgan-Kaufman, San Francisco.

[9] Wikipedia, "Machine learning," http://en.wikipedia.org/wiki/Machine_learning.

[10] Dictionary.com, "Data mining," 2003, http://dictionary.reference.com/search?q=data+mining.

[11] Wikipedia, "Data mining," http://en.wikipedia.org/wiki/Data_mining.

[12] Dictionary.com, "Pattern recognition," Sep 1995, http://dictionary.reference.com/search?q=pattern+recognition.

[13] S. Singh, The Code Book: The Science of Secrecy from Ancient Egypt to Quantum Cryptography, Anchor Books, NY, 1999. 
[14] R Gaines, W Lisowksi, W Press, and S Shapiro, "Authentication by keystroke timing: Some preliminary results," Rand Report R-256-NSF, The Rand Corporation, Santa Monica, CA, 1980.

[15] J Garcia, "Personal identification apparatus," Patent 4,621,334, U.S. Patent and Trademark Office, Washington, D.C., 1986.

[16] J R Young and R W Hammon, "Method and apparatus for verifying an individuals identity," Patent 4,805,222, U.S. Patent and Trademark Office, Washington, D.C., 1989.

[17] R Joyce and G Gupta, "Identity authentication based on keystroke latencies," Communications of the ACM, vol. 33, no. 2, Feb 1990.

[18] M S Obaidat and D T Macchairolo, "An on-line neural network system for computer access security," IEEE Trans. Industrial Electronics, vol. 40, no. 2, pp. 235-241, Apr 1993.

[19] S Bleha and M S Obaidat, "Computer user verification using the perceptron," IEEE Trans. Systems, Man, and Cybernetics, vol. 23, no. 3, pp. 900-902, May 1993.

[20] M S Obaidat and B Sadoun, "Verification of computer users using keystroke dynamics," IEEE Trans. Systems, Man, and Cybernetics, vol. 27, no. 2, pp. 261-269, Apr 1997.

[21] Fabian Monrose, Michael K. Reiter, and Susanne Wetzel, "Password hardening based on keystroke dynamics.," Int. J. Inf. Sec., vol. 1, no. 2, pp. 69-83, 2002.

[22] F Bergadano, D Gunetti, and C Picardi, "User authentication through keystroke dynamics," ACM Transactions on Information and System Security, vol. 5, no. 4, Nov 2002

[23] BioPassword, "Biopassword-questions and answers," Tech report, BioNet Systems, LLC, 2002.

[24] George Shaffer, "Geodsoft good and bad passwords how-to: An example list of common and especially bad passwords," 2004, http://geodsoft.com/howto/password/common.htm.

[25] Volker Hosel, "Statistics in biosciences ii," Tech. Rep., Munich University of Technology, 2004.

[26] Lan Guo, Yan Ma, Bojan Cukic, and Harshinder Singh, "Robust prediction of fault-proneness by random forests," Nov 2004 .

[27] "Software package weka," 2005, http://www.cs.waikato.ac.nz/ml/weka/.

[28] "Software package c5.0 / see5," 2004, http://www.rulequest.com/see5-info.html. 
[29] "Software package r," 2005, http://www.r-project.org/.

[30] "R random forests add-in," 2005, http://www.maths.lth.se/help/R/.R/library/randomForest/html/randomForest. html.

[31] Leo Breiman, "Random forests," Machine Learning, vol. 45, no. 1, pp. 5-32, 2001.

[32] Leo Breiman and Adele Cutler, "Random forests," Technical report, Department of Statistics, University of California at Berkeley, Berkeley, CA, 1999, http://stat-www.berkeley.edu/users/breiman/RandomForests/cc_home.htmi.

[33] C G G Aitken, Statistics and the Evaluation of Evidence for Forensic Scientists, John Wiley \& Sons, NY, 1997.

[34] Yoav Freund and Robert E. Schapire, "Large margin classification using the perceptron algorithm," in COLT' 98: Proceedings of the eleventh annual conference on Computational learning theory, New York, NY, USA, 1998, pp. 209-217, ACM Press.

[35] Frank Rosenblatt, "The perceptron: A probabilistic model for information storage and organization in the brain," Psychological Review, vol. 65, no. 38, 1958.

[36] Wikipedia, "Neural networks," http://en.wikipedia.org/wiki/NeuralNetworks.

[37] J H Friedman, "Greedy function approximation: A gradient boosting machine," Technical report, Department of Statistics, Stanford University, 1999.

[38] RULEQUEST Research, "Data mining tools see5 and c5.0," Tech. Rep., RULEQUEST Research, Nov 2004.

[39] Enrique Langridge, "Student's t-test," http://www.cem.itesm.mx/profesores/dp/eleon/t-test.htm.

[40] Anil K. Jain and Arun Ross, "Learning user-specific parameters in a multibiometric system," in International Conference on Image Processing (ICIP), Rochester, NY, Sep 2002.

[41] Matt Bishop, Computer Security: Art and Science, Addison-Wesley, Boston, 2003.

[42] S. Salzberg, "On comparing classifiers: Pitfals to avoid and a recommended approach," Data Mining and Knowledge Discovery, vol. 1, no. 3, pp. 317-327, 1997.

[43] J. Ross Quinlan, "Induction of decision trees," Machine Learning, vol. 1, pp. 81 - $106,1986$.

[44] J. Ross Quinlan, C4.5: Programs for Machine Learning, Morgan Kaufmann, 1992.

[45] F. Rosenblatt, Principles of Neurodynamics: Perceptrons and the theory of brain mechanisms, Spartan Books, 1962.

[46] J. Ross Quinlan, "Decision trees and multivalued attributes," vol. 11, pp. $305-318,1988$. 